PAPERS IN PHYSICAL OCEANOGRAPHY AND METEOROLOGY Published by MASSACHUSETTS INSTITUTE OF TECHNOLOGY AND WOODS HOLE OCEANOGRAPHIC INSTITUTION (In continuation of Massachusetts Institute of Technology Meteorological Papers) VOL. III, NO. 3

\title{
THE LAYER OF FRICTIONAL INFLUENCE IN WIND AND OCEAN GURRENTS
}

\author{
BY \\ C.-G. ROSSBY and R. B. MONTGOMERY \\ Contribution No. 7I from the Woods Hole Oceanographic Institution
}

CAMBRIDGE, MASSACHUSETTS

April, 1935 


\section{CONTENTS}

I. INTRODUCtION

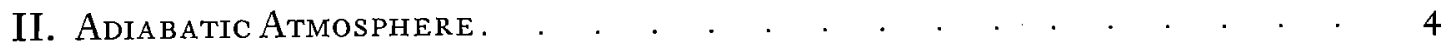

I. Completion of Solution for Adiabatic Atmosphere . . . . . . . . . 4

2. Light Winds and Residual Turbulence . . . . . . . . . . 22

3. A Study of the Homogeneous Layer at Boston . . . . . . . . 25

4. Second Approximation . . . . . . . . . . . . . . . . . . . . . 40

III. Influence of Stability

I. Review . . . . . . . . . . . . . . . . . . . . 44

2. Stability in the Boundary Layer . . . . . . . . . . . . 47

3. Stability within Entire Frictional Layer . . . . . . . . . . 56

IV. Application to Drift Currents . . . . . . . . . . . . . . . . . . 64

I. General Comments . . . . . . . . . . . . . . . . 64

2. The Homogeneous Layer . . . . . . . . . . . . . . 66

3. Analysis of Material . . . . . . . . . . . . . . . . . . 6767

4. Results of Analysis . . . . . . . . . . . . . . . . . . . . . . . . 69

5. Scattering of the Observations and Advection . . . . . . . . . $\quad$. 72

6. Oceanograph Observations .. . . . . . . . . . . . . . . . . . . 73

7. Wind Drift of the Ice . . . . . . . . . . . . . . . . . . .

V. Relation between the Velocity Profile and the Value of the Mixing LenGth . . . . . . . . . . . . . . . . . . . . 85

I. Theoretical Comments . . . . . . . . . . . . . . . . . 85

2. Stirring in Shallow Water . . . . . . . . . . . . . . 88

Appendix . . . . . . . . . . . . . . . . . . . . . 92

Modified Computation of the Boundary Layer in Drift Currents . . . . . 92

Summary . . . . . . . . . . . . . . . . . . . . . . . . 98

RefEREnCES . . . . . . . . . . . . . . . . . . . . . . . . 99 


\section{INTRODUCTION}

The purpose of the present paper is to analyze, in a reasonably comprehensive fashion, the principal factors controlling the mean state of turbulence and hence the mean velocity distribution in wind and ocean currents near the surface. The plan of the investigation is theoretical but efforts have been made to check each major step or result through an analysis of available measurements. The comparison of theory and observations is made difficult by the fact that in most cases measurements have been arranged without the aid of a working hypothesis concerning the dynamics of the effect studied; thus information is often lacking concerning parameters essential to the interpretation of the data.

A complete description of the effect of turbulence upon the mean motion is possible when we know, in each point of the fluid, the six components of Reynolds' symmetric stress tensor as functions of time. If, in addition, a complete description of the effect of turbulence on the distribution of various suspensions and solutes or upon the temperature distribution in the medium is desired another field tensor must be determined, the Austausch tensor. ${ }^{1 *}$

For many meteorological and oceanographical purposes it is sufficient to consider individual cases where the mean motion is stationary, horizontal and independent of the horizontal coordinates. In so far as its effects upon the mean motion are concerned, the state of turbulence in any given point may then be described with the aid of a single quantity, the eddy-viscosity coefficient, which, however, varies greatly from point to point along the vertical and from case to case. Making the further assumption that the horizontal gradients of the various extraneous fields under consideration (for example temperature and specific humidity in air, salinity in water) are negligibly small, one component only of the Austausch tensor need to be taken into consideration. This component is identical with Wilhelm Schmidt's Austausch, which may or may not have the same value as the eddy-viscosity coefficient. Knowing the Austausch coefficient in each point of the fluid we are in a position to determine the rate of vertical transfer of various field properties from their vertical gradients.

We know from numerous investigations in the last thirty years that the great variations of the eddy-viscosity are controlled by certain -external parameters; the most important of these are, in the case of the atmosphere, gradient wind velocity (horizontal pressure gradient), character (roughness) of the ground, vertical temperature gradient (stability), and latitude. In oceanic drift currents the corresponding factors are: surface wind velocity (determining the frictional drag and also the roughness of the sea surface), internal vertical stability, and latitude. Turbulence in bottom currents is, of course, influenced by the character of the ocean bottom. Furthermore, as in air, the recent history of the body of water under consideration may be of significance, i.e. the state of turbulence may not adjust itself instantaneously to the prevailing values of the controlling external parameters but may show some lag.

The present investigation aims at the development of a quantitative theory for the eddy-viscosity coefficient in terms of the controlling parameters listed above. The eddyviscosity is never measured directly but always obtained by indirect means from ob-

\footnotetext{
* References are indicated by superior figures and are listed at the end of the paper.
} 
served velocity distributions. Some of the earlier methods used in this calculation are entirely unsatisfactory or have been applied, incorrectly, to mean velocity distributions obtained from heterogeneous data, giving eddy-viscosity values which are entirely fictitious. However, even the best methods of computing the eddy-viscosity coefficient give values which may be correct only to the order of magnitude. For this reason it seems preferable to check the theoretical results by a study of the observed velocity distribution for different values of the controlling parameters rather than by a study of generally rather uncertain eddy-viscosity values obtained indirectly from the same velocity profiles.

In the development of a rational theory for the eddy-viscosity and its interaction with the mean motion, liberal use of a fairly complicated but inexact mathematical apparatus is unavoidable. The physical principles upon which the theoretical discussion is based cannot always be said to have a rigid foundation; they are largely intuitive. It is hardly appropriate to erect an exact mathematical structure upon a weak physical foundation.

Our immediate object must be to indicate the general character of the relationships connecting the mean motion and hence the eddy-viscosity with the controlling external parameters and to serve as a guide in the organization of the proper measurements and in their interpretation. Under these circumstances the use of intuitive methods becomes permissible.

The introduction of the non-dimensional Reynold's number has served as a guiding principle and brought order and clarity to the field of aerodynamics. In the study of turbulent wind and ocean currents no such guiding principle exists. The writers believe that the ideas suggested below eventually will tend to remove this serious difficulty.

The present investigation forms a continuation of certain studies published by the first named author in $1932 .^{2}$ Frequent use will be made of the results obtained in this paper; for the sake of brevity, it will be referred to under the symbol A. Thus A 163 refers to formula 163 in A.

\section{ADIABATIC ATMOSPHERE}

\section{i. Completion of Solution for Adiabatic Atmosphere}

Two theoretical problems were treated in A. The first referred to a permanent drift current maintained by a steady wind in the surface layer of an unlimited homogeneous ocean. The second concerned the wind distribution maintained in a homogeneous (adiabatic) atmosphere by a constant horizontal pressure gradient. These two problems were solved with the aid of von Kármán's theory for the mixing length. ${ }^{3}$ In each case it was found that the eddy-viscosity coefficient ( $\eta$, referred to as $A$ in A) decreases with increasing distance $(z)$ from the boundary according to the law $\eta \propto(h-z)^{2}$. The distance $h$ at which $\eta$ disappears depends in a definite fashion upon the given external parameters. The mixing length $(l)$ also decreases with increasing distance from the boundary and is proportional to $h-z$.

The corrugations of the ground and of the ocean surface must set an upper limit for the magnitude of the mixing length at the boundary and this upper limit must reduce to the dimensions of the molecular free path in the case of an absolutely smooth surface. It is obvious, therefore, that the solutions obtained in A and leading to increasing values of the mixing length with approach towards the boundary must be supplemented with solutions applicable in the immediate vicinity of the surface. 
The solution of the atmospheric problem treated in A was incomplete also in another respect; the angle between surface wind and gradient wind remained undetermined. The analogous oceanic problem, treating the modifications set up in a steady gradient (slope) current due to frictional drag at the bottom, is of fundamental importance in Ekman's current theory. ${ }^{4}$ In each case the angle between the surface (bottom) velocity vector and the gradient velocity vector controls the transversal circulation (net transport across the isobaric surfaces). In Ekman's theory the transversal component of the bottom current represents the sole agent through which convergence or divergence of surface water can be offset. Since the permanent atmospheric wind system is of such a character as to maintain pronounced convergence in certain ocean regions, divergence in others, the significance of this angle from the point of view of the theory of ocean currents becomes apparent.

The first step in our investigation will consist in an effort to supply the missing elements which have been referred to above. Later other parameters such as stability, will be considered.

In his paper on "Meteorologische Anwendung der Strömungslehre" Prandt $1^{5}$ has given a simple solution for the wind distribution in the vicinity of the ground, that is, in the region where the theory of A ceases to apply. Prandtl's solution may be summarized thus:

We consider a boundary layer $H$ which is so thin that its motion is controlled entirely by the frictional drag on its two horizontal boundaries, i.e. it is assumed that volume forces (horizontal pressure gradient, deflecting force) may be neglected. The frictional drag per unit horizontal area will then be constant in intensity and direction from the ground up to the level $H$. The wind direction must also be constant within the layer $H$ and must coincide with the direction of the frictional drag. The assumption that all volume forces may be neglected represents a severe restriction; its significance will be discussed in a later section (II, 4 ).

Within the layer $H$ Prandtl assumes the mixing length $(l)$ to vary directly with the distance $(z)$ from the boundary. At the ground, $l$ has a finite value which, as a first approximation, may-be assumed-to-be-proportional to the average height $(\epsilon)$ of the-roughness elements of the ground. The variation of $l$ within the layer $H$ may then be expressed through the relation

$$
l=k_{0}\left(z+z_{0}\right)
$$

where the non-dimensional constant $k_{0}$ according to Prandtl and von Kármán has the value 0.38 . The constant $z_{0}$ is, according to the statement above, proportional to $\epsilon$,

$$
z_{0}=s \epsilon .
$$

The value of the factor $s$ is not definitely known. It probably depends upon the shape and distribution of the roughness elements, but Prandtl suggests a tentative value for $s$ of about $\mathrm{I} / 3 \mathrm{O}$.

With these assumptions the expression for the frictional stress $(\tau)$ may be written in the form

$$
\tau=\rho l^{2}\left(\frac{d W}{d z}\right)^{2}=\rho k_{0}{ }^{2}\left(z+z_{0}\right)^{2}\left(\frac{d W}{d z}\right)^{2}
$$

where $\frac{d W}{d z}$ represents the shear of the mean motion and $\rho$ represents the density. In the 
particular case under consideration $\tau$ is constant $\left(\tau=\tau_{0}\right)$ and the density change with elevation negligible. The preceding equation may therefore be integrated and gives

$$
\frac{d W}{d z}=\frac{\mathrm{I}}{k_{0}\left(z+z_{0}\right)} \sqrt{\frac{\tau_{0}}{\rho}}, \quad W=\frac{\mathrm{I}}{k_{0}} \sqrt{\frac{\tau_{0}}{\rho}} \ln \frac{z+z_{0}}{z_{0}}
$$

Turning this formula around we obtain

$$
\tau_{0}=\rho \frac{k_{0}^{2} W^{2}}{\left(\ln \frac{z+z_{0}}{z_{0}}\right)^{2}} .
$$

From a comparison of Prandtl's expression for the stress (3) with the one commonly used in meteorology it is seen that the eddy-viscosity must be given by

$$
\eta=\rho l^{2}\left|\frac{d W}{d z}\right|
$$

In the present case, then,

$$
\eta=\rho k_{0}\left(z+z_{0}\right) \sqrt{\frac{\tau_{0}}{\rho}}=\rho k_{0}^{2}\left(z+z_{0}\right) \frac{W_{a}}{\ln \frac{z_{a}+z_{0}}{z_{0}}},
$$

where $W_{a}$ represents the wind velocity at the anemometer level $\left(z_{a}\right)$. This important result shows that in a homogeneous atmosphere and in the immediate vicinity of the ground, the eddy-viscosity is a linear function of the distance from the ground and, at a fixed elevation, is directly proportional to the wind-velocity. We shall now present some observational evidence in support of the theory outlined above.

Prandtl's formula (5) for the stress agrees with an empirical formula obtained by G. I. Taylor ${ }^{6}$ from a study of Dobson's pilot balloon data from Salisbury Plain. Taylor found

$$
\tau_{0}=\rho \gamma^{2} W_{a}^{2}{ }^{*}
$$

The two formulae agree if

$$
\gamma^{2}=\frac{k_{0}^{2}}{\left(\ln \frac{z_{a}+z_{0}}{z_{0}}\right)^{2}} .
$$

This formula shows how Taylor's coefficient $\gamma^{2}$ varies with the elevation of the anemometer and with the roughness of the ground. Taylor's values for $\gamma^{2}$ range between $22 \times 10^{-4}$ and $32 \times 10^{-4}, W_{a}$ being measured $30 \mathrm{~m}$. above the ground.

Mildner ${ }^{7}$ has recently published a few determinations of $\eta$ from hodographs of vertical wind distributions observed near Leipzig. The hodographs were constructed from individual pilot balloon runs or from means of homogeneous data, and the $\eta$-values were then computed by Solberg's method. ${ }^{8}$ From the mean of 28 balloon runs made during one October day Mildner obtains the following vertical variation of $\eta$.

\footnotetext{
* In A the symbol $\kappa$ was used instead of $\gamma^{2}$.
} 
TABLE 1

$\begin{array}{lrrrrrrr}\text { Height }(z) \text { in m. } & 80 & 135 & 190 & 240 & 295 & 405 & 460 \\ \eta \text { in c.g.s. units } & 125 & 270 & 310 & 500 & 246 & \text { II } & 710\end{array}$

The hodograph for the corresponding wind distribution indicates a velocity of 9.3 m.p.s. at an elevation of $35 \mathrm{~m}$. above the ground. It will be shown below that the appropriate value of the roughness parameter $z_{0}$ for a smooth lawn lies in the vicinity of $0.5 \mathrm{~cm}$. Mildner's data were collected at an airport for which this $z_{0}$-value would seem to be appropriate. The objection might be made that the eddy-viscosity (i.e. the stirring) depends upon the roughness along the trajectory of the air current as well as upon the roughness at the point of observation and that for this reason the value of $z_{0}$ should be chosen so as to be characteristic of the entire trajectory. To this objection two comments may be added: first, that we believe that the adjustment of the state of turbulence to the prevailing external parameters is fairly rapid,* and second, that the parameter $z_{0}$ occurs in the logarithmic part of expression (7) for $\eta$ due solely to the substitution of the wind velocity at anemometer level for the stress. There can hardly be any doubt that in the relation between stress and wind velocity near the ground the appropriate $z_{0}$-value is the one characteristic of the point and moment of observation.

Inserting the above values of $W_{a}$ and $z_{0}$ in (7) we obtain

$$
\eta=0.02\left(z+z_{0}\right)
$$

in excellent agreement with Mildner's values for the first $240 \mathrm{~m}$. above the ground. The rapid decrease in the observed $\eta$-values beyond $240 \mathrm{~m}$. indicate very plainly that the validity of the preceding theory is restricted to a layer in the vicinity of the ground. Even if we were to take into account the gradual decrease of the stress

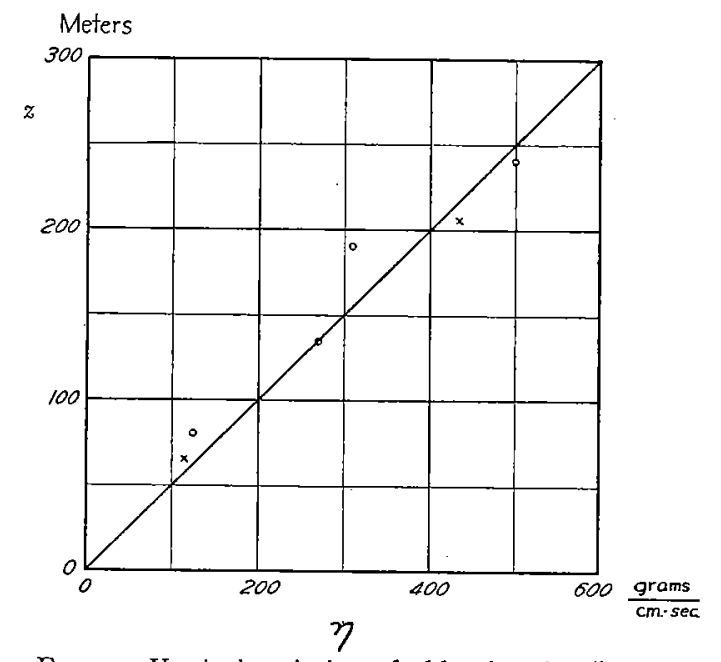

FIG. I.-Vertical variation of eddy-viscosity (based on Mildner's data).

with elevation by introducing a variable $\tau$ in (3), it would be found that the assumption of a mixing length proportional to the distance from the ground leads to impossible results beyond a certain elevation.

Mildner determined additional $\eta$-values from two individual balloon runs of the group discussed above. Of these the balloon run at o9I 5 gave quite low $\eta$-values, probably due to the stabilizing influence of remnants of an early morning temperature inversion. The pilot balloon run made at noon gave the following $\eta$-values:

TABLE 2

$\begin{array}{lrrrr}\text { Height }(z) \text { in m. } & 65 & 205 & 360 & 5 \text { IO } \\ \eta \text { in c.g.s. units } & \text { II } 5 & 435 & 225 & 50\end{array}$

The two first $\eta$-values of this table as well as the four first $\eta$-values of Table I are plotted in Fig. I together with the theoretical line (IO).

* Compare II, 3 . 
Mildner states that the lapse rate on the day of his observations was in the vicinity of $0.65^{\circ} \mathrm{C}$ per $100 \mathrm{~m}$. It will be seen below that the degree of stability indicated by this lapse rate is quite effective in suppressing turbulence. However, the air reaching the airport at which Mildner's observations were made had just before passed over the city of Leipzig. It seems fairly certain that the highly increased stirring resulting from the passage of the wind over a large city must have brought about complete mixing and the establishment of an adiabatic lapse rate in the lowest two hundred meters, as indicated by the surprisingly high level at which the maximum value of $\eta$ was found and the abnormally rapid decrease of $\eta$ above this level.

The ratio between the wind velocities at two levels $z_{1}$ and $z_{2}$ near the ground is given by

$$
\frac{W_{2}}{W_{1}}=\frac{\ln \frac{z_{2}+z_{0}}{z_{0}}}{\ln \frac{z_{1}+z_{0}}{z_{0}}}=\frac{\log \left(z_{2}+z_{0}\right)-\log z_{0}}{\log \left(z_{1}+z_{0}\right)-\log z_{0}}
$$

At all levels except those a few centimeters above the ground, $z_{0}$ is negligible compared with $z_{1}$ and $z_{2}$. Thus the above ratio reduces to the form

$$
\frac{W_{2}}{W_{1}}=\frac{\log z_{2}-\log z_{0}}{\log z_{1}-\log z_{0}}
$$

which is well suited for determinations of $z_{0}$. If anemometer readings are available from three levels we may form the ratio

$$
\frac{W_{3}-W_{2}}{W_{2}-W_{1}}=\frac{\log z_{3}-\log z_{2}}{\log z_{2}-\log z_{1}}
$$

which is independent of both stress and roughness parameter and depends solely upon the three anemometer levels. Equation (I 2 ) is particularly useful in attempts to verify the theory.

Hellmann's wind velocity measurements in Nauen and in Potsdam offer an excellent opportunity for a check of the preceding theoretical discussion and for a determination of $z_{0}$. Observations were collected for a period of about four years at three (later five) levels over a level grass-field or lawn northwest of the radio station in Nauen. In Hellmann's final report, ${ }^{9}$ no subdivision of the material according to wind velocity is made and for this reason the data are less suitable for our purposes. In an earlier paper, ${ }^{10}$ Hellmann presents a survey of preliminary results obtained from measurements during the period December IO, I9I 2 to November 20, I9I3. These data will be used in the following analysis.

Hellmann's anemometers were mounted at three levels, $2 \mathrm{~m}$., $16 \mathrm{~m}$., and $32 \mathrm{~m}$., above the ground. From the entire observational material for this first year, Hellmann selects two groups, referred to as days with light wind and days with strong wind. Days with light wind are defined by the condition that the 24 -hour mean of the wind velocity $2 \mathrm{~m}$. 
above the ground must be $\leq 2$ m.p.s. During days with strong wind the same mean must be $\geq 5$ m.p.s. Table 3 is a reproduction of Hellmann's Table 4 and gives ratios and differences between the wind readings at the three different levels for light and for strong wind. It is reasonable to assume that the lapse rate is practically dry-adiabatic between 0900 and 1500 on windy days. In Fig. 2 we have plotted Hellmann's mean

values of $\frac{W_{16}}{W_{2}}$ and $\frac{W_{32}}{W_{2}}\left(=\frac{W_{32}}{W_{16}} \cdot \frac{W_{16}}{W_{2}}\right)$ for the period $0900-1500$ for the entire year against $\log z$. Corresponding ratios for the summer half year and for the winter half year have been entered on the same diagram. The points fall very nearly on a straight line, the slope of which indicates a $z_{0}$-value of $0.55 \mathrm{~cm}$. Assuming Prandtl's value for $s(=\mathrm{I} / 3 \mathrm{O})$ to be correct, this corresponds to a roughness of about $16 \mathrm{~cm}$., which would seem to be a reasonable value for level, grass covered fields.

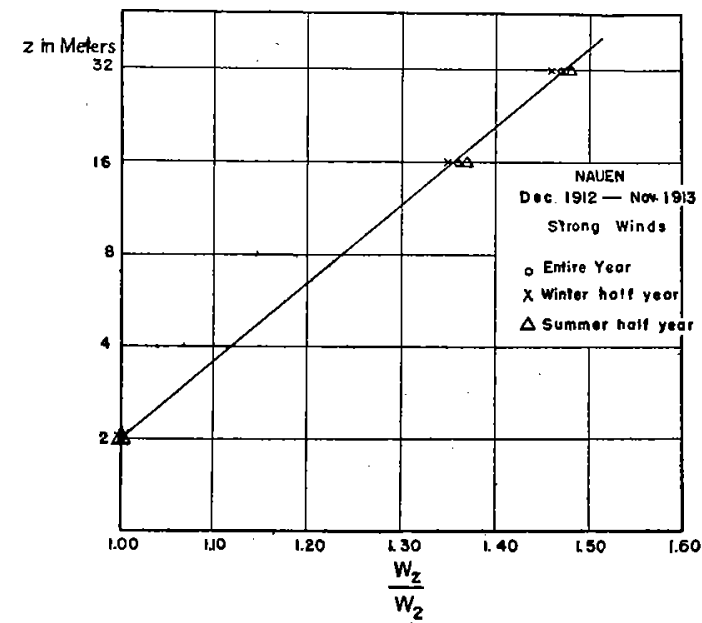

FIG. 2.- Vertical variation of strong winds with elevation (from Hellmann's data).

During the autumn of I9I 8 Hellmann ${ }^{11}$ measured the wind velocity at five different levels above a smooth lawn near Potsdam. The grass was cut repeatedly within the area $\left(100 \mathrm{m.}^{2}\right)$ where the anemometers were mounted, 5, 25, 50, 100 and $200 \mathrm{~cm}$. above the ground. The anemometers had a cup diameter of $4 \mathrm{~cm}$. In view of the strong wind gradient next to the ground and the relatively large diameter of the cups it is to be expected

TABLE 3

Ratios and Differences of Wind Velocities at Different Levels During Days with Light Wind and Days with Strong Wind (Hellmann).

$\mathrm{C}$ stands for winter half year, $\mathrm{W}$ for summer half year and $\mathrm{Y}$ for entire year.

\begin{tabular}{|c|c|c|c|c|c|c|c|c|c|c|c|c|c|}
\hline \multirow[b]{2}{*}{ Hour } & \multicolumn{3}{|c|}{$\mathrm{I} 6 \mathrm{~m} .: 2 \mathrm{~m}}$. & \multicolumn{3}{|c|}{$32 \mathrm{~m} .: 16 \mathrm{~m}$} & \multicolumn{3}{|c|}{$\mathrm{I} 6 \mathrm{~m} \cdot-2 \mathrm{~m}$} & \multicolumn{3}{|c|}{$32 \mathrm{~m} .-16 \mathrm{~m}$} & \\
\hline & C & $W$ & $Y$ & C & W & $Y$ & $\mathrm{C}$ & W & $\mathrm{Y}$ & $\mathrm{C}$ & $\mathrm{W}$ & $\mathrm{Y}$ & \\
\hline $0-3$ & 2.07 & $2 \cdot 3^{2}$ & 2.23 & I . 28 & I . 42 & I $\cdot 37$ & I. 44 & I. 27 & $\mathbf{I} \cdot 33$ & 0.78 & 0.94 & 0.88 & \\
\hline $3-6$ & 2.03 & 2.22 & 2.16 & $I \cdot 3 I$ & I. 48 & I . $4^{\mathrm{I}}$ & I. 15 & I. 05 & 1.09 & 0.70 & $0.9 \mathrm{I}$ & 0.84 & \\
\hline $6-9$ & I. 75 & I . 40 & I. 50 & $1 \cdot 30$ & I. I 5 & I. 20 & 1.04 & 0.63 & 0.76 & 0.63 & 0.33 & 0.46 & \\
\hline $9-12$ & I. 26 & I. 26 & I. 26 & i. 05 & I. 06 & I. .06 & 0.55 & 0.57 & 0.57 & $0 . I_{4}$ & 0.18 & 0.16 & Light Wind \\
\hline I $2-15$ & I. 26 & 1.27 & 1.27 & 1.06 & I. .07 & 1.07 & 0.55 & 0.69 & 0.64 & 0.15 & 0.22 & 0.20 & Light Wind \\
\hline I $5-18$ & I. 68 & 1.40 & I. 46 & I. I6 & I. I 2 & I.I 2 & $0.9 x$ & 0.87 & 0.89 & 0.36 & 0.37 & 0.35 & \\
\hline I $8-2 I$ & I. 95 & I. $9 \mathrm{I}$ & $I .9 I$ & I. 29 & I. 25 & 1.27 & I. 26 & I. 37 & I. 32 & 0.75 & 0.71 & 0.74 & \\
\hline $21-24$ & I.9I & 2.19 & 2.07 & $1 \cdot 30$ & I. 33 & I. $3^{2}$ & I. 26 & I. 56 & I. 45 & 0.80 & 0.94 & 0.89 & \\
\hline Mean & I. 73 & 1.75 & I. 73 & I. 22 & I. 24 & I. 23 & I. 02 & 1.00 & I.OI & 0.54 & 0.58 & 0.56 & \\
\hline $0-3$ & $x .45$ & I. 5 I & I . 47 & I. 12 & I. I 5 & I. I3 & 2.44 & 2.26 & $2 \cdot 3^{8}$ & 0.94 & 0.99 & 0.95 & \\
\hline $3-6$ & I. 43 & I. 46 & I. 44 & I. 12 & I. 12 & $x .12$ & 2.40 & 2.29 & 2.37 & 0.93 & 0.97 & 0.95 & \\
\hline $6-9$ & I . 4I & I. 38 & I. 40 & I . I I & 1.09 & I. IO & 2.45 & $2 \cdot 46$ & 2.43 & 0.93 & 0.83 & 0.88 & \\
\hline $9-12$ & $I \cdot 36$ & I. 37 & I. 36 & I.08 & I. .08 & 1.08 & 2.60 & 2.68 & 2.63 & 0.74 & 0.80 & 0.76 & \\
\hline $12-15$ & I. 34 & I. 37 & I. 36 & I. .08 & I . O8 & I. .08 & $2.6 \mathrm{I}$ & 2.73 & 2.66 & 0.77 & $0.8 \mathrm{I}$ & 0.78 & Strong $W_{1}$ nd \\
\hline $15-18$ & I .40 & I. 38 & I . 40 & I.09 & I. .08 & 1.09 & $2.4^{8}$ & 2.66 & 2.55 & 0.75 & $0.8 \mathrm{I}$ & 0.84 & \\
\hline $18-21$ & I. 45 & I .45 & $x .45$ & I. I2 & I . I 2 & 1. I 2 & 2.50 & $2.3^{8}$ & 2.47 & 0.97 & 0.93 & 0.96 & \\
\hline $2 I-24$ & I. $4^{6}$ & x. 49 & I .47 & I . I I & I. 13 & I . I I & 2.47 & $2.2 \mathrm{I}$ & 2.40 & 0.87 & $0.9 \mathrm{I}$ & 0.87 & \\
\hline Mean & I. $4 \mathrm{I}$ & I. 43 & $Y \cdot 4^{2}$ & I. IO & I. I I & I . IO & 2.49 & $2 \cdot 4^{6}$ & 2.48 & 0.86 & 0.88 & 0.87 & \\
\hline
\end{tabular}




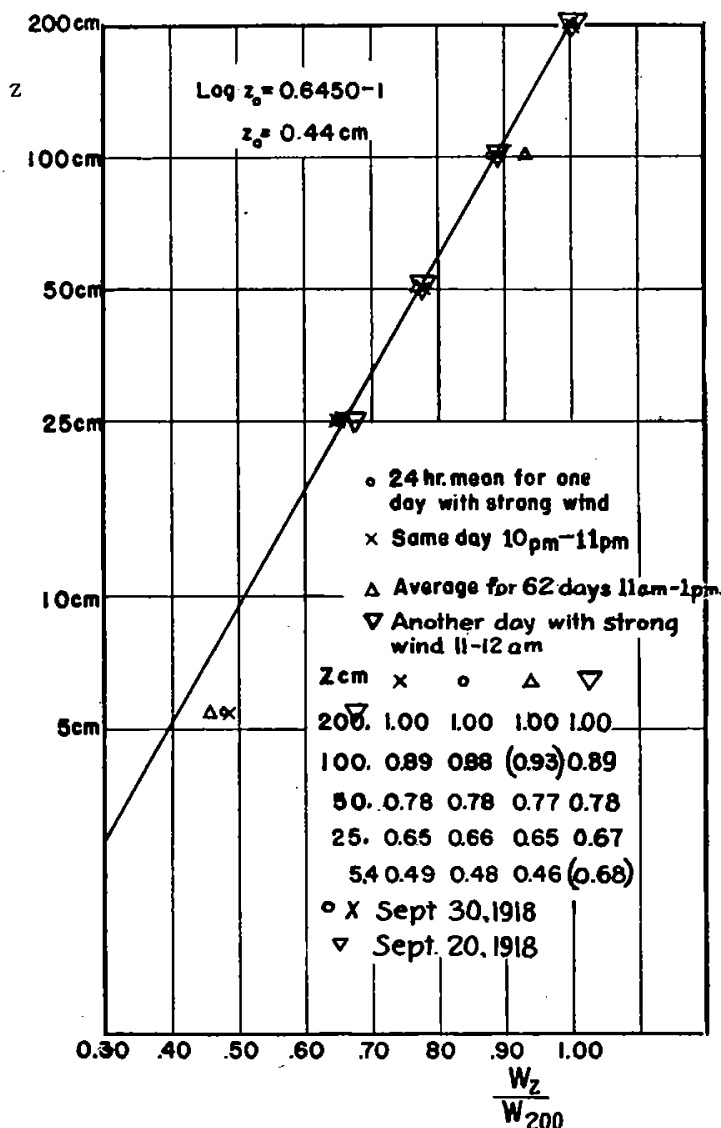

FIG. 3.- Vertical variation of wind velocity in the immediate vicinity of the ground (from Hellmann's data).

that the lowest anemometer should show too high wind velocities. Table I in Hellmann's paper ${ }^{11}$ gives the diurnal variation of the wind velocity at the different levels based on an observation period of 62 days. The table indicates fairly steady conditions between I IOO and I300. In the fall, in the middle of the day, the temperature lapse rate should be fairly close to the dry-adiabatic; thus the corresponding velocity distribution should be of the logarithmic type.

In Fig. 3 we have plotted the average values of the ratio $W_{z} / W_{200}$ for the middle of the day (I IO0-I300) against log $z$. The anemometer at $5 \mathrm{~cm}$. gives too high values for the wind ratio, the probable explana- tion of which has been mentioned above.* Also at $100 \mathrm{~cm}$. the ratio seems to be abnormally high. This may be due to some slight error in the calibration of this particular anemometer for the prevailing light winds (about 3.4 m.p.s.) since the discrepancy does not appear in some readings reproduced by Hellmann and entered in our figure to illustrate the vertical wind gradient on days with strong winds. The $z_{0}$-value indicated by this figure is $0.44 \mathrm{~cm}$., or somewhat less than the value obtained from Nauen.

Shaw ${ }^{12}$ has published average values for the ratio of the wind velocity at various levels between $0.5 \mathrm{~m}$. and $30 \mathrm{~m}$. to the wind velocity at $10 \mathrm{~m}$. These values, which are reproduced in Fig. 4, apply to open grass land. They are taken from "Annual Wind Summary I9I6" in "British Meteorological and Magnetic Year Book, I9I6." There is no information available concerning the time of day or the season to which these values apply. The slope of the line in Fig. 4 indicates a roughness parameter of 3.18 $\mathrm{cm}$., which would seem to be a reasonable

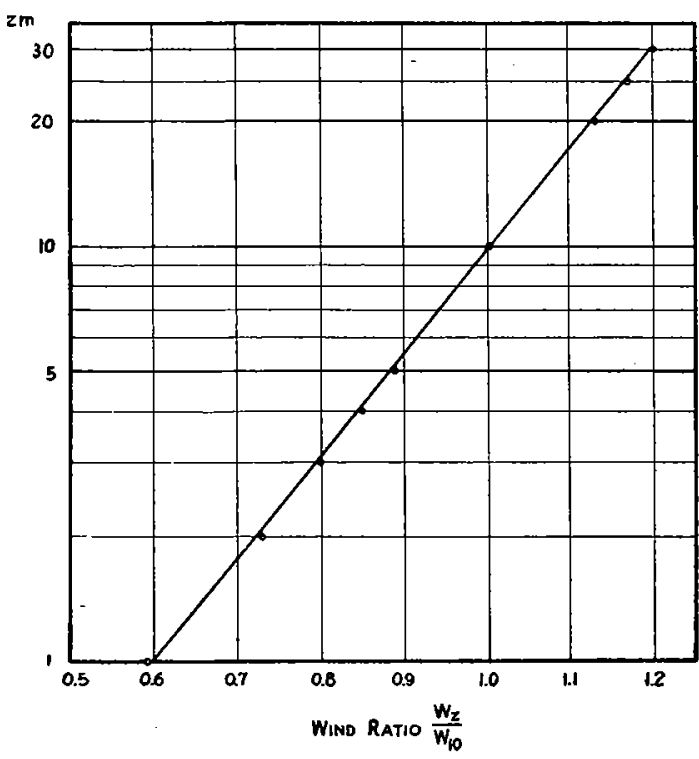

FIG. 4--Vertical variation of wind velocity over open grass land (from Shaw's data).

\footnotetext{
* At this lowest level it is necessary to use the exact relation (I Ia), so $W_{5} / W_{200}$ is plotted against $z+z_{0}=5.4 \mathrm{~cm}$.
} 
value for open grass land, broken by bushes, hedges and ditches, but it is also conceivable that the greater value of $z_{0}$ obtained from Shaw's ratios may be due to the inclusion of observations made in light winds under stable temperature conditions.

In September 1919, Wüst ${ }^{13}$ made some measurements of the vertical wind distribution at an anchor station in the southeastern part of the Baltic. The sea increased from I to 3 on the international scale during the 24 -hour period of the observations. Wüst gives some values for the vertical temperature gradient during approximately the same period (Wüst's Table 2I, I). They indicate a superadiabatic lapse rate between $0.2 \mathrm{~m}$. and I.2 $\mathrm{m}$. above sea level, but a slight inversion from $2 \mathrm{~m}$. to $6 \mathrm{~m}$. However, the air temperatures measured between $2 \mathrm{~m}$. and $6 \mathrm{~m}$. were, according to Wüst, influenced by radiation from the vessel, while the temperatures between $0.2 \mathrm{~m}$. and $\mathrm{i} .2 \mathrm{~m}$. were measured from a dory and should be reliable. From these comments it would appear that there was no marked inversion present to produce strong wind shear and thus lead to artificially high values of $z_{0}$.

Wüst's wind values are given in Table 4 . The graphical representation in Fig. 5 indicates a $z_{0}$-value of about $4 \mathrm{~cm}$., reasonable in view of the state of the sea during the observation period.

TABLE 4

$\begin{array}{cccc}\text { Height IN } m . & W \text { IN m.p.s. } & \frac{W}{W_{1}} & z_{0} \\ & & & \\ 6 & 5.24 & \mathrm{I} .56 & 3.98 \\ 2.5 & 4.32 & \mathrm{I} .28 & 3.89 \\ \mathrm{I} .0 & 3.37 & 1.00 & - \\ 0.2 & 2.84 & 0.84 & (0.00)^{*}\end{array}$

The wind velocity at $0.2 \mathrm{~m}$. seems very

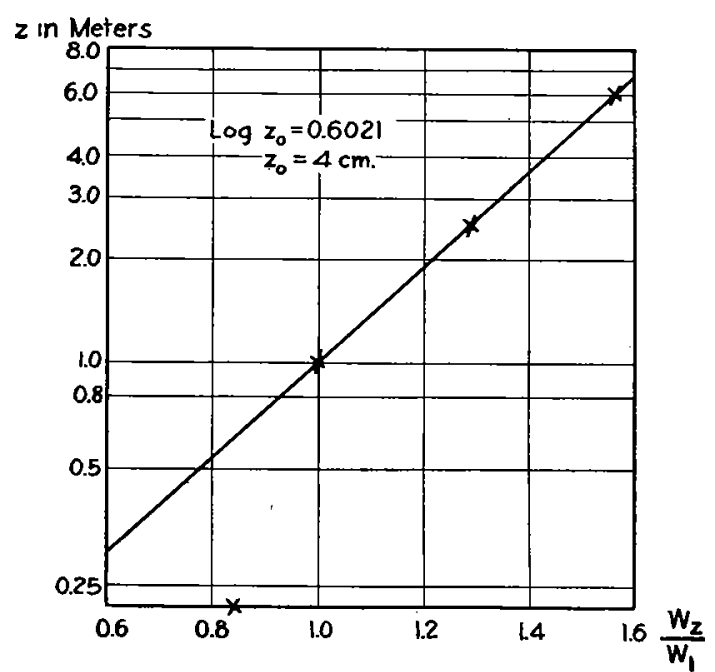

FIG. 5.-Vertical variation of the wind velocity above the sea surface (from Wüst's data). high. Wüst interprets this value as indicating slipping at the surface. It is probable that the strong wind gradient next to the surface may have contributed to the abnormally high value recorded by the anemometer.

The preceding discussion and illustrations should suffice to prove that the normal velocity distribution next to the surface is of the logarithmic type predicted by Prandtl's theory. Logarithmic formulae for the wind distribution near the ground are not new to meteorology; formulae of the type

$$
W=a \log (z+b)+c
$$

have been suggested by Hellmann ${ }^{9}$ and Chapman. ${ }^{14}$ However, this as well as other empirical formulae have had no theoretical foundation and their various constants have never been interpreted in terms of the significant physical parameters.

Establishing the validity of the logarithmic velocity distribution is by no means identical with proving the validity of Prandtl's entire theory. To do the latter we must verify the relation (5) between stress and wind velocity. $\dagger$ This is easily done for flow

* This point should have been plotted as indicated in the footnote on page 10, i.e. at the ordinate 0.24 .

$\dagger$ The $\eta$-values determined by Mildner and plotted in our Fig. I furnish such a verification. 
through tubes, where $\tau$ follows directly from the pressure gradient along the axis of the tube and thus may be regarded as given. On the other hand, to determine the frictional drag between the free atmosphere and the ground we must know the wind distribution within the entire frictional layer.

As a preliminary check we may calculate the coefficient $\gamma^{2}$ from (9) to see if the values so obtained agree with Taylor's empirical values. It was found, with the aid of Hellmann's observations, that a $z_{0}-\mathrm{v}$ alue of $0.5 \mathrm{~cm}$. is fairly characteristic of a smooth grass field or lawn. If this value is inserted in (9) and if we assume a $z_{a}$-value of $30 \mathrm{~m}$. in accordance with the conditions characteristic of Taylor's data, it follows that

$$
\gamma^{2}=19 \cdot 10^{-4}
$$

This value is just below the lower limit of the range in $\kappa^{2}$ obtained by Taylor. If we insert the value of $z_{0}$ characteristic of open grass land, $3.2 \mathrm{~cm}$., the result is

$$
\gamma^{2}=3 \mathrm{I} \cdot \mathrm{IO}^{-4}
$$

close to the upper limit of 'Taylor's range.

Assuming a constant gradient wind $U_{g}$ along the $x$-axis, the horizontal equations of steady motion may be written in the form

$$
-\rho f i\left(U+i V-U_{\vartheta}\right)+\frac{\partial}{\partial z}\left(\tau_{x}+i \tau_{y}\right)=0,
$$

where $U$ and $V$ represent the two mean wind velocity components, $\tau_{x}$ and $\tau_{y}$ the components of stress, and $\rho$ the density.

$$
f=2 \Omega \sin L
$$

is the Coriolis' parameter, with the dimension of an angular velocity. $\Omega$ is the angular velocity of the earth around its axis and $L$ the latitude.

If we consider the real part of $\left(\mathrm{I}_{4}\right)$ it follows from an integration between the ground and the level $h$ that

$$
\left[\tau_{x}\right]_{0}^{h}=-f \int_{0}^{h} \rho V d z=-\rho f \int_{0}^{h} V d z .
$$

We choose for $h$ a level where constant gradient wind prevails. Then

$$
\tau_{x 0}=\rho f \int_{0}^{h} V d z
$$

Next to the ground, stress and wind must be parallel. Thus, if $\varphi_{s}$ represents the angle between the surface wind and the isobars,

$$
\tau_{x 0}=\tau_{0} \cos \varphi_{s},
$$

and

$$
\tau_{0}=\frac{\rho f}{\cos \varphi_{s}} \int_{0}^{h} V d z
$$


This formula is independent of any assumptions regarding the variation of $\eta$ or $\tau$ with elevation and may confidently be used to calculate $\tau_{0}$ whenever the gradient wind direction remains constant with elevation. Numerical examples of this method are found in $(\mathrm{IV}, 7)$.

Under certain conditions the determination of $\tau_{0}$ from (19) may be simplified by the following procedure, which, to our knowledge, is new.

The vertical distribution of the wind is normally obtained with the aid of pilot balloons ascending at a constant rate. The displacement of the balloon normal to the isobars during the time interval $t$ is

$$
D=\int_{0}^{t} V d t
$$

or, because of the constant rate of ascent $(C)$,

$$
D=\frac{I}{C} \int_{0}^{z t} V d z
$$

When the balloon reaches the gradient wind region it ceases to move normal to the isobars. Thus, if $D$ now indicates the total displacement of the balloon normal to the isobars, we have

$$
C D=\int_{0}^{h} V d z
$$

and consequently

$$
\tau_{0}=\frac{\rho f C D}{\cos \varphi_{s}}
$$

The ascensional rate $C$ is known and the distance $D$ may be taken directly from the graphical representation of the horizontal trajectory of the balloon without evaluation of the wind velocities and wind directions. To do so it is necessary to determine the asymptote to the trajectory representing movement of the balloon along the isobars. The length of the perpendicular from the starting point to this line gives $D$. Unfortunately, it is often difficult to determine accurately the position of the asymptote.

Under the auspices of the Deutsche Seewarte in Hamburg a number of pilot balloon observations have been made in various parts of the Atlantic Ocean and the results published in Aus dem Archiv der Deutschen Seewarte. ${ }^{15}$ In most cases these reports contain reproductions of the horizontal trajectories of the individual ascents. On each trajectory, marks indicate the successive positions of the balloon at the $1000 \mathrm{~m}$. level, the $2000 \mathrm{~m}$. level et cetera. The ascensional rate varied from one balloon run to the next but in each case the scale of the reproduction of the trajectory was chosen in such a fashion as to give a uniform velocity scale; thus, from the distance between two successive points on any balloon trajectory the mean horizontal velocity for the corresponding layer may be determined with the aid of a single velocity scale.

In view of the fact that the ascensional rate $(C)$ is unknown we must modify (2Ia) so as to eliminate $C$. If we indicate by $t_{0}$ the time required for the balloon to rise I000 $\mathrm{m}$., the expression for the stress may be written 


$$
\tau_{0}=\frac{\rho f}{\cos \varphi_{s}} \cdot C \cdot t_{0} \cdot \frac{D}{t_{0}}=10^{5} \cdot \frac{\rho f}{\cos \varphi_{s}} \cdot \frac{D}{t_{0}} .
$$

$D / t_{0}$ has the dimensions of a velocity and may be determined by setting off the length of the perpendicular from the starting point to the asymptote on the velocity scale.

It is easily seen that an error of $\alpha$ percent in the assumed ascensional rate will produce an error in $\tau_{0}$ as computed from (2Ia) or (2I $\mathrm{Ib}$ of $\alpha^{2}+2 \alpha$ percent. It is therefore advisable to correct the values of $C$ given by the ordinary formulae so as to include the effect of turbulence on the ascensional rate in the surface layer or else to restrict the application of the method outlined to trajectories obtained with the aid of two theodolites.

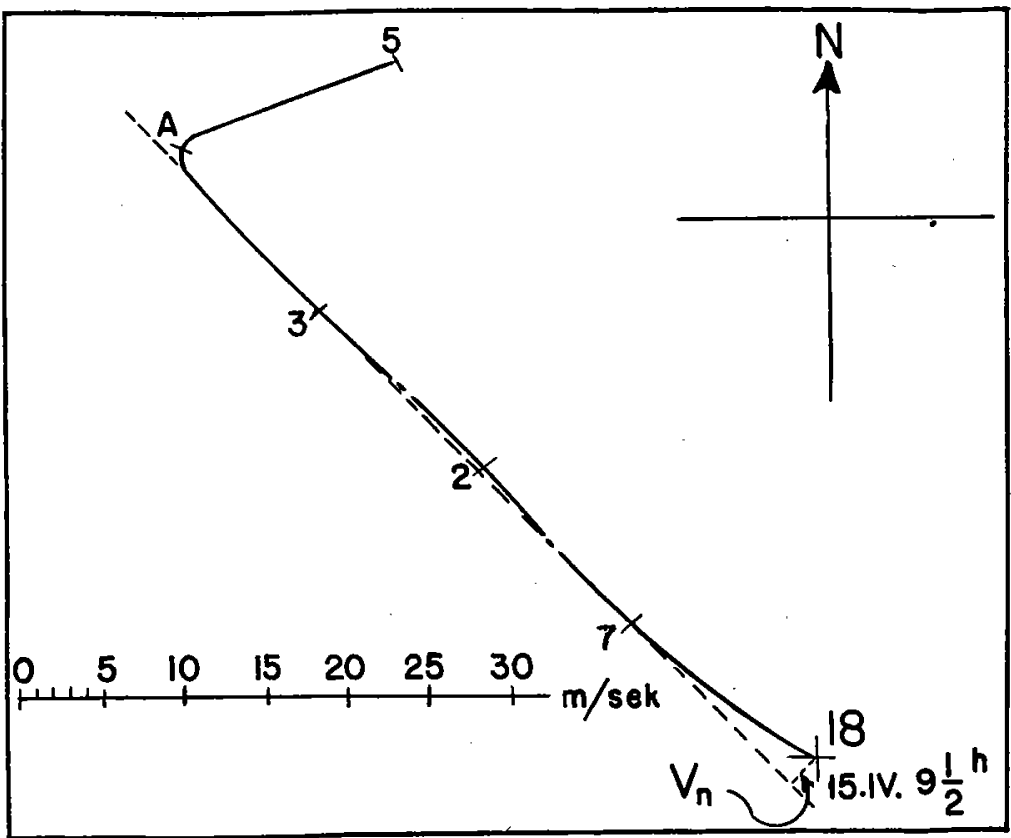

FIG. 6.-Determination of the surface wind force from pilot balloon trajectory. The trajectory is taken from "Aus dem Archiv der Deutschen Seewarte," Band

$$
46, \text { No. } 2 .
$$

Fig. 6 is an illustration of the method suggested above for the determination of the surface stress. The broken line represents the gradient wind path of the balloon. The short line marked $V_{n}$ represents the total displacement of the balloon normal to the isobars, here expressed as a velocity.

Averaging the results of such stress determinations from I 3 selected pilot balloon trajectories published in "Aus dem Archiv der Deutschen Seewarte," we obtain

$$
\frac{\tau_{0}}{p W_{a}^{2}}=\gamma^{2}=0.0013
$$

This value is unexpectedly low. In view of the difficulties associated with pilot balloon observations on board a moving steamer, too much weight should not be attached to this determination. 
The velocity distribution discussed in the preceding pages applies to a boundary layer $(H)$ within which the stress varies but little with elevation. The theory does not give us any information concerning the thickness of the boundary layer nor does it tell us the value of the stress. To determine these two quantities we must combine Prandtl's solution for the boundary layer with the solution derived in A for the free atmosphere. The latter theory was based upon the assumption that the atmosphere is subject to a vertically and horizontally constant pressure gradient and to a certain frictional drag parallel to the wind at the lower boundary, which we now take as the top of the boundary layer $H$. Measuring the vertical coordinate $z$ from this level, the theoretical solution in A may be summarized in the formulae below.

The mixing length is given by

$$
l=\frac{k(h-z)}{\sqrt{2}}
$$

where $z=h$ represents the level at which the frictional influence disappears and the actual wind becomes identical with the gradient wind. $h$ then is the thickness of the layer to which the solution in A applies. The non-dimensional constant $k$ was estimated to have a value of 0.065

If $\tau_{0}$ represents the intensity of the frictional drag at the lower boundary of the free atmosphere, then $h$ and $\tau_{0}$ are connected by the relation

$$
\tau_{0}=\rho \frac{f^{2} h^{2}}{9 k^{2}}
$$

which follows from a combination of (A I67) and (A I68).

The wind velocity at the lower boundary is

$$
W_{H}=U_{\theta}\left(\cos \varphi_{s}-\frac{\mathrm{I}}{\sqrt{2}} \sin \varphi_{s}\right)
$$

where $\varphi_{s}$ is the angle between the wind direction at the lower boundary (or at the ground) and the gradient wind direction. Between $\varphi_{s}$ and $h$ there exists a relation of the form

$$
h=\frac{9 k^{2}}{2} \frac{U_{g}}{f} \sin \varphi_{s}
$$

The wind distribution is characterized by a constant rate of shear $K$,

$$
K=\left|\frac{d U}{d z}+i \frac{d V}{d z}\right|=\frac{\sqrt{2} f}{3 k^{2}}
$$

If we indicate by $\psi$ the direction of the shearing vector, so that

$$
\frac{d U}{d z}+i \frac{d V}{d z}=K e^{i \psi}
$$


then

$$
\text { (A I44) } \quad z=h\left(\mathrm{I}-e^{\frac{\psi-\psi_{H}}{\sqrt{2}}}\right) \quad\left(\psi_{H}=\varphi_{s}\right)
$$

and the wind vector at any level is given by

$$
\mathrm{I}-\frac{U+i V}{U_{g}}=\left(\mathrm{I}-\frac{W_{H}}{U_{\theta}} e^{i \varphi_{s}}\right) e^{\left(i+\frac{1}{\sqrt{2}}\right)\left(\psi-\psi_{H}\right)}
$$

or

$$
\mathrm{I}-\frac{U+i V}{U_{g}}=\left(\mathrm{I}-\frac{W_{H}}{U_{g}} e^{i \varphi_{g}}\right) \cdot\left(\frac{h-z}{h}\right)^{1+i \sqrt{2} \tilde{2}} .
$$

With the aid of (A I66) this may be written

$$
\frac{U-U_{g}+i V}{U_{g}}=\sqrt{\frac{3}{2}} \sin \varphi_{s} \cdot e^{i\left(\pi-\beta+\varphi_{s}\right)} \cdot e^{\left(i+\frac{1}{\sqrt{2}}\right)\left(\psi-\psi_{H}\right)},
$$

From (6) it is seen that the eddy-viscosity is given by

$$
\left(\tan \beta=\sqrt{2}, \beta=54^{\circ} 44^{\prime}\right) \text {. }
$$

$$
\eta=\rho l^{2} K=\frac{\rho f}{3 \sqrt{2}}(h-z)^{2}
$$

and hence

(A 180 )

$$
\eta_{\max }=\frac{\rho f h^{2}}{3 \sqrt{2}}=\frac{27 k^{4}}{4 \sqrt{2}} \cdot \frac{\rho U_{o}^{2}}{f} \sin ^{2} \varphi_{s} .
$$

In patching together the two solutions we shall require continuity in frictional drag, in mixing length, and in wind velocity and wind direction. These conditions ensure continuity in eddy-viscosity and in rate of shear. Denoting by $H$ the thickness of the boundary layer within which Prandtl's solution obtains, the condition of continuity in the mixing length takes the form

$$
k_{0}\left(H+z_{0}\right)=\frac{k h}{\sqrt{2}}
$$

or, in view of the smallness of $z_{0}$,

$$
H=0 . \mathrm{I} 2 h \text {. }
$$

Since maximum eddy-viscosity occurs at the level $H,(26)$ indicates that in a turbulent layer of a total depth of $1000 \mathrm{~m}$., maximum stirring is reached $107 \mathrm{~m}$. above the ground. Combining (A 167 ) with (26) one finds that

$$
H=0.54 k^{2} \frac{U_{\theta}}{f} \sin \varphi_{s}
$$

showing that the level of maximum turbulence is displaced upward with increasing gradient wind and with decreasing latitude. 
From the required continuity in wind velocity and wind direction it follows, through combination of (4) and (A 166), that

$$
\frac{\mathrm{I}}{k_{0}} \sqrt{\frac{\tau_{0}}{\rho}} \ln \frac{H+z_{0}}{z_{0}}=U_{g}\left(\cos \varphi_{s}-\frac{\mathrm{I}}{\sqrt{2}} \sin \varphi_{s}\right),
$$

and, because of the continuity in frictional drag, substitution of (22) gives

$$
\frac{f h}{3 k k_{0}} \ln \frac{H+z_{0}}{z_{0}}=U_{v}\left(\cos \varphi_{s}-\frac{\mathrm{I}}{\sqrt{2}} \sin \varphi_{s}\right)
$$

In this formula $h$ may be eliminated by means of $(\mathrm{A}+67)$. The result is

$$
\frac{3 k}{2 k_{0}} \ln \frac{H+z_{0}}{z_{0}}=\cot \varphi_{s}-\frac{\mathrm{I}}{\sqrt{2}}
$$

and finally

$$
\frac{H+z_{0}}{z_{0}}=e^{\frac{2 k_{0}}{3 k}\left(\cot \varphi_{s}-\frac{1}{\sqrt{2}}\right)} .
$$

With the aid of (25) and (A 167 ) this formula reduces to

$$
N=\frac{U_{g}}{f z_{0}}=\frac{2 \sqrt{2} k_{0}}{9 k^{3}} \cdot \frac{\mathrm{I}}{\sin \varphi_{s}} \cdot e^{\frac{2 k_{0}}{3 k}}\left(\cot \varphi_{s}-\frac{1}{\sqrt{2}}\right) .
$$

Equation (3ra) provides us with an opportunity to calculate $\varphi_{s}$ from the nondimensional number $N$, which depends upon external parameters only, namely gradient wind speed, latitude and roughness of the ground. Presumably the same relation may be used to calculate the angle between bottom current and gradient current from the roughness of the bottom, the intensity of the bottom current and the latitude. Inserting the proper numerical values in ( $3 \mathrm{ra}$ ) we find

$$
N=\frac{U_{g}}{f z_{0}}=\frac{435}{\sin \varphi_{s}} e^{3.9 \cot \varphi_{s}-2.76}
$$

or

$$
\log N=\mathrm{I} .694 \cot \varphi_{s}-\log \sin \varphi_{s}+\mathrm{I} .44^{\mathrm{I}} \text {. }
$$

This equation is represented graphically in Fig. 7 by the curve marked $u=I$. The angle between the surface wind and the gradient wind increases with roughness and with latitude; it decreases with increasing gradient wind. However, the variations in $\varphi_{s}$, especially with the last two parameters, are quite limited. To illustrate this point Table 5 was prepared in which $N$ and $\varphi_{s}$ are tabulated as functions of latitude and gradient wind velocity for $z_{0}=3.18 \mathrm{~cm}$., the value characteristic of open grass land. The table indicates that a range in latitude of $70^{\circ}\left(20^{\circ}-90^{\circ}\right)$ produces a practically negligible variation $\left(2^{\circ}\right)$ in $\varphi_{s}$. For a given latitude, a range in $U_{g}$ of from 5 m.p.s. to $4 \circ \mathrm{m}$.p.s. corresponds to a range of $3^{\circ}$ in $\varphi_{s}$. 
PAPERS IN PHYSICAL OCEANOGRAPHY AND METEOROLOGY

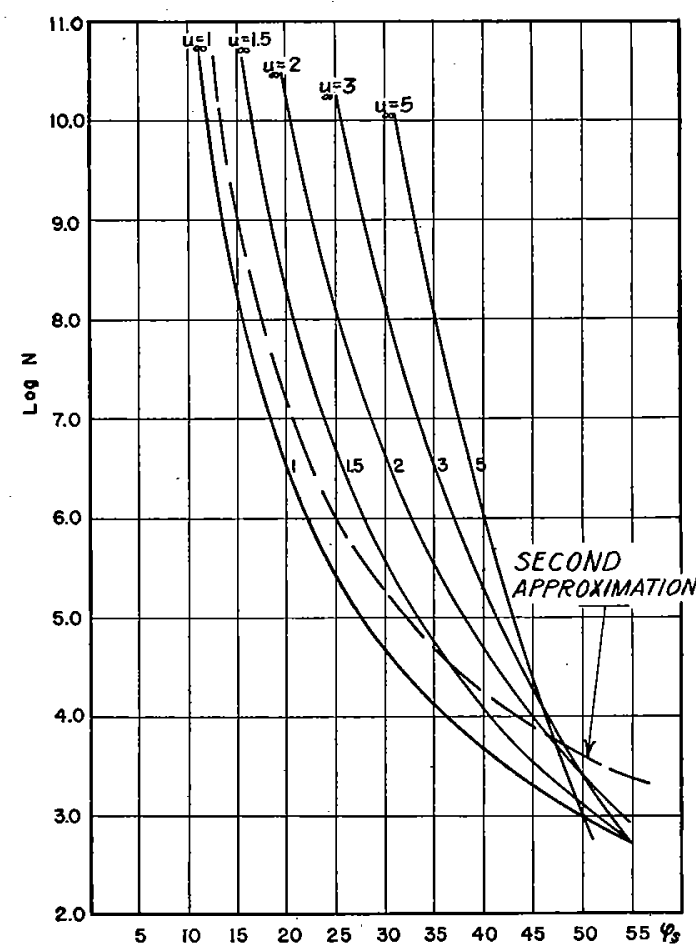

FIG. 7.-Relation between surface wind direction $\left(\varphi_{s}\right)$ and the non-dimensional number $N\left(=U_{o} / f z_{0}\right)$.
TABLE 5

\begin{tabular}{|c|c|c|c|c|}
\hline$L$ & $U_{g}=5$ m.p.s. & $U_{g}=\mathrm{IO}$ & $U_{G}=20$ & $U_{0}=4 \circ$ \\
\hline $20^{\circ}$ & $\begin{array}{l}N=3.2 \cdot 10^{6} \\
\varphi_{s}=20^{\circ}\end{array}$ & ${ }_{19^{\circ}}^{6.3 \cdot 10^{6}}$ & $\begin{array}{l}\text { I } 2.6 \cdot 10^{6} \\
18^{\circ}\end{array}$ & $\begin{array}{l}25.2 \cdot 10^{6} \\
17^{\circ}\end{array}$ \\
\hline 30 & ${ }_{21}^{2 .} .^{2}$ & $20^{4} \cdot 3$ & $\begin{array}{l}8.6 \\
19^{\circ}\end{array}$ & $\begin{array}{l}17.2 \\
18^{\circ}\end{array}$ \\
\hline 40 & ${ }_{2 \mathrm{I}}^{\mathrm{I}} \cdot \mathrm{o}^{7}$ & ${ }_{20}^{3} \cdot 3$ & $\begin{array}{l}6 \cdot 7 \\
19^{\circ}\end{array}$ & $\begin{array}{l}13.4 \\
18^{\circ}\end{array}$ \\
\hline 50 & $22^{1} \cdot 4$ & $\begin{array}{l}2.8 \\
21^{\circ}\end{array}$ & $20^{5.6}$ & $\begin{array}{l}\text { XI }:^{2} \\
\text { I } 8^{\circ}\end{array}$ \\
\hline 60 & $22^{\mathrm{I}} \cdot 3$ & $\begin{array}{r}2 \cdot 5 \\
21^{\circ}\end{array}$ & $20^{5} \cdot 0^{\circ}$ & $\begin{array}{l}10.0 \\
19^{\circ}\end{array}$ \\
\hline 70 & $22^{\mathrm{I}} \cdot{ }^{2}$ & $\underset{21^{2}}{2 \cdot 3}$ & $20^{4} \cdot 6$ & ${ }_{19}^{9.2}$ \\
\hline 80 & $\underset{22^{\circ}}{\mathrm{I}} \cdot \mathrm{I}$ & $21^{2} \cdot{ }^{2}$ & $20^{4} \cdot 4$ & $\begin{array}{l}8 \cdot 7 \\
19^{\circ}\end{array}$ \\
\hline 9 & ${ }_{22^{\circ}}{ }^{\circ}$ & $21^{2.2}$ & $20^{\circ} \cdot 3$ & $\begin{array}{l}8.6 \\
19^{\circ}\end{array}$ \\
\hline
\end{tabular}

It is seen that the effect of latitude on $\varphi_{s}$ is negligible for latitudes greater than $30^{\circ}$, the range being only $\mathrm{I}^{\circ} . \varphi_{s}$ depends primarily on $z_{0}$ and secondarily on $U_{g}$, so for all practical purposes, except at low latitudes, it can be readily found from Fig. 8. This figure was computed from the relation $N=U_{o} / f z_{0}$ and Fig. 7, using a middle-latitude value of $f=10^{-4}$, corresponding to $L=43^{\circ} 27^{\prime}$.

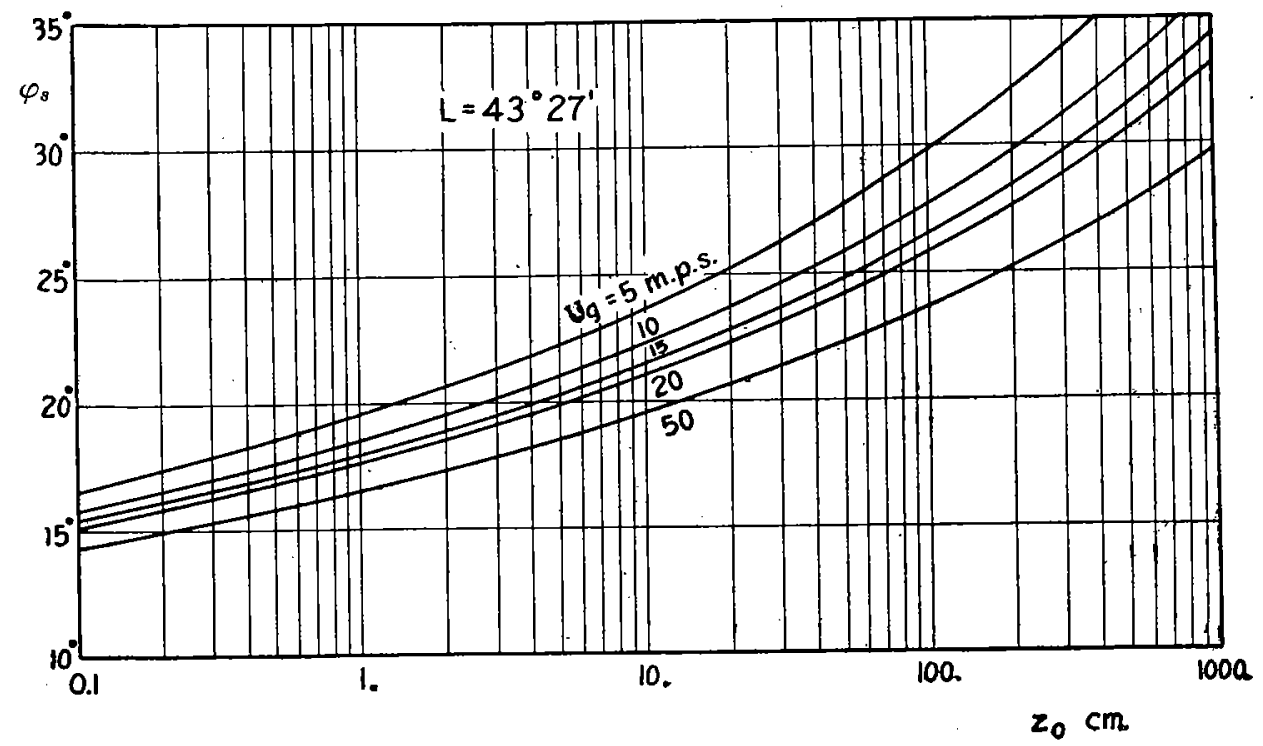

FIG. 8.-Relation between surface wind direction $\left(\varphi_{s}\right)$ and roughness $\left(z_{0}\right)$. 
In discussing the fundamental equation (3Ic) it is important to keep in mind that it applies to an adiabatic (but not unstable) atmosphere and that it assumes gradient wind direction and gradient wind velocity to be constant with elevation. If the gradient wind varies along the vertical, shearing forces must exist at higher levels which are independent of the frictional layer next to the ground; under those conditions the actual wind may nowhere coincide with the gradient wind and the preceding solution evidently does not apply. The ideal frictional layer is found in initially slightly stable air currents which, under the influence of moderate to strong, steady gradient winds, gradually are stirred to a height corresponding approximately to the theoretical limit $H+h$. If the initial stability is very pronounced the stirring may be insufficient to establish an adiabatic lapse rate all the way up to $H+h$; if the atmosphere initially is in superadiabatic equilibrium, or if the condensation level is lower than $H+h$, convection currents will develop. In either case the final régime will differ from the one here analyzed.

Keeping these restrictions in mind it is easily seen from dimensional considerations that $\varphi_{s}$, which is a pure number, must be a function of $U_{o} / f z_{0}$, this being the only nondimensional quantity which can be formed from the three external parameters $U_{o} f$ and $z_{0}$. It is well established that $\varphi_{s}$ increases with increasing roughness $\left(z_{0}\right)$. It follows that $\varphi_{s}$ must increase with $f$ and decrease with $U_{a}$.

Ekman ${ }^{16}$ has analyzed Jeffreys' data on the wind variations over the North Sea ${ }^{17}$ and states that there is no reliable correlation between $\varphi_{s}$ and $U_{\theta}$, a result which is in good agreement with the theoretical data in table 5. Jeffreys' data are divided in four groups, representing different intensities of the gradient wind. The corresponding mean values of $\varphi_{s}$ are given in Table 6.

\begin{tabular}{|c|c|c|c|c|}
\hline $\begin{array}{l}U_{a} \text { in m.p.s. } \\
\text { Number of observations } \\
\varphi_{s}\end{array}$ & $\begin{array}{l}\circ-8 \\
166 \\
15.7^{\circ}\end{array}$ & $\begin{array}{l}8.5^{-12} \\
164 \\
18.2^{\circ}\end{array}$ & 160 & $\begin{array}{l}>18 \\
71\end{array}$ \\
\hline
\end{tabular}

In view of the fact that the data on which this table is based* are given in compass points $\left(32\right.$ points $\left.=360^{\circ}\right)$, the numerical values of $\varphi_{s}$ for the different groups may not be very accurate. It is nevertheless interesting to note that $\varphi_{s}$ decreases slightly as $U_{g}$ increases from moderate to strong winds, in good agreement with our theoretical conclusions. For very low values of $U_{\theta}, \varphi_{s}$ is smaller. However, there is less reason to assume that the lapse rate is adiabatic and the gradient wind constant with elevation when $\mathrm{U}_{g}$ is small and the stirring slight. This point will be analyzed more closely in the next section. It is entirely reasonable to attribute most importance to the groups with moderate or strong winds in attempting to verify the theory.

Dobson's pilot balloon data, ${ }^{18}$ which were used by Taylor to verify his theory of the turbulent layer, show a very similar trend. They are reproduced in Table 7.

$\begin{array}{lccc}U_{o} \text { in m.p.s. } & \text { TABLE } 7 & \\ \text { Number of observations } & 4.6 & 9.1 & \text { I } 5.6 \\ \varphi_{\bullet} & .16 & 58 & 23 \\ \mathrm{~W}_{30 \mathrm{~m} .} / U_{0} & 13 . \mathrm{I}^{\circ} & 0.5^{\circ} & 19 \cdot 7^{\circ} \\ & 0.7^{\circ} & 0.65 & 0.6 \mathrm{I}\end{array}$

Assuming $z_{0}$-values of between $3.2 \mathrm{~cm}$. and $4 \mathrm{~cm}$., corresponding to Shaw's data for open grass land and to Wüst's data for the Baltic, and assuming $f$-values of $1.12 \cdot \mathrm{IO}^{-4}$

* Jeffrey's tables I and II. 
to I.I9. IO ${ }^{-4}$ we find, for $U_{g}=$ Io m.p.s., $N$-values in the vicinity of $5 \cdot 10^{6}$ and thus $\varphi_{\mathrm{s}}=19^{\circ}$, in good agreement with the observations.

It is more difficult to verify the variation of $\varphi_{s}$ with latitude. Ekman, in the paper already referred to, ${ }^{16}$ is of the opinion the $\varphi_{s}$ must increase with approach to the equator, but admits that there are no reliable modern data to verify this hypothesis. Ekman's theory is, in part, based on observations of drift currents in the surface layers of the ocean. Ekman emphasizes that his conclusions are tentative; an inspection shows that his analysis is not everywhere correct dimensionally.

Table 5 demonstrates very clearly that the variation of $\varphi_{s}$ with $f$ is very slight in all latitudes except the immediate vicinity of the equator. At these very low latitudes, on the other hand, it is necessary to take into consideration the large percentual variations in the Coriolis' parameter and the accelerations caused thereby. Thus it becomes practically impossible to verify this phase of the theory.

We have shown that the total range in $\varphi_{s}$ for normal values of $f$ and $U_{\theta}$ is very small. The roughness parameter $z_{0}$, on the other hand, varies over a great range and may therefore cause larger variations in $\varphi_{s}$. Hann ${ }^{19}$ has compiled a number of the early determinations of $\varphi_{s}$; they seem to indicate that $\varphi_{s}$ increases with the continentality of the station. Unfortunately, most of these values are based on the regular morning observations, taken at a time when the atmosphere as a rule is characterized by a high degree of stability. It will be shown later that stability of stratification generally has a tendency to increase $\varphi_{s}$ considerably. Since the stability increases with the continentality of the station it is quite possible that the high values of $\varphi_{s}$ found by some of the earlier investigators (for instance Loomis) for the interior of the continents simply indicate the effect of stability.

Van Everdingen ${ }^{20}$ has published some data which seem to be free from the objections raised above. His gradient wind directions are determined from triangles of barometric stations; all cases of pressure gradients less than $1 \mathrm{~mm}$. Hg per $100 \mathrm{~km}$. are excluded. The individual observations have been accorded weights proportional to the corresponding surface wind velocity. Thus one may assume that the influence of stability has been eliminated from van Everdingen's data, although they are based on the 0700 (GMT) observations. The results are given in Table 8.

$\begin{array}{lcccc} & & \text { TABLE } 8 & \text { Berlin } \\ \text { Station } & \text { De Bilt } & \text { Aachen } & \text { Potsdam } & \text { Bom } \\ \text { Period } & 1905^{-1} 1^{\circ} & 1906-1906 & 1903 & 27^{\circ} \\ \varphi_{s} & 21^{\circ} & 31^{\circ} & 30^{\circ}\end{array}$

Assuming the average height of buildings in a large European city to be around $30 \mathrm{~m}$., one would expect the roughness parameter $z_{0}$ to have a value of about $100 \mathrm{~cm}$. In middle latitudes and for moderate gradient winds $\left(U_{g}=\mathrm{I} 2 \mathrm{~m}\right.$.p.s., $\left.f=\mathrm{I} \cdot 2 \cdot 1 \mathrm{O}^{-4}\right)$ this would give us a value of $N=10^{5}$ and a value of $\varphi_{s}$ in the vicinity of $28^{\circ}$, in fair agreement with the observations quoted in Table 8. It will be shown later that a refinement of the theory leads to a slightly different relation between $N$ and $\varphi_{s}$ (curve marked second approximation in Fig. 7). This second curve indicates a $\varphi_{s}$-value of about $32^{\circ}$ for $N=10^{5}$. The observed values are enclosed by these two limits.*

\footnotetext{
* It is possible that the effective, eddy-producing roughness is materially less than $30 \mathrm{~m}$. Assuming $z_{0}=50 \mathrm{~cm}$, the corresponding $\varphi_{s}$-values are $26^{\circ}$ and $30^{\circ}$, in reasonable agreement with van Everdingen's data. This last value for $z_{0}$ agrees fairly well with the results of the analysis of the Boston aerological material $(\mathrm{II}, 3)$.
} 
We shall now compute the relation between the surface wind velocity and the total height $H+h$ of the layer of frictional influence. At the level of the anemometer $\left(z_{a}\right)$ one has

$$
W_{a}=\frac{\mathrm{I}}{k_{0}} \sqrt{\frac{\overline{\tau_{0}}}{\rho}} \ln \frac{z_{a}+z_{0}}{z_{0}} .
$$

The total height of the layer of frictional influence is obtained from a combination of (22) and (26),

$$
H+h=\mathrm{I} . \mathrm{I} 2 h=\frac{3 \cdot 36 k}{f} \sqrt{\frac{\tau_{0}}{\rho}}
$$

or, from (A 167 ),

$$
H+h=\mathrm{I} . \mathrm{I} 2 h=\frac{5 \cdot 0_{4} k^{2}}{f} U_{g} \sin \varphi_{s} .
$$

Combining (4) and (32a) we find

$$
H+h=\frac{3.36 k k_{0}}{f} \frac{W_{a}}{\ln \frac{z_{a}+z_{0}}{z_{0}}}=246 \frac{W_{a}}{\sin L \log \frac{z_{a}+z_{0}}{z_{\phi_{0}}}} \cdot 5 c_{c}
$$

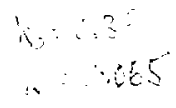

Thus the height of the layer of frictional influence is directly proportional to the wind velocity at anemometer level and inversely proportional to the sine of the latitude. For given values of $W_{a}$ and of $f$ it increases with increasing roughness, $z_{0}$. The relation (33) is plotted below in Fig. II.

In the-next section we shall attempt to verify the theoretical relation (33) between the height of the layer of frictional influence and the surface wind velocity.

The ratio between the wind speed at anemometer level and the gradient wind speed is easily computed. Combining (22) and (A 167) we obtain

$$
\sqrt{\frac{\tau_{0}}{\rho}}=\frac{3 k}{2} U_{\diamond} \sin \varphi_{s}
$$

If we eliminate $\sqrt{\tau_{0} / \rho}$ from (4) with the aid of (34) it follows that

$$
\frac{W_{a}}{U_{o}}=\frac{3 k}{2 k_{0}} \sin \varphi_{s} \cdot \ln \frac{z_{a}+z_{0}}{z_{0}}
$$

Using Taylor's data (Table 7) for strong winds $\left(U_{g}=15.6\right.$ m.p.s., $\left.\varphi_{s}=20^{\circ}\right)$ and the $z_{0^{-}}$ value characteristic of open grass land $\left(z_{0}=3.2 \mathrm{~cm}\right.$.) it follows that

$$
\frac{W_{a}}{U_{\theta}}=0.595 \quad, \quad\left(z_{a}=30 \mathrm{~m} .\right)
$$

in excellent agreement with the observed value of $0.6 \mathrm{I}$.

If we now proceed to a station in a rougher landscape, keeping $U_{g}$ and $f$ constant, the value of $W_{a} / U_{\vartheta}$ will change because of changes in $\varphi_{s}$ and $z_{0}$. Increasing the roughness 
to $3.2 \mathrm{~m}$., corresponding to rough, hilly country, we find for $\varphi_{s}$ a value of $3 \mathrm{I}^{\circ}$. In this case, using the same value as before for $z_{a}(3 \circ \mathrm{m}$.$) ,$

$$
\frac{W_{a}}{U_{B}}=0.293
$$

showing that $W_{a} / U_{g}$ varies in the correct sense with the roughness of the ground.

\section{Light Winds and Residual Turbulence}

It was mentioned above that the ideal frictional layer is found in initially slightly stable air masses which are stirred by moderate or strong winds until they assume an adiabatic temperature distribution up to the equilibrium level $H+h$. The amount of the resulting temperature inversion at the top of the homogeneous layer increases with the initial stability and with the height $H+h$, i.e. with the gradient wind velocity.

The rapid diffusion of heat and moisture in the atmosphere indicates that also those strata that are located above the layer of frictional influence must harbor a certain amount of eddy motion. The energy source of this residual or permanent eddy motion may be found partly in convection and condensation phenomena, partly in shearing forces caused by vertical variations in the horizontal pressure gradient. The residual turbulence varies from place to place and from time to time. Its intensity will depend upon the rate of decay of eddy motion due to molecular viscosity. In the absence of a rational theory for the residual eddy-viscosity at higher levels it seems advisable to assign to it a certain constant mean value $\left(\eta_{r}\right)$. From a study of the many $\eta$-determinations compiled by Richardson it appears reasonable to assume

$$
\eta_{r}=50 \text { c.g.s. units. }
$$

No other claim is made for this numerical value than that it fairly well represents the order of magnitude of the residual eddy-viscosity under normal, stable conditions. In unstable air currents (fresh outbreaks of polar maritime air) the eddy-viscosity aloft may be ten to perhaps a hundred times larger.

With the aid of the above value for $\eta_{r}$ it is easily seen that stresses originating at higher levels due to vertical changes in the gradient wind must normally be small when compared with the ground stress produced by a moderate or fresh wind. Furthermore, the frictional layer is protected by a marked upper inversion of low eddy-viscosity; stresses from above are not readily transmitted through this layer. These circumstances favor the development of an ideal layer of frictional influence.

Light winds have different characteristics. The protecting inversion above their frictional layer is likely to be somewhat less developed and is therefore more capable of transmitting stresses from above. These upper stresses may be of the same order of magnitude as the ground stress.

Fig. 9 represents the theoretical distribution of $\eta$ as a function of $z /(H+h)$. Curves have been drawn for two different values of $U_{s}, 7.5$ and 20 m.p.s. The curves show that the theoretical maximum value of $\eta$ approaches the value of the residual eddy-viscosity as the gradient wind velocity decreases and may even become less for sufficiently weak pressure gradients. 
In the same figure we have indicated, by broken lines, the probable general character of the true $\eta$-curves. When the gradient wind is strong there must be a pronounced minimum in $\eta$ at the level of the frictional inversion. For weak gradients this inversion becomes slightly less distinct and consequently the minimum in $\eta$ is somewhat less pronounced. The curves indicate that light winds are characterized by practically constant $\eta$-values above the boundary layer $H$.

Ekman and Taylor have given the general theory for a frictional layer of constant eddy-viscosity, but their treatment is incomplete in the discussion of surface conditions. Taking into consideration condi-

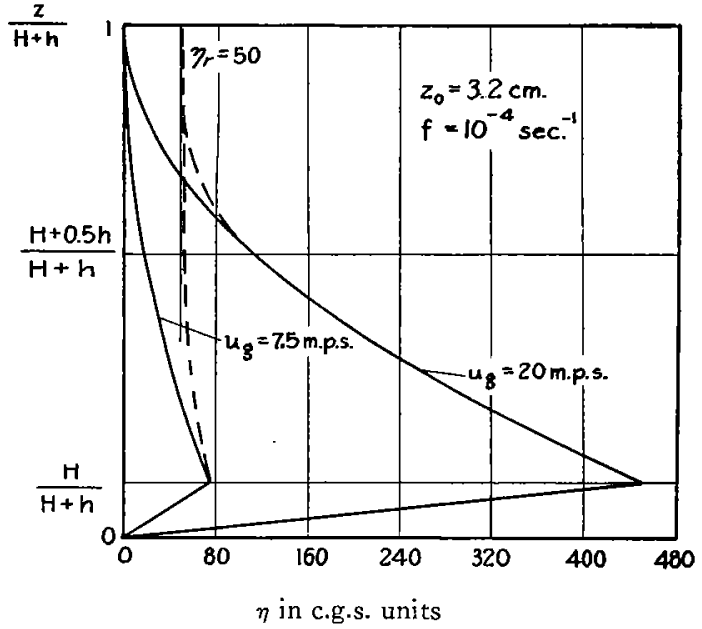

FIG. 9.-Theoretical distribution of eddy-viscosity. tions next to the ground, where the velocity distribution must be of the Prandtl type, it is possible to obtain a definite expression for $\varphi_{s}$.

If we indicate by $a$ the quantity

$$
a=\sqrt{\frac{\rho f}{2 \eta_{r}}}
$$

Ekman's solution may be written in the form

$$
(U+i V)-U_{\vartheta}=\left(W_{H} e^{i \varphi_{s}}-U_{\theta}\right) e^{-a(1+i) z} .
$$

Between the wind at the lower boundary (i.e. height $H$ ) and the gradient wind exists a relation

$$
W_{H}=U_{g}\left(\cos \varphi_{s}-\sin \varphi_{s}\right) .
$$

It follows from (37) that the rate of shear at the lower boundary is given by

$$
C \equiv \sqrt{\left(\frac{d \bar{U}}{d z}\right)^{2}+\left(\frac{d V}{d z}\right)^{2}}=2 a U_{o} \sin \varphi_{s}
$$

Thus the stress at the surface is given by

$$
\frac{\tau_{0}}{\rho}=\sqrt{\frac{2 f \eta_{r}}{\rho}} U_{\theta} \sin \varphi_{s}
$$

In (37), the vertical coordinate $z$ is counted from the upper boundary of the shallow surface layer $H$, within which the velocity distribution is of the Prandtl type. We require continuity in stress, in wind direction and wind velocity and in rate of shear at this level. It follows from these conditions that $\eta$ is continuous. 
The condition of continuity in rate of shear may be written in the form

$$
2 a U_{g} \sin \varphi_{s}=\frac{\mathrm{I}}{k_{0}\left(H+z_{0}\right)} \sqrt{\frac{\overline{\tau_{0}}}{\rho}} .
$$

Making use of the condition of continuity in stress, we may write the above equation in the form

$$
2 a U_{g} \sin \varphi_{s}=\frac{\mathrm{I}}{k_{0}\left(H+z_{0}\right)} \sqrt[4]{\frac{2 \overline{2 \eta_{r}}}{\rho}} \sqrt{U_{\theta} \sin \varphi_{s}}
$$

or, after some reductions,

$$
\frac{H+z_{0}}{z_{0}}=\frac{E^{3 / 4}}{k_{0} \sqrt[3]{2} \sqrt{N} \sqrt{\sin \varphi_{s}}}, \quad\left(N=\frac{U_{g}}{f z_{0}}\right)
$$

where the symbol $E$ represents a pure number defined by

$$
E=\frac{\eta_{r}}{\rho f z_{0}^{2}} .
$$

Assuming $\eta_{r}=50, \rho=\mathrm{I} .25 \cdot \mathrm{IO}^{-3}, z_{0}=3.18 \mathrm{~cm}$. and $f=\mathrm{I} . \mathrm{I} 2 \cdot 1 \mathrm{O}^{-4}$, we obtain

$$
E \sim 4 \cdot{ }^{10} \text {. }
$$

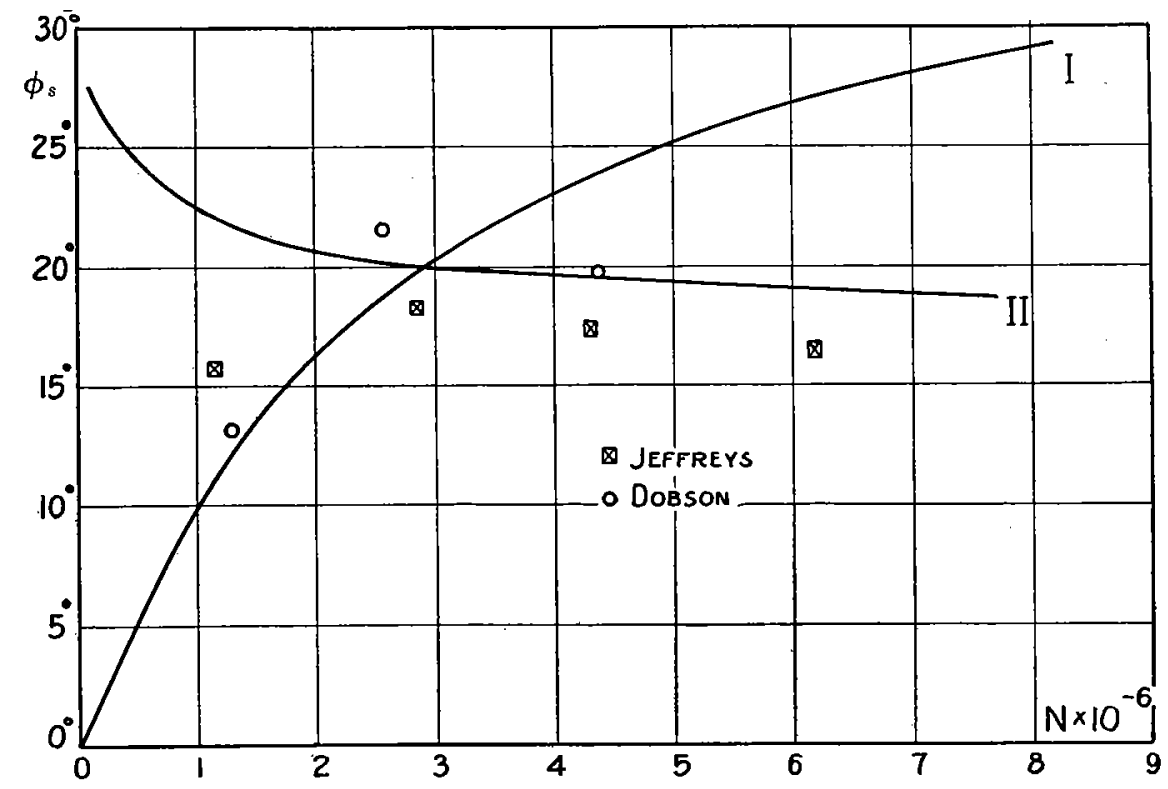

Fig. ro.- Relation between $\varphi_{s}$ and $N$ for two different assumptions regarding the vertical distribution of $\eta$.

From the condition of continuity in wind velocity it follows that

$$
U_{s}\left(\cos \varphi_{s}-\sin \varphi_{s}\right)=\frac{\mathrm{I}}{k_{0}} \sqrt{\frac{\overline{\tau_{0}}}{\rho}} \ln \frac{H+z_{0}}{z_{0}} .
$$


With the aid of expression (40) for $\tau_{0}$ and equation (42) for $H+z_{0}$ this changes into

$$
k_{0} \sqrt[4]{8} \frac{\sqrt{N \sin \varphi_{s}}}{\sqrt[4]{E}}\left(\cot \varphi_{s}-\mathrm{I}\right)=\frac{3}{2} \ln E-\ln k_{0}{ }^{2} \sqrt{2}-\left(\ln N \sin \varphi_{s}\right),
$$

from which equation $\varphi_{s}$ may be determined provided we know the two numbers $N$ and $E$.

Equation (45) is represented graphically in Fig. Io for the above mentioned value $E=4 \cdot 10^{7}$ (curve I). In the same diagram we have entered the relation between $N$ and $\varphi_{s}$ given by our original theory (curve II). Dobson's and Jeffreys' observed values of $\varphi_{s}$ have been entered and plotted against $N$ with the aid of the relation

$$
N \equiv \frac{U_{o}}{f z_{0}}=2.8 \mathrm{I} \cdot \mathrm{IO}^{3} U_{g} \quad\left(f=\mathrm{I} . \mathrm{I} 2 \cdot \mathrm{IO}^{-4}, z_{0}=3 . \mathrm{I} 8 \mathrm{~cm} .\right) .
$$

Both sets of observations definitely indicate the existence of a maximum value of $\varphi_{s}$ for moderate wind velocities, in good agreement with the theoretical prediction.

\section{A Study of the Homogeneous Layer at Boston}

The theory given above indicates a definite upper limit to the layer of frictional influence, designated by the height $H+h$. This is to be identified with the well-known homogeneous or turbulent layer found in many meteorograph records, characterized by nearly adiabatic lapse rate with a sharp decrease in lapse rate or inversion at the top, and by uniform specific humidity; and in many cases, at least, obviously produced by frictional turbulence.

Of course the theory gives the steady-state solution on the assumption of a preexistent adiabatic atmosphere, and hence says nothing about a discontinuity of lapse rate at the height $H+h$. But if at any time the homogeneous layer is shallower than the theoretical $H+h$, the condition is an unsteady one in which finite values of the eddyviscosity coefficient, and hence of Austausch, exist at the top, with the result that mixing is taking place and the layer being extended upward. In a short time, under ordinary conditions, the layer has extended to nearly its steady-state thickness and the velocity (etc.) distribution of the theory obtains throughout. Since the normal tendency (aside from frictional considerations) is toward a lapse rate considerably less than adiabatic, the homogeneous layer is generally confined to the layer of frictional influence, and yet is maintained at approximately the steady-state height of the latter as indicated.

The theory shows that $H+h$ is proportional to the gradient-wind speed and depends further on the parameters $L$ and $\varphi_{s}(32 \mathrm{~b})$, or that $H+h$ is proportional to the wind speed at anemometer level and depends further on $L$ and $z_{0}$ (33). The latter relation is shown graphically in Fig. II. This expression especially provides a convenient check on the theory. The thickness of the homogeneous layer and $W_{a}$ are easily measured. The major purpose of this section is to determine whether they are proportional for a given locality. If this is so, then we can determine whether the proportionality factor gives a reasonable value of $z_{0}$.

There are of course other factors which affect the thickness of the frictional layer in individual meteorograph records. Active convection resulting from marked instability in lower levels may extend the layer greatly beyond its normal height. On the other hand a 
large inversion near the ground prevents its development to normal height. Hence the individual observations must show a large amount of scattering; but since these extraneous factors do not all act in the same direction, it is likely that the means over a large number of observations depend chiefly on the frictional influence. It is on this basis that the following analysis was made.

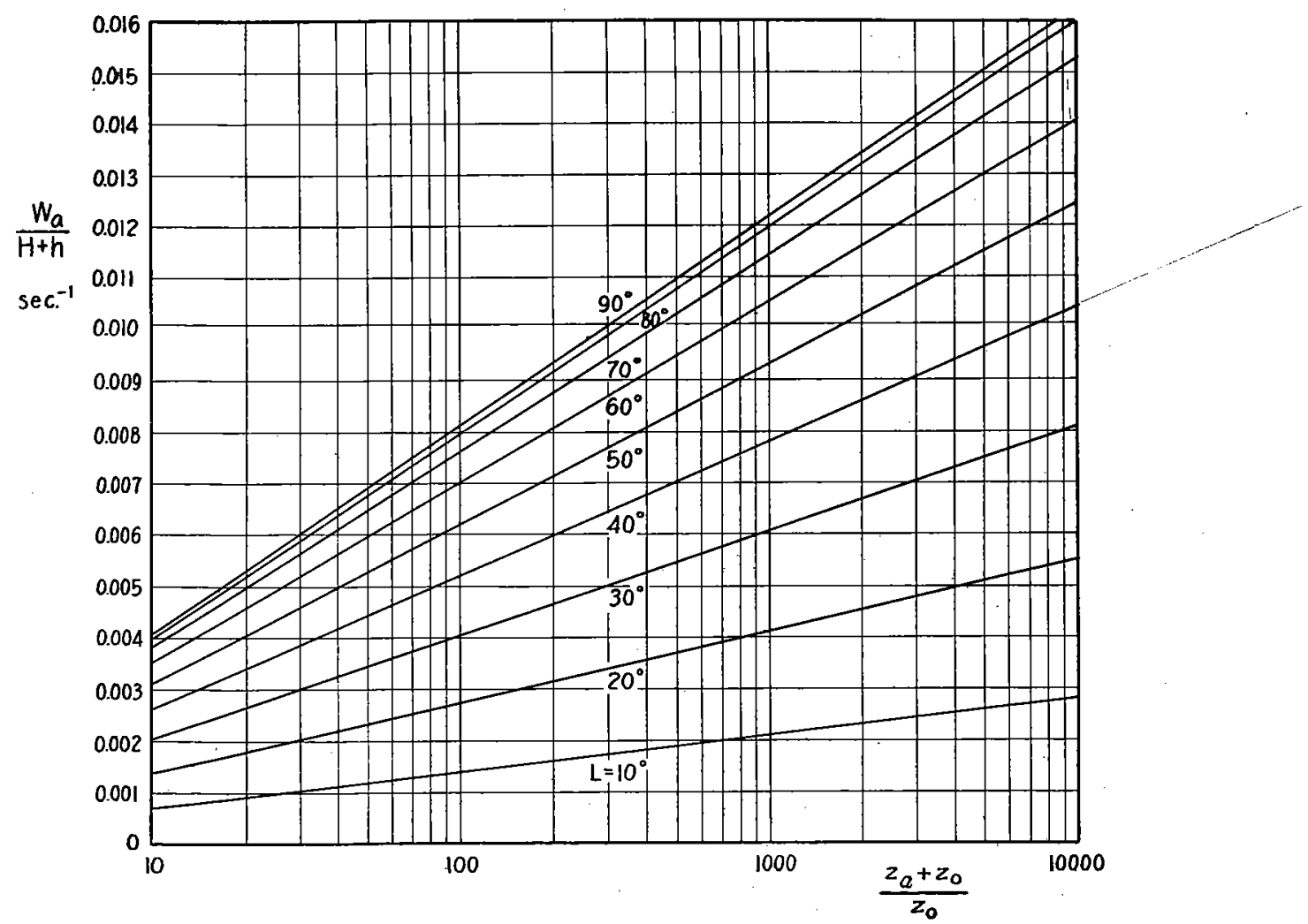

FIG. II.-Theoretical dependence of the height of the homogeneous layer $(H+h)$ on surface wind $\left(W_{a}\right)$, latitude and roughness $\left(z_{0}\right)$.

The study is based on the meteorograph records from the daily airplane ascents made from East Boston Airport (Lat. $42^{\circ} 22^{\prime} \mathrm{N}$, Long. $71^{\circ} 2^{\prime} \mathrm{W}$ ) by M. I. T., for the period November 17, I931, to December 26, I933.

For each flight the following information was tabulated:

Date and time (75th meridian) of take-off.

Wind direction and velocity in m.p.s. as observed from the Weather Bureau's anemometer atop the airport building, I 5 meters above the field.

The altitude in meters of the first minimum temperature on the meteorograph trace, $H+h$. The base of an isothermal layer not starting at the ground was taken as $H+h$. For a ground inversion more than $\mathrm{I} 00 \mathrm{~m}$. deep, $H+h=0$. The homogeneous layer was for convenience defined in this way.

The altitude of the first maximum temperature, or of the top of the isothermal layer, above $H+h, H_{i}$. 
The potential temperature at the ground, at $H+h$, and at $H_{i}$, in degrees centigrade.

From this was computed the potential temperature gradient in the homogeneous layer, $\theta^{\prime}$, and in the inversion, $\theta_{i}{ }^{\prime}$, in degrees centigrade per $100 \mathrm{~m}$. Average values of $H+h, \theta^{\prime}$, $\theta_{i}{ }^{\prime}$ were formed according to each of the following divisions:

Season: October-November, December-January, February-March, April-May. Time of day: early morning (370 flights), later in the day (I35 flights).

Wind direction: NNE-ESE, SE-WSW, W-N.

Wind speed: each Beaufort unit.

The following simplified Beaufort system, which is used at M. I. T., was employed:

$\begin{array}{ccc}\text { Force } & \text { miles/hr. } & \text { m.p.s. } \\ 0 & 0 & 0 \\ \text { I } & \mathrm{I}-4 & \mathrm{I}-2 \\ 2 & 5-9 & 3-4 \\ 3 & \text { IO-I } 4 & 5-6 \\ 4 & \mathrm{I} 5-\mathrm{I} 9 & 7-8 \\ 5 & 20-24 & 9-10 \\ 6 & 25-29 & \mathrm{II}-\mathrm{I} 3 \\ 7 & 30-34 & \mathrm{I} 4-\mathrm{I} 5 \\ 8 & 34-39 & \mathrm{I} 6-\mathrm{I} 7\end{array}$

These data are shown in Tables 9, ro and I I. Each box gives the average of $H+h$, $\theta^{\prime}$, or $\theta_{i}{ }^{\prime}$, for the early morning or the later flights, for a single season, and for a single wind force. Within the box the upper figure is for NNE-ESE, the middle figure for SEWSW, the bottom figure for W-N. Each figure is followed, after the hyphen, by the number of observations on which it is based. The single figure on the right in the box is the weighted mean of those on the left.

The time of the early morning and later flights fall within the following limits:

October-November
December-January
February-March
April-May

October-November

February-March

April-May

Early morning
$0730-0910$
$0738-0859$
$0742-0912$
$0708-0850$

\section{Later}

$0932-1706$

$0900-1640$

$0855^{-1742}$

Og00-I 742

This material is not altogether satisfactory. In the first place $H+h$ as defined for the observations in many cases does not really represent the thickness of the homogeneous layer. Its top is not always marked by an inversion, but may show only a decrease in lapse rate or perhaps only a change in specific humidity. For this reason many of the values are undoubtedly too high. In the second place, there are cases where, though $H+h$ is truly the thickness of the layer, it is not a characteristic thickness. For instance there may have been a rapid diminution of wind, so that $H+h$ really corresponds to a stronger wind than that at the time of the flight. It might have been better to use only flights which clearly showed a pure turbulent layer. But this would have greatly reduced the amount of material available and also have introduced a large personal element, so it seemed preferable to use all the flights and define $H+h$ so that it could be determined in a purely "mechanical" manner, trusting to the large number of observations to counteract individual unsatisfactory ones. 
TABLE 9

$H+h$ in Meters

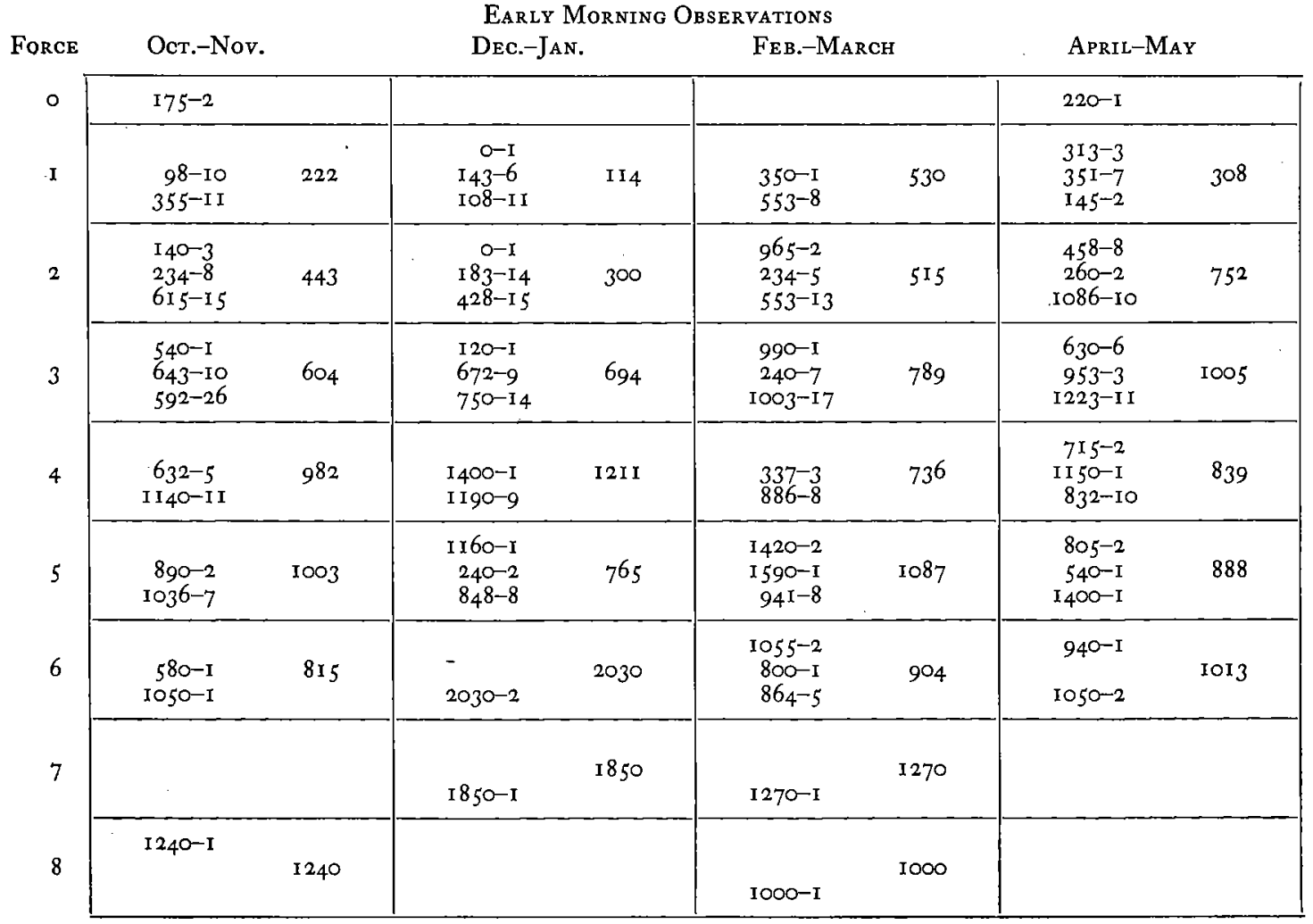

Late Observations

\begin{tabular}{|c|c|c|c|c|c|c|c|c|}
\hline$\circ$ & & & & & & & & \\
\hline I & & & $\begin{array}{l}330-6 \\
100-7\end{array}$ & 206 & $\begin{array}{l}820-2 \\
120-2\end{array}$ & 470 & & \\
\hline 2 & $\begin{array}{l}580-1 \\
780-4 \\
8 \mathrm{I} 3-3\end{array}$ & 768 & $\begin{array}{c}\mathrm{O}-\mathrm{I} \\
280-\mathrm{II} \\
5 \mathrm{I} 3^{-12}\end{array}$ & $3^{8} 5$ & $\begin{array}{r}450-1 \\
1030-2 \\
2350-1\end{array}$ & 1215 & $\begin{array}{l}280-2 \\
460-I\end{array}$ & $34^{\circ}$ \\
\hline 3 & $\begin{array}{r}70-1 \\
615-2 \\
915^{-2}\end{array}$ & 626 & $\begin{array}{l}\text { I } 20-1 \\
963-3 \\
735-6\end{array}$ & 742 & $\begin{array}{l}595-6 \\
903-3\end{array}$ & 698 & $\begin{array}{r}90-1 \\
670-1 \\
950-1\end{array}$ & $57^{\circ}$ \\
\hline 4 & $\begin{array}{l}960-1 \\
950-I\end{array}$ & 955 & $\begin{array}{l}\text { I } 400-I \\
\text { I } 328-5\end{array}$ & I 340 & $\begin{array}{r}325^{-2} \\
940^{-2} \\
1488-5\end{array}$ & 1108 & $\begin{array}{r}356-5 \\
2180-1 \\
1215^{-2}\end{array}$ & 924 \\
\hline 5 & $950-I$ & 950 & $\begin{array}{l}750-1 \\
914-7\end{array}$ & 894 & $\begin{array}{l}1600-I \\
1243-4\end{array}$ & $\mathrm{I}_{3} \mathrm{I}_{4}$ & & \\
\hline 6 & $\begin{array}{r}1430-1 \\
2190-1 \\
500-1\end{array}$ & I 373 & $\begin{array}{l}x 830-1 \\
1707-3\end{array}$ & I738 & $\begin{array}{l}1050-I \\
1400-I\end{array}$ & 1225 & $\begin{array}{r}580-1 \\
1080-1\end{array}$ & 830 \\
\hline 7 & & & & & $2420-I$ & 2420 & & \\
\hline 8 & & & & & & & $500-1$ & 500 \\
\hline
\end{tabular}


TABLE 10

$\theta^{\prime}$ in Degrees Centigrade Per ioo Meters

\begin{tabular}{|c|c|c|c|c|c|c|c|c|}
\hline \multirow{3}{*}{$\begin{array}{c}\text { ForCE } \\
\circ\end{array}$} & \multicolumn{7}{|c|}{ Earluy Morning Observations } & \\
\hline & \multicolumn{2}{|c|}{ Oct.-Nov. } & \multicolumn{2}{|c|}{ Dec.-JAN. } & \multicolumn{2}{|c|}{ FEB.-MARch } & \multicolumn{2}{|c|}{ APRIL-MAY } \\
\hline & $.54^{-1}$ & & & & & & $\cdot 4^{\mathrm{I}-\mathrm{I}}$ & \\
\hline I & $\begin{array}{l}.43-5 \\
.6 \mathrm{I}-9\end{array}$ & .55 & $\begin{array}{l}.50-3 \\
.39-5\end{array}$ & .43 & $\begin{array}{l}.66-1 \\
.32-6\end{array}$ & $\cdot 37$ & $\begin{array}{r}.34-3 \\
-.0 \mathrm{I}-6 \\
-.82-\mathrm{I}\end{array}$ & .02 \\
\hline 2 & $\begin{array}{l}.44^{-2} \\
.53^{-6} \\
.4^{2-13}\end{array}$ & .46 & $\begin{array}{l}\cdot 7 \mathrm{I}-7 \\
.4^{2-1} \mathrm{I} \\
\end{array}$ & .53 & $\begin{array}{l}.00-2 \\
.4 \mathrm{I}-4 \\
.0 \mathrm{I}-13\end{array}$ & .09 & $\begin{array}{c}.08-7 \\
-.15-2 \\
.04-10\end{array}$ & .04 \\
\hline 3 & $\begin{array}{l}.50-1 \\
.27-9 \\
.35-25 \\
\end{array}$ & $\cdot 46$ & $\begin{array}{l}.08-1 \\
.5 x-7 \\
.32-14 \\
\end{array}$ & $\cdot 37$ & $\begin{array}{l}.37-1 \\
.16-6 \\
.18-17\end{array}$ & . I6 & $\begin{array}{c}.05-6 \\
.12-3 \\
-.05-10\end{array}$ & $.0 \mathrm{I}$ \\
\hline 4 & $\begin{array}{l}.55-5 \\
.35-10 \\
\end{array}$ & $\cdot 4^{\mathrm{I}}$ & $\begin{array}{l}.5 \mathrm{I}-\mathrm{I} \\
.18-9 \\
\end{array}$ & .22 & $\begin{array}{l}.3 I-3 \\
.05-8\end{array}$ & .12 & $\begin{array}{r}-.03-2 \\
.04-1 \\
-.01-9\end{array}$ & -.01 \\
\hline 5 & $\begin{array}{l}.39-1 \\
.22-5 \\
\end{array}$ & .25 & $\begin{array}{l}.09-1 \\
.5^{8-I} \\
.26-7\end{array}$ & .28 & $\begin{array}{r}.22-2 \\
-.09-1 \\
.02-8\end{array}$ & .04 & $\begin{array}{r}-.03-2 \\
.19-1\end{array}$ & .05 \\
\hline 6 & $\begin{array}{r}.4^{8-I} \\
-.03^{-I} \\
\end{array}$ & .23 & $. \mathrm{I} 8-\mathrm{r}$ & .18 & $\begin{array}{r}.15-1 \\
.03-1 \\
-.07-5\end{array}$ &.$\infty 0$ & $\begin{array}{c}\cdot 3 \mathrm{I}-\mathrm{I} \\
-.2 \mathrm{I}-2\end{array}$ & -.04 \\
\hline 7 & & & $.04-\mathrm{I}$ & .04 & $.15^{-1}$ &.$x_{5}$ & & \\
\hline 8 & $.27-x$ & .27 & & &.- IO-I & -.10 & & \\
\hline
\end{tabular}

Late Observations

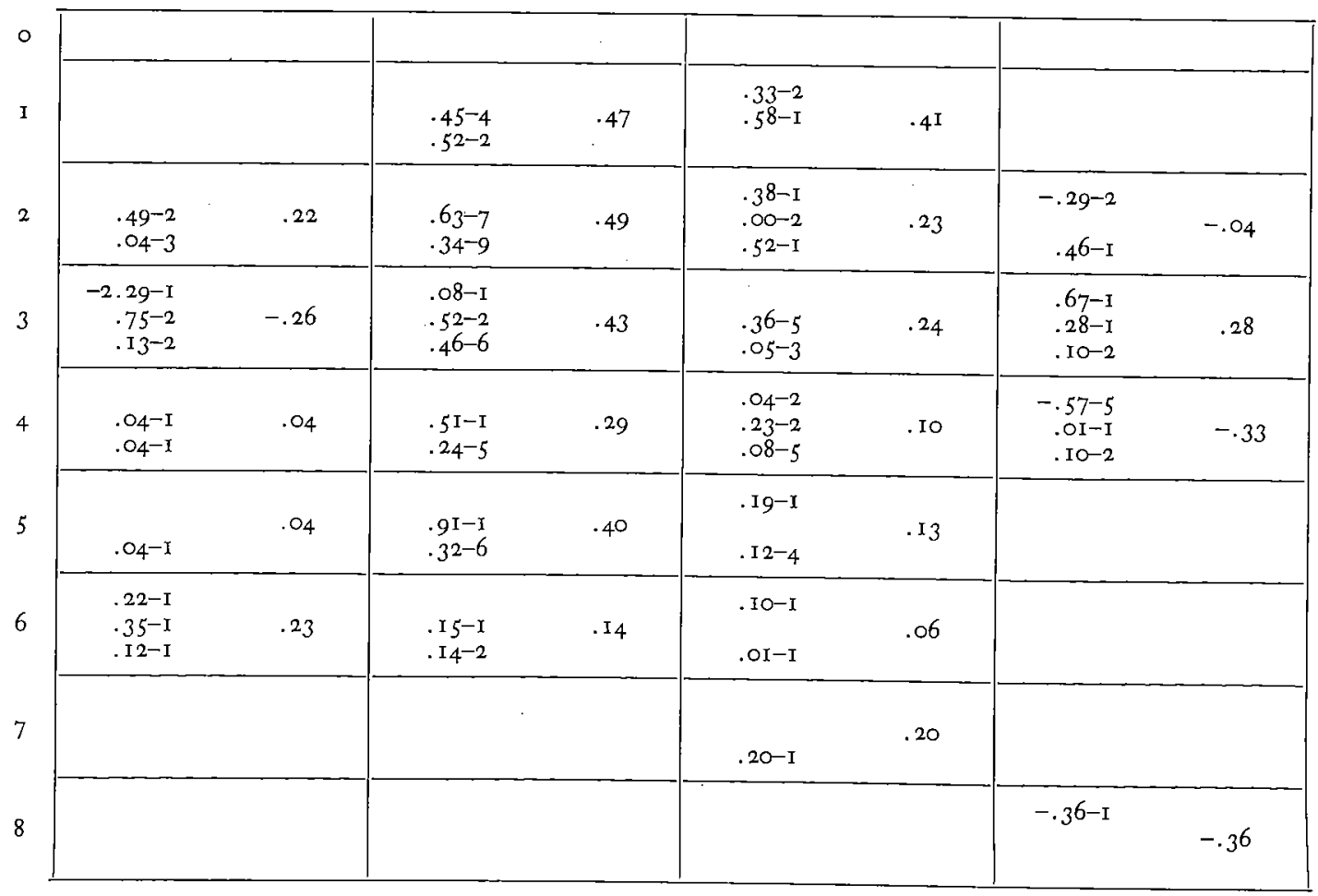


TABLE 11

$\theta_{i}^{\prime}$ in Degrees Centigrade Per ioo Meters

Early Morning Observations

\begin{tabular}{|c|c|c|c|c|c|c|c|c|}
\hline FORCE & \multicolumn{2}{|c|}{ Oct.-Nov. } & \multicolumn{2}{|c|}{ Dec.-Jan. } & \multicolumn{2}{|c|}{$\mathrm{FEB}_{\mathrm{E}}-\mathrm{MARCH}_{\mathrm{A}}$} & \multicolumn{2}{|c|}{ APRIL-M } \\
\hline 0 & I. $93^{-2}$ & & & & & & $2.79^{-x}$ & \\
\hline I & $\begin{array}{l}\text { I. } 77-9 \\
\text { I. } 83^{-9}\end{array}$ & I. 80 & $\begin{array}{l}\text { x. } 93-1 \\
\text { I. } 88-6 \\
2.29-10\end{array}$ & 2.12 & I. $58-6$ & $\mathrm{I} .58$ & $\begin{array}{l}1.60-3 \\
1.47-6\end{array}$ & I. $5 \mathrm{I}$ \\
\hline 2 & $\begin{array}{l}\text { I. } 7 \mathrm{I}-2 \\
x .68-8 \\
2.02-\mathrm{I} 3\end{array}$ & 1.88 & $\begin{array}{l}\text { I. } 45^{-1} \\
\text { I. } 67^{-1} 3 \\
\text { I. } 52-13\end{array}$ & I. 59 & $\begin{array}{l}\text { I. } 53^{-2} \\
\text { I. } 55^{-5} \\
\text { I. } 44^{-1} 3\end{array}$ & I. 48 & $\begin{array}{l}\text { I. } 89-5 \\
2.5^{8-2} \\
1.37-7\end{array}$ & I. 74 \\
\hline 3 & $\begin{array}{l}\text { I. } 70-1 \\
\text { I. } 82-10 \\
2.03^{-21}\end{array}$ & I.95 & $\begin{array}{l}\text { I. } .04-\mathrm{I} \\
\mathrm{I} .8 \mathrm{I}-8 \\
\mathrm{I} .9 \mathrm{I}-\mathrm{I} 3\end{array}$ & I. 83 & $\begin{array}{l}\text { I. } 79-1 \\
\text { I. } 96-6 \\
\text { I. } 66-1.7\end{array}$ & I. 74 & $\begin{array}{l}1.94-6 \\
\text { I. } 50-3 \\
\text { I. } 96-9\end{array}$ & I. 88 \\
\hline 4 & $\begin{array}{l}2.65-5 \\
2.54-10\end{array}$ & 2.58 & $\begin{array}{l}\text { 2. } 10-1 \\
\text { I. } 64-8\end{array}$ & I. 69 & $\begin{array}{l}2.5 x-3 \\
\text { I. } 39-8\end{array}$ & 1.70 & $\begin{array}{l}\text { I. I } 3^{-2} \\
\text { I.05-1 } \\
\text { I. } 7 \text { I-9 }\end{array}$ & I. 56 \\
\hline 5 & $\begin{array}{l}\text { I. 22-2 } \\
2.08-6\end{array}$ & I. 86 & $\begin{array}{l}2.02-1 \\
1.42-2 \\
1.68-7\end{array}$ & I. 66 & $\begin{array}{l}2.76-2 \\
5 \cdot 09-1 \\
\mathrm{I} \cdot 72-8\end{array}$ & 2.21 & $\begin{array}{l}2.00-2 \\
1 \cdot 39-1\end{array}$ & 1.80 \\
\hline 6 & $\begin{array}{l}I .05-I \\
I .4 I-I\end{array}$ & I. 23 & I. I $4^{-I}$ & $\mathrm{I}_{1} \mathrm{I}_{4}$ & $\begin{array}{l}2.5^{8-2} \\
1.33^{-1} \\
2.25^{-5}\end{array}$ & 2.22 & $\begin{array}{l}2.04^{-I} \\
1.9 I-2\end{array}$ & I.95 \\
\hline 7 & & & $2.75^{-1}$ & 2.75 & I . 78-I & I. 78 & & : \\
\hline 8 & $2 \cdot 7^{8-I}$ & $2.7^{8}$ & & & & & & \\
\hline
\end{tabular}

Late Observations

\begin{tabular}{|c|c|c|c|c|c|c|c|c|}
\hline & & & & & & & & \\
\hline & & & $\begin{array}{l}\text { I. } 82-6 \\
2 . \times 2-6\end{array}$ & I. 97 & $\begin{array}{l}2.17^{-2} \\
2.9^{8-2}\end{array}$ & 2.57 & & \\
\hline 2 & $\begin{array}{l}\text { I. } 18-3 \\
\text { I. } 63-3\end{array}$ & $\mathrm{I} \cdot 4^{\mathrm{I}}$ & $\begin{array}{l}\text { I. } 45 \text {-I } \\
\text { I. } 70-10 \\
\text { I. } 46-10\end{array}$ & I. 52 & $\begin{array}{l}\text { I. } 39-1 \\
2.02-2 \\
\text { I. I } 4^{-1}\end{array}$ & I. 55 & $\begin{array}{l}I \cdot 99^{-2} \\
I \cdot 30^{-I}\end{array}$ & 1.76 \\
\hline 3 & $\begin{array}{l}2.36-1 \\
1.82-2 \\
2.12-2\end{array}$ & 2.04 & $\begin{array}{l}\text { I. } 04-I \\
\text { I. } 78-3 \\
\text { I. } 59-6 \\
\end{array}$ & I. 59 & $\begin{array}{l}\text { I. } 48-5 \\
\text { I. } 51-3\end{array}$ & I. 49 & $\begin{array}{l}2.62-I \\
I .04^{-I}\end{array}$ & 1.83 \\
\hline 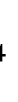 & $I .90-1$ & I.90 & $\begin{array}{l}2.10-1 \\
1.62-4\end{array}$ & I. $7 \mathrm{I}$ & $\begin{array}{l}\text { I. } 76-2 \\
\text { I. } 29-2 \\
\text { I. } 65-5 \\
\end{array}$ & I. 60 & $\begin{array}{l}\text { I. } 71-5 \\
3 \cdot 00-1 \\
\text { I. } 07-2 \\
\end{array}$ & 1.71 \\
\hline 5 & I.90-I & I. 90 & $\begin{array}{l}1 . \infty 0-1 \\
\text { I. } 63^{-6}\end{array}$ & 1.54 & $\begin{array}{l}\text { I. } 75^{-I} \\
\text { I. } 92-4\end{array}$ & 1.89 & & \\
\hline 5 & $\begin{array}{l}2.10-1 \\
3.84^{-I} \\
I .45^{-I}\end{array}$ & $2 \cdot 4^{6}$ & $\begin{array}{l}\text { I. I 5-I } \\
\text { I. I } 4^{-I}\end{array}$ & 1.15 & $\begin{array}{l}3.10-1 \\
1.06-1\end{array}$ & 2.08 & $\begin{array}{l}\text { I. 23-I } \\
\text { I. } 22-I\end{array}$ & I.23 \\
\hline 7 & & & & & I. $87-\mathrm{I}$ & I. 87 & & \\
\hline 8 & & & & & & & I. $24^{-1}$ & $x .24$ \\
\hline
\end{tabular}


The values of $H+h$ in Table 9 show a great deal of scattering, but can be combined in various ways to show the effect, if any, of various parameters. Thus in Fig. 12 are plotted mean values of the early morning flights and of the later flights for each Beaufort unit. Fig. I 3 gives curves for the three sectors of wind direction. Finally Fig. I4 shows mean points for all the observations grouped together. In all these diagrams, the number of observations is shown beside each point.

The last mentioned diagram indicates that the relation between $H+h$ and $W_{a}$ is represented by a straight line, passing

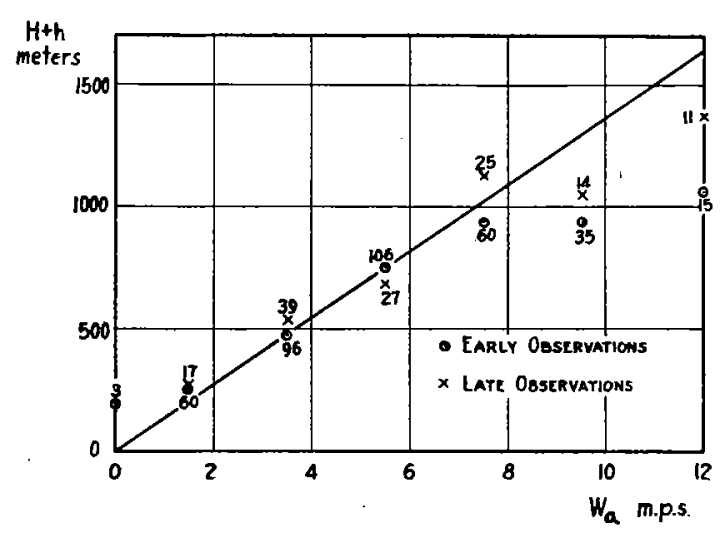

FIG. I 2.-Observations made early in the morning and later in the day, of the height of the homogeneous layer. nearly through the origin, as far as force 4 , after which it falls off somewhat. This is fairly good agreement with the theoretical direct proportionality. The mean of all observations reduced to I m.p.s. (by merely dividing by $W_{a}$ in each case and averaging, omitting only force 0 ) is I36 based on 501 observations. This is represented in Figs. $\mathrm{I}_{2}$ and $\mathrm{I}_{4}$ by the straight line, which has slope $\mathrm{I}_{3} 6$. By

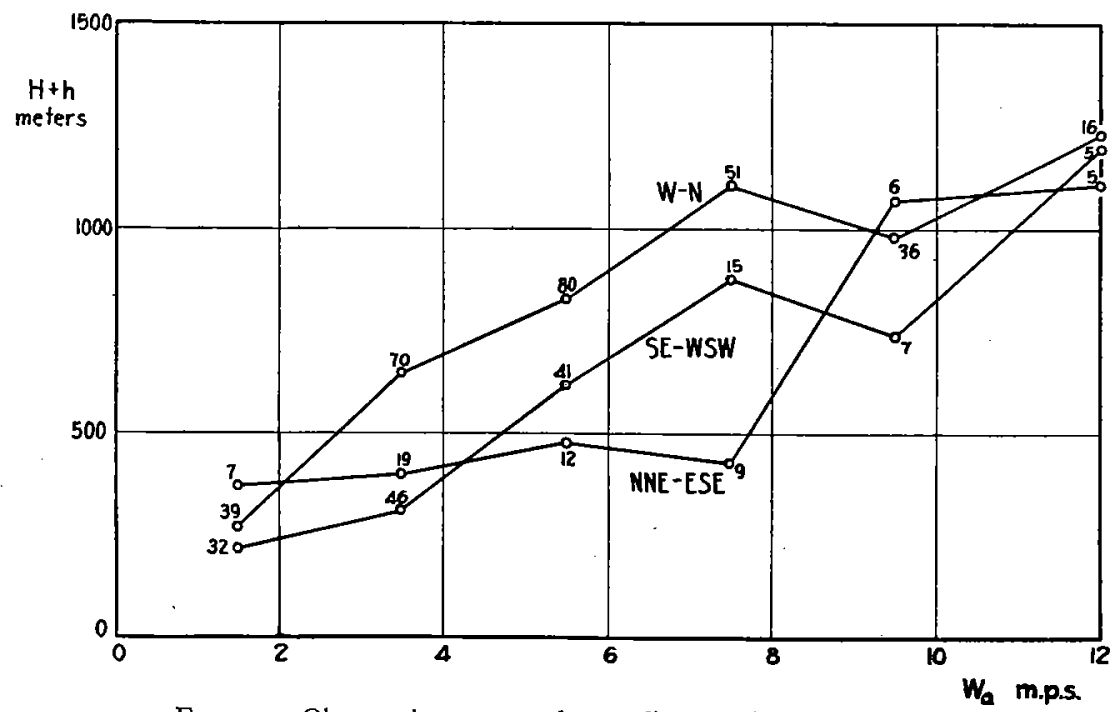

FIG. 13--Observations grouped according to wind direction, of the height of the homogeneous layer.

referring to Fig. I I, since $z_{a}=\mathrm{I} 500 \mathrm{~cm}$., it is seen that $(H+h) / W_{a}=\mathrm{I} 36$ corresponds to a roughness of $z_{0}=3.2 \mathrm{~cm}$., which is a plausible value. Points are also shown in Fig. $\mathrm{I}_{4}$ for the "selected" flights (taken from Table I7), a group including all the flights except those made when the weather map indicated that a front was sufficiently near to prevent the development of the homogeneous layer to its normal height, due either to the confining 
effect of a low frontal inversion or to the variability of wind in the region proximate to the front. The file of analysed weather maps of the M. I. T. Meteorological Course was used for this purpose. It is seen that these "selected" flights give somewhat greater values

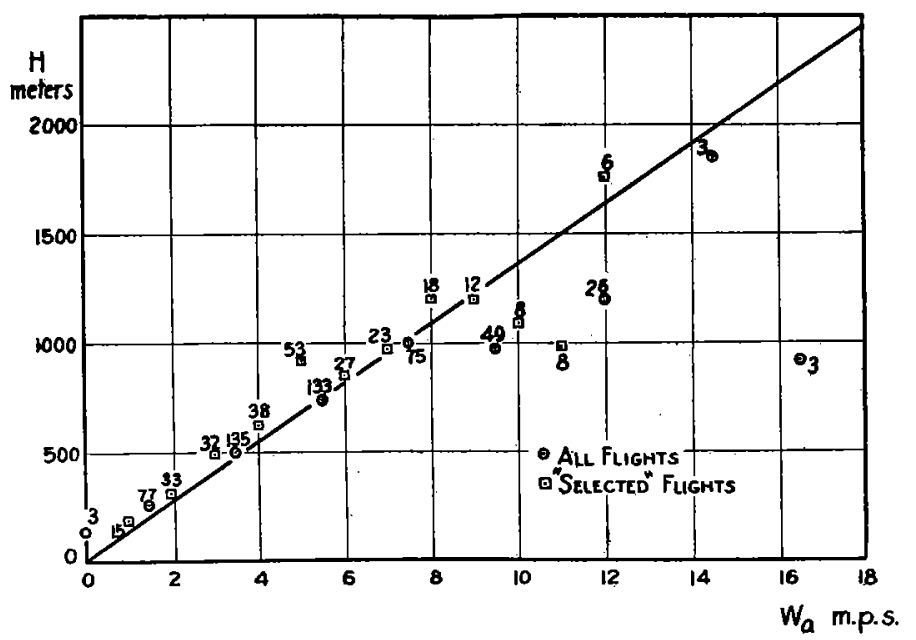

FIG. I 4.-Observations of the height of the homogeneous layer as function of surface wind speed. of $H+h$. The reduction to unit wind speed in this case is 157 based on 273 observations, which corresponds to a roughness of $z_{0}=6 \mathrm{~cm}$. The determinations of $z_{0}$ will be discussed later.

That the observations do not bear out the linear relation between $H+h$ and $W_{a}$ at higher winds than force 4 may be attributed to three effects. In the first place high winds are frequently of too short duration to permit full development of the frictional layer. In the second place, if the layer is not quite at equilibrium, at high winds it is probably too shallow, while at less than average winds it is probably too deep. This also helps to explain the somewhat high values of $H+h$ for forces I and 2 . In the third place, it is natural to expect a relatively steep inversion over a deep homogeneous layer, which greatly hampers any further growth. It is shown in the following table, condensed from the tabulation of $\theta_{i}{ }^{\prime}$ above, that $\theta_{i}{ }^{\prime}$ does increase slightly from force 2 toward higher winds.

TABLE 12

$\theta_{i}{ }^{\prime}$ in Degrees Centigrade per . ioo meters

\begin{tabular}{|c|c|c|c|c|c|c|}
\hline \multirow{2}{*}{$\begin{array}{c}\text { WIND } \\
\text { BEAUFORT }\end{array}$} & \multicolumn{2}{|c|}{ Early Morning } & \multicolumn{2}{|c|}{ LATER } & \multicolumn{2}{|c|}{ ALL } \\
\hline & $\theta_{i}^{\prime}$ & Observations & $\theta_{i}^{\prime}$ & Observations & $\theta_{i}^{\prime}$ & Observations \\
\hline I & I. 83 & 50 & 2.12 & I6 & I.90 & 66 \\
\hline 2 & I. 78 & 84 & I. 53 & 34 & I. $7 \mathrm{x}$ & II 8 \\
\hline 3 & I. 86 & 96 & I. 77 & 25 & I. 84 & I2I \\
\hline 4 & I. 94 & 47 & $x .68$ & 23 & I. 86 & 70 \\
\hline 5 & I. 9 I & 32 & I. $7 \mathrm{I}$ & $x_{3}$ & I. 85 & 45 \\
\hline 6 & I. 94 & $I_{4}$ & $\mathrm{I} .8 \mathrm{I}$ & 9 & 1. 89 & 23 \\
\hline 7 & 2.27 & 2 & 1.87 & I & 2. I3 & 3 \\
\hline 8 & 2.78 & I & $\mathrm{I} .24$ & I & $2.0 I$ & 2 \\
\hline
\end{tabular}

In view of these considerations, we believe that this observational material clearly indicates that the depth of the layer of frictional influence is directly proportional to the wind speed, when other factors do not interfere. It remains to study the effect of some of 
these other factors, and to see whether the proportionality factor gives values of the roughness parameter consistent with the relief. For this latter purpose we have to assume that $s=\mathrm{r} / 3 \mathrm{O}$, which proved successful in the first part of this chapter.

The effect of time of day on the homogeneous layer is shown in Fig. I2. $H+h$ is markedly smaller in the early morning than during the day, which is due to greater stability in the early morning, in particular to occasional ground inversions. This greater stability is clearly shown in the values of $\theta_{i}{ }^{\prime}$ just above.

TABLE 13

$H+h$ IN METERS

\begin{tabular}{|c|c|c|c|c|c|c|c|c|}
\hline \multirow{2}{*}{$\begin{array}{c}\text { WIND } \\
\text { BEAUFORT }\end{array}$} & \multicolumn{2}{|c|}{ Oct.-Nov. } & \multicolumn{2}{|c|}{ Dec.-JAN. } & \multicolumn{2}{|c|}{$\mathrm{F}_{\mathrm{EB}}-\mathrm{M}_{\mathrm{ARCH}}$} & \multicolumn{2}{|c|}{ APRIL-MAY } \\
\hline & $H+h$ & Obs. & $H+h$ & Obs. & $H+h$ & Obs. & $H+h$ & Obs. \\
\hline 0 & I75 & 2 & & o & & o & 220 & I \\
\hline I & 222 & $2 \mathrm{I}$ & I 53 & $3^{\mathrm{I}}$ & 512 & I3 & $3 \circ 8$ & 12 \\
\hline 2 & 519 & 34 & 338 & 54 & 631 & 24 & 698 & 23 \\
\hline 3 & 607 & $4^{2}$ & 708 & 34 & 765 & 34 & 948 & 23 \\
\hline 4 & $97^{8}$ & I8 & I 260 & I6 & 904 & 20 & $87 \mathrm{r}$ & $2 I$ \\
\hline 5 & 998 & IO & 819 & I9 & II 58 & I6 & 868 & 6 \\
\hline 6 & I I 50 & 5 & I 835 & 6 & 968 & IO & $\mathrm{IOI}_{3}$ & 3 \\
\hline 7 & & $\circ$ & 1850 & I & I 845 & 2 & & 0 \\
\hline 8 & I 240 & I & & $\circ$ & 1000 & I & 500 & I \\
\hline$\frac{H+h}{W_{a}}$ & I 28 & I $3 \mathrm{I}$ & 99 & $16 I$ & I 58 & 120 & I6I & 89 \\
\hline
\end{tabular}

The seasonal effect is greater than any other except the wind speed, as shown by Table I3, condensed from Table 9. Not only is the "homogeneous" layer shallower in autumn and early winter, but also less homogeneous, as shown by the tabulated values of $\theta^{\prime}$ for early morning (Table 10). These are much higher during October-January than during February-May. As the day advances, the high values of $\theta^{\prime}$ are wiped out during October and November, but remain during December and January.

It should be noted that all values in Table Io are too high due to lag of the bimetal thermometer. ${ }^{21}$ If this correction is applied to the early morning flights, the lapse rates come closer to adiabatic in October-January, the average for February-March becomes practically purely adiabatic, and the average for April-May becomes slightly superadiabatic. This indicates that our method of determining $H+h$ is quite good for the months February-May and for higher wind speeds in the other months, but that it gives values of $H+h$ larger than actually occur at low winds in October-January. This means that the seasonal effect is even greater than shown by Table I3. Similarly all values in Table I I are somewhat too small.

It is well to comment on the effect of the location of Boston on the values of $H+h$. In many inland localities a strong ground inversion is the rule for winter mornings. For instance the mean winter lapse rate at Ellendale, North Dakota, has an inversion for the 
first 1050 meters. ${ }^{22}$ Likewise $P_{C}$ air masses in winter have an inversion to a mean height (above sea level) of 2 kilometers at Ellendale, Royal Center (Indiana), Broken Arrow (Oklahoma), and Groesbeck (Texas), whereas Boston usually shows no inversion. ${ }^{23}$ Under these abnormally stable conditions it is impossible for the normal frictional layer to develop. That these conditions of marked stability often do occur at Boston is reflected in the lower values of $H+h$ in fall and winter, but they do not prevail as at some other stations, which makes Boston a fortunate choice for this study. The absence of so much stability at Boston can be attributed to the great mixing which occurs as air from the west and north passes over the Berkshires and Green and White Mountains (Ellendale, for instance, lies in quite smooth country), and to the proximity of water which prevents strong radiation cooling at night.

We will discuss the effect of wind direction in detail, since this involves variation in the roughness parameter $z_{0}$. The immediate relief is as follows: The airport itself and the sector from NE to SE is level ground or open water obstructed by a few islands. In the sector from SE to SW water extends about a mile, beyond is moderately level land. But to the southwest is the city of Boston and a few miles in the northwest are hills rising $200-300$ feet. The mountainous regions far to the northwest are probably too distant to have a direct effect on the thickness of the homogeneous layer at Boston; they merely remove very stable conditions which would prevent its normal development.

Fig. I 3 shows that winds W-N give a deeper homogeneous layer than winds SE-WSW or NNE-ESE, the two latter having about equal values on the average. In general, then, there is aggreement with the local relief. In order to obtain values of $H+h$ as typical as possible of the three wind sectors, we have formed the mean of three reductions to unit wind speed. The first is the ordinary arithmetic reduction* of the values in Fig. I3. The second is the least squares reduction of these same values for forces $2,3,4$, formed by computing the quantity $\frac{\Sigma(H+h) W_{a}}{\Sigma W_{a}^{2}}$. The third is the ordinary reduction of the "selected"* flights for each m.p.s. of wind up to I 2 m.p.s. Table $I_{4}$ shows the values for the "selected" flights, and Table I 5 the reduced values and their means.

We will not try to explain the discrepancies between the different types of reductions; we merely assume that the average of the three is more representative than any one would be. The question now to be settled is whether these average reduced values of $H+h$ for different wind sectors can be explained on the basis of the relief in the respective sectors. The corresponding values of $z_{0}$ are found by reference to Fig. Io to be

$\begin{array}{lll} & \frac{H+h}{W_{a}} & z_{0} \\ \text { NNE-ESE } & \text { I Io sec. } & 0.65 \mathrm{~cm} . \\ \text { SE-WSW } & \text { I } 6 & \text { I. } \\ \text { W-N } & \text { I } 58 & 7 .\end{array}$

It must be borne in mind that this method for finding $z_{0}$ is necessarily rough, since $z_{0}$ varies rapidly with $(H+h) / W_{a}$. However, $.65 \mathrm{~cm}$. seems a possible value of the roughness parameter for an airport and protected waters, probably a little too small.

* Cf. p. 3 I. 
TABLE 14

$H+h$ in Meters

\begin{tabular}{|c|c|c|c|c|c|c|}
\hline \multirow{2}{*}{$\begin{array}{c}W_{a} \\
\text { m.p.s. }\end{array}$} & \multicolumn{2}{|c|}{ NNE-ESE } & \multicolumn{2}{|c|}{ SE-WSW } & \multicolumn{2}{|c|}{ W-N } \\
\hline & $H+h$ & Obs. & $H+h$ & Obs. & $H+h$ & Obs. \\
\hline I & 0 & I & 77 & 6 & 309 & 8 \\
\hline 2 & 345 & 2 & 328 & 16 & 297 & I5 \\
\hline 3 & 434 & 7 & 301 & 8 & 601 & 17 \\
\hline 4 & 623 & 6 & $49 \mathrm{I}$ & 9 & 670 & 23 \\
\hline 5 & 975 & 2 & 633 & I5 & 1034 & 36 \\
\hline 6 & 718 & 3 & 885 & 8 & $85 \mathrm{I}$ & 16 \\
\hline 7 & 567 & 3 & 717 & 6 & 1176 & $I_{4}$ \\
\hline 8 & 260 & I & 1283 & 3 & I 248 & 14 \\
\hline 9 & 1830 & 2 & 540 & I & II 34 & 9 \\
\hline 10 & & o & 1283 & 3 & 980 & 5 \\
\hline II & 1230 & I & 1085 & 2 & 894 & 5 \\
\hline I 2 & & $\circ$ & 1607 & 3 & 1913 & 3 \\
\hline
\end{tabular}

TABLE 15

$\frac{H+h}{W_{a}}$ IN SECONDS

\begin{tabular}{|c|c|c|c|c|c|c|c|}
\hline \multirow{4}{*}{$\begin{array}{l}\text { WINd } \\
\text { SECTOR }\end{array}$} & \multicolumn{4}{|c|}{ Arithmetic Reduction } & \multirow{2}{*}{\multicolumn{2}{|c|}{$\frac{\text { LeAst SQUARES Red. }}{3^{-8} \text { m.p.s. }}$}} & \multirow{2}{*}{ Average } \\
\hline & \multicolumn{2}{|c|}{ All } & \multicolumn{2}{|c|}{ "Selected" } & & & \\
\hline & $H+h$ & Obs & $H+h$ & Ohe & $H+h$ & Ohe & $H+h$ \\
\hline & $W_{a}$ & & $W_{a}$ & & $W_{a}$ & Uos. & $W_{a}$ \\
\hline NNE-ESE & $x_{4}$ & 58 & I38 & 28 & 78 & 40 & IIO \\
\hline SE-WSW & I IO & $I_{4} 6$ & I 28 & 80 & rog & 102 & II 6 \\
\hline W-N & $\mathrm{x} 53$ & 292 & I 74 & 165 & I 47 & 201 & 158 \\
\hline
\end{tabular}

The values for the other two sectors have less meaning, since the air has passed over successively smoother surfaces in the last few miles before reaching the airport. They may be said to indicate that the average roughness in the south is fifty per cent greater than in the east, and the average roughness in the northwest ten times as great. Both of these seem too small, and it is especially remarkable that the country to the south is so very much less effective than the hills in the northwest. With southerly winds, the air is modified in passing over a strip of water just before reaching the airport. The redueed friction allows a slightly stronger wind at anemometer level (than if the anemomcter were situated a few miles further south at the same height), and since the distance is too short for $H+h$ to be changed any, $(H+h) / W_{a}$ is decreased appreciably, so that it is 
barely greater than for easterly winds. Air from the northwest, on the other hand, does not pass over a body of water, so that $(H+h) / W_{a}$ is not so greatly reduced as with southerly winds.

We can get an expression for the maximum effect of the immediate smooth surroundings of the airport as follows. We can write

$$
\frac{H+h}{W_{a}}=\frac{H+h}{U_{g}} \frac{U_{g}}{W_{a}}
$$

where the first factor on the right is certainly determined by the roughness in the sector under consideration from $(32 \mathrm{~b})$, and certainly $U_{g}$ is the same in both. Assuming that equilibrium of wind gradient in the lower $15 \mathrm{~m}$. is reached with respect to the immediate surroundings, the second factor is determined approximately by $(35)$, using $z_{0}=.65 \mathrm{~cm}$. Of course (35) does not hold exactly here, because $\varphi_{s}$ must depend in part on the conditions (which have not reached equilibrium) above $15 \mathrm{~m}$., but the approximation is probably good.

Using as a mean value $U_{\theta}=10$ m.p.s., Fig. 8 gives $\varphi_{s}=\mathrm{I} 7.9^{\circ}$, hence $U_{\theta} / W_{a}=1.64$. So from $(32 b)$ we have

$$
\frac{H+h}{W_{a}}=\mathrm{I} .6_{4} \frac{H+h}{U_{a}}=\mathrm{I} .64 \cdot 5.04 \frac{k^{2}}{f} \sin \varphi_{s} .
$$

This is plotted in Fig. I 5 as a function of $z_{0}$ by use of Fig. 8 again. Fig. I 5 gives the following values of the roughness.

$$
\frac{H+h}{W_{a}}
$$

\section{NNE-ESE \\ SE-WSW \\ W-N}

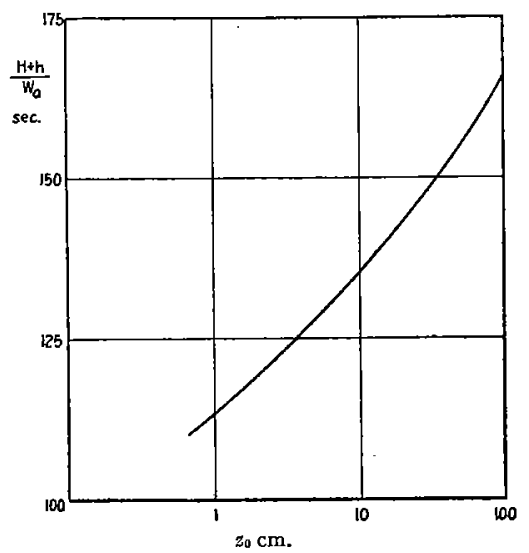

FIG. I 5. - Theoretical height of the homogeneous layer as a function of the roughness of the distant surroundings of the E. Boston Airport.

$\begin{array}{lc}\text { I IO sec. } & 0.65 \mathrm{~cm} . \\ \text { I I } 6 & \text { I. } 5 \\ \text { I } 58 & 60 .\end{array}$

These values are all too low, especially the second; but since, as mentioned above, $z_{0}$ varies rapidly with $(H+h) / W_{0}$, they seem satisfactory enough. It seems that the springtime observations give much better results. Table 13 shows that $(H+h) / W_{a}$ for the months February-May is $159,17 \%$ greater than the annual mean of 136 . If the mean reduced values in Table 15 are increased by $17 \%,(H+h) / W_{a}=\mathrm{I} 29$ for easterly winds, which gives $z_{0}=1.9 \mathrm{~cm}$., nearly three times the value just above, and the $z_{0}$-values for the other sectors are increased almost in the same proportion. These increased values agree well with the relief, which further indicates that during fall and early winter there is sufficient stability to prevent the development of the homogeneous layer to its normal height, rather than that instability extends it beyond its normal height in spring.

$z_{0}$ for southerly winds seems much too small relative 
to the other two; the topography indicates that it should lie more nearly midway between them. This residual effect must be due to greater stability at low levels with southerly winds, which may be the natural result of surface cooling as the air moves toward higher latitudes, or it may be due to the absence of mountainous country such as exists far to the northwest.

We are including here an illustration of a well-developed homogeneous layer as indicated by the vertical temperature distribution. Fig. I6 represents the result of four

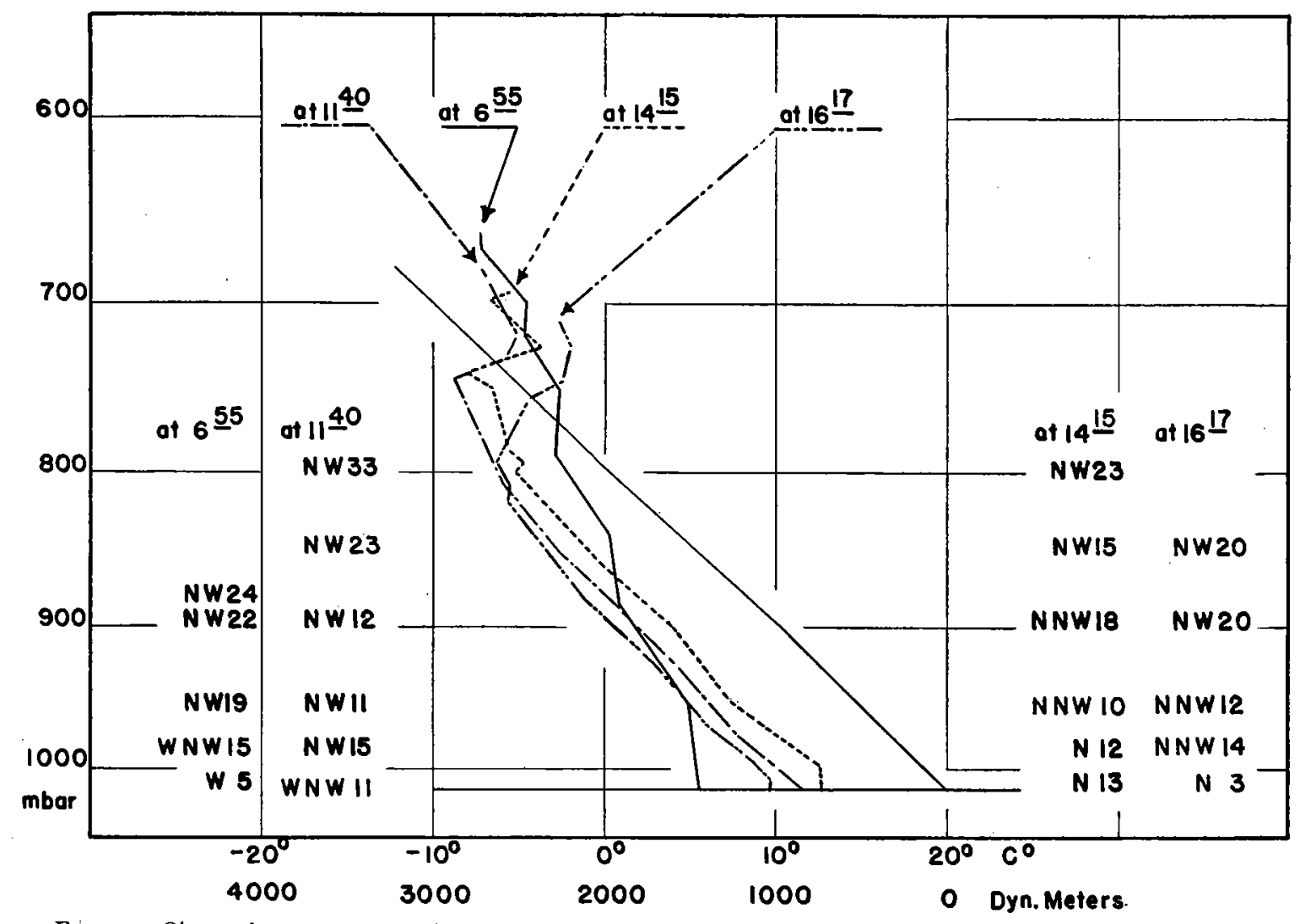

FIG. 15.-Observed temperature and wind distribution over Boston on March 22, I935. The authors are indebted to Dr. K. O. Lange and the pilots of the M.I.T. aerological station for their cooperation in securing these data.

airplane observations in Boston on March 22, 1935. The data are plotted on a Stüve diagram with temperature and the 0.288 th power of pressure as coordinates. The thin diagonal line may be used to determine the heights of the various points in the temperature curves. The early morning flight was made shortly after a cold front passage and reflects the descending motion characteristic of the advancing edge of a cold current. The three remaining flights show adiabatic lapse rates up to a level of about $2 \mathrm{~km}$. It is possible to compute the height of the homogeneous layer with the aid of $(32 \mathrm{~b})$ and the observed wind distribution. We assume the gradient wind to be $23 \mathrm{~m}$.p.s. and the roughness parameter for $\mathrm{NW}$ winds to be $60 \mathrm{~cm}$. This makes $N=3.8 \cdot 10^{5}$ and $\varphi_{s}=24 \cdot 5^{\circ}$. It follows from (32b) that $H+h=2020 \mathrm{~m}$. The observed temperature curves, except the early morning one, show a marked decrease in lapse rate between 1900 and $2000 \mathrm{~m}$. 
From these same meteorograph records we have also determined average values of $H+h$ for each m.p.s. of wind speed from $\mathrm{I}$ to $\mathrm{I} 2$ according to the air mass type present at Boston.

The meaning of the air mass symbols $P_{C}, N_{P C}, N_{P P}, P_{A}, T_{M}$ here used, as well as the characteristics of the air masses themselves are given by Willett. ${ }^{23}$ Under $T_{M}$ are included $T_{G}, T_{A}, N_{T G}, N_{T A}, N_{T M}$, since the air masses represented by these various subdivisions have practically the same properties, and since there are too few examples to study each separately. One case of $\mathrm{N}_{\mathrm{TP}}$ and two of $\mathrm{P}_{\mathrm{P}}$ have been included with $\mathrm{N}_{\mathrm{PP}}$. Two cases of $T_{C}$ have been omitted altogether.

These mean values for all the flights and for the "selected" ones are given in Tables I6 and I7. The values reduced to I m.p.s., according to the three methods, ${ }^{*}$ and their means are shown in Table 18 . This table also gives the average wind direction for each air mass.

TABLE 16

$H+h$ IN METERS

(All Flights)

\begin{tabular}{|c|c|c|c|c|c|c|c|c|c|c|}
\hline \multirow{2}{*}{$\begin{array}{c}W_{a} \\
\text { m.p.s. }\end{array}$} & \multicolumn{2}{|c|}{$\mathbf{P}_{\mathrm{C}}$} & \multicolumn{2}{|c|}{$\mathrm{N}_{\mathrm{PC}}$} & \multicolumn{2}{|c|}{$\mathrm{N}_{\mathrm{PP}}$} & \multicolumn{2}{|c|}{$\mathrm{P}_{\mathrm{A}}$} & \multicolumn{2}{|c|}{$\mathrm{T}_{\mathrm{M}}$} \\
\hline & $H+h$ & Obs. & $H+h$ & Obs. & $H+h$ & Obs. & $H+h$ & Obs. & $H+h$ & Obs. \\
\hline I & 155 & 2 & 262 & I0 & $\circ$ & 4 & & $\circ$ & & $\circ$ \\
\hline 2 & 543 & IO & 239 & I7 & 306 & 18 & 325 & 4 & 40 & 3 \\
\hline 3 & $45^{6}$ & I3 & $5 \pm 4$ & I5 & 323 & IO & 4I 3 & 3 & 540 & 5 \\
\hline 4 & $6 \times 6$ & 23 & 602 & 28 & $63 \mathrm{I}$ & 8 & $34^{\circ}$ & 2 & 360 & 5 \\
\hline 5 & 854 & 30 & $82 I$ & 24 & 824 & I 8 & 520 & 2 & 392 & 5 \\
\hline 6 & 619 & I 8 & 648 & I 3 & 769 & 10 & 560 & I & 948 & 5 \\
\hline 7 & $\ln 16$ & I 2 & 779 & I9 & $87 \mathrm{I}$ & 8 & 697 & 3 & 50 & I \\
\hline 8 & I 386 & I 5 & I 192 & 7 & 820 & 3 & $4 \mathrm{I} 3$ & 3 & & 0 \\
\hline 9 & $94 \mathrm{I}$ & 9 & 858 & 6 & 2100 & $\mathrm{I}$ & 1223 & 3 & 800 & 2 \\
\hline 10 & I 233 & I 3 & 728 & 4 & 410 & 3 & I I 60 & I & & o \\
\hline I I & II 57 & 6 & $133^{\circ}$ & 2 & I 590 & $\mathrm{I}$ & 1060 & $\mathrm{I}$ & 580 & $I$ \\
\hline 12 & 1093 & 6 & 840 & 2 & 2205 & 2 & 730 & 2 & 2190 & 1 \\
\hline
\end{tabular}

By comparison with Table I5, we see that the various air mass types have values of $(H+h) / W_{a}$ corresponding almost exactly with the values for their respective wind directions. Thus for $P_{C}$ the value of $I_{55}$ is the same as for northwesterly winds, those for $N_{P C}$ and $N_{P P}$ are intermediate between the values for northwesterly and southerly winds, and $\mathrm{P}_{\mathrm{A}}$ has a value the same as easterly winds. The greatest discrepancy is between $\mathrm{I} 22$ for $\mathrm{T}_{M}$ and I 16 for winds SE-WSW. This result indicates that the air mass type has a very small effect, if any, on the thickness of the homogeneous layer.

*Cf p. 34 . 
TABLE 17

$H+h$ in Meters

("Selected" Flights)

\begin{tabular}{|c|c|c|c|c|c|c|c|c|c|c|c|c|}
\hline \multirow{2}{*}{$\begin{array}{c}W_{a} \\
\text { m.p.s. }\end{array}$} & \multicolumn{2}{|c|}{$P_{c}$} & \multicolumn{2}{|c|}{$\mathrm{N}_{\mathrm{PC}}$} & \multicolumn{2}{|c|}{$N_{P P}$} & \multicolumn{2}{|c|}{$\mathbf{P}_{\mathrm{A}}$} & \multicolumn{2}{|c|}{$\mathrm{T}_{\mathrm{M}}$} & \multicolumn{2}{|c|}{ Mean } \\
\hline & $H+h$ & Obs. & $H+h$ & Obs. & $H+h$ & Obs. & $H+h$ & Obs. & $H+h$ & Obs. & $H+h$ & Obs. \\
\hline I & I 55 & 2 & $29 x$ & 9 & $\circ$ & 4 & & 0 & & 0 & I95 & 15 \\
\hline 2 & 402 & 5 & 216 & $I_{4}$ & 409 & ro & 420 & 3 & $\circ$ & I & $3 \mathrm{I}_{4}$ & 33 \\
\hline 3 & 565 & IO & 586 & 12 & 195 & 6 & 4I 3 & 3 & 580 & I & 490 & 32 \\
\hline 4 & 659 & I3 & $64 \mathrm{I}$ & 18 & 500 & 3 & 180 & I & 600 & 3 & 621 & $3^{8}$ \\
\hline 5 & 989 & 20 & 964 & 17 & 894 & $x \mathrm{I}$ & 700 & I & 490 & 4 & 9I9 & 53 \\
\hline 6 & $66 x$ & 10 & $74^{2}$ & 6 & 989 & 7 & 560 & I & $\mathrm{I}_{4} 67$ & 3 & 850 & 27 \\
\hline 7 & $\mathrm{I} 246$ & 9 & 677 & 9 & II 90 & 3 & 795 & 2 & & $\circ$ & 977 & 23 \\
\hline 8 & 1379 & II & 1083 & 4 & gro & 2 & 260 & I & & 0 & I I 99 & I 8 \\
\hline 9 & $\mathrm{IOI}_{4}$ & 8 & 540 & $I$ & 2100 & I & 2060 & I & r60o & I & $\mathrm{I} 2 \mathrm{OI}$ & 12 \\
\hline 10 & II 82 & 7 & & $\circ$ & 480 & I & & 0 & & 0 & I094 & 8 \\
\hline I I & 853 & 4 & $\mathrm{I} 23^{\circ}$ & I & I 590 & I & 1060 & I & 580 & I & 984 & 8 \\
\hline I 2 & $\mathrm{I} 320$ & 3 & & 0 & 2205 & 2 & & o & 2190 & I & 1760 & 6 \\
\hline
\end{tabular}

TABLE 18

$H+h$

$\frac{W h}{W_{a}}$ in SECONDS

\begin{tabular}{|c|c|c|c|c|c|c|c|c|}
\hline \multirow{3}{*}{$\begin{array}{l}\text { Air Mass } \\
\text { Type }\end{array}$} & \multicolumn{4}{|c|}{ Arithmettc Reduction } & \multirow{2}{*}{\multicolumn{2}{|c|}{$\begin{array}{c}\text { LEAST SQ. RED. } \\
3^{-8} \text { m.p.s. }\end{array}$}} & \multirow{3}{*}{$\begin{array}{l}\text { AvERAGE } \\
\frac{H+h}{W_{a}}\end{array}$} & \multirow{3}{*}{$\begin{array}{c}\text { MEan Wind } \\
\text { Direction }\end{array}$} \\
\hline & \multicolumn{2}{|c|}{ All } & \multicolumn{2}{|c|}{ "Selected" } & & & & \\
\hline & $\frac{H+h}{W_{a}}$ & Obs. & $\frac{H+h}{W_{a}}$ & Obs. & $\frac{H+h}{W_{a}}$ & Obs. & & \\
\hline$P_{c}$ & I $5 \mathrm{r}$ & 157 & 159 & 102 & I 54 & III & 155 & $N W \times W$ \\
\hline $\mathrm{N}_{\mathrm{PC}}$ & I 44 & $x_{47}$ & 164 & 9 I & I 35 & I06 & $\mathrm{I}_{4} 8$ & $W$ \\
\hline $\mathrm{N}_{\mathrm{PP}}$ & I 34 & 86 & 146 & $5 \mathrm{I}$ & 136 & 57 & 139 & $\mathrm{~W} \times \mathrm{S}$ \\
\hline $\mathrm{P}_{\mathrm{A}}$ & 109 & 25 & I36 & $I_{4}$ & 82 & $\mathrm{I}_{4}$ & 109 & $\mathrm{NE} \times \mathrm{E}$ \\
\hline $\mathrm{T}_{\mathrm{M}}$ & 108 & 28 & 146 & 15 & II 2 & $2 I$ & I 22 & SSW \\
\hline
\end{tabular}

In summary it may be said that the observations strongly indicate that the layer of frictional influence reaches a height directly proportional to the wind speed at anemometer level, provided the wind has been uniform for a reasonable length of time, and provided other factors do not reach an unusual magnitude. Furthermore the proportionality factor gives values of $z_{0}$ which agree in a general way with the roughness of the ground, if the ratio $(s)$ between $z_{0}$ and the average height of the roughness elements is of the order of magnitude $\mathrm{I} / 30$. 


\section{Second Approximation}

Earlier we have referred to the restriction introduced by the assumption that the stress remains constant within the boundary layer $H$. We shall now attempt to estimate the error thus introduced. To do so we make use of the general equations of motion in the form $\left(\mathrm{I}_{4}\right)$, the gradient wind direction coinciding with the positive real $(x)$ axis and the imaginary $(y)$ axis pointing towards the low pressure. We introduce the angle $\varphi$ between the wind and the gradient wind and the angle $\psi$ between the vector of shear and the gradient wind. The equation may then be written in the form

$$
-\rho f i\left(W e^{i \varphi}-U_{\vartheta}\right)+\frac{\partial}{\partial z}\left(\tau e^{i \psi}\right)=0 .
$$

If one carries out the differentiation in the second term and makes use of the fact that $\varphi=\psi$ near the ground, it follows that

$$
-\rho f i\left(W-U_{g} \cos \varphi+i U_{c} \sin \varphi\right)+\frac{\partial \tau}{\partial z}+i \tau \frac{\partial \psi}{\partial z}=0
$$

near the ground. Separating real and imaginary parts we obtain two equations, viz.

$$
\begin{aligned}
\frac{\partial \tau}{\partial z} & =-\rho f U_{g} \sin \varphi \\
\tau \frac{\partial \psi}{\partial z} & =\rho f\left(W-U_{\imath} \cos \varphi\right) .
\end{aligned}
$$

The first of these equations gives the variation of $\tau$ with elevation, the second gives the rate of rotation of the stress vector in the same layer.

If we now make use of

$$
\tau_{H}=\frac{9}{4} \rho k^{2} U_{g}^{2} \sin ^{2} \varphi_{H},
$$

it follows that next to the ground

$$
\frac{\partial \tau}{\partial z}=-\frac{2 \rho f}{3 k} \sqrt{\frac{\tau}{\rho}}
$$

or, approximately,

$$
\sqrt{\frac{\tau}{\rho}}=\sqrt{\frac{\tau_{0}}{\rho}}-\frac{f}{3 k} z
$$

Thus small stresses decrease percentually more rapidly with elevation than stronger ones. To evaluate the result numerically, let us apply the result to open grass land $\left(z_{0}=3.2\right.$ $\mathrm{cm}$.), assuming a $10 \mathrm{~m}$. p.s. wind at $z_{a}=30 \mathrm{~m}$. In this case we have, roughly,

and

$$
\frac{\tau}{\rho}=2500
$$

$$
\frac{\partial}{\partial z}\left(\frac{\tau}{\rho}\right)=-0.05 \quad\left(f=10^{-4}\right)
$$


The decrease in $\tau / \rho$ over an elevation of roo m. amounts to 500 c.g.s. units or $20 \%$. If the wind at $30 \mathrm{~m}$. had been $5 \mathrm{~m}$.p.s. only, the decrease in $\tau / \rho$ over the same interval would have been 250 c.g.s. units or $40 \%$ of the total value of $\tau / \rho$.

The decrease in stress over the distance $H$ is given by

$$
\sqrt{\frac{\tau_{0}}{\rho}}-\sqrt{\frac{\tau_{H}}{\rho}}=\frac{f H}{3 k}=\frac{f h}{3 k_{0} \sqrt{2}}=\frac{k}{k_{0} \sqrt{2}} \cdot \sqrt{\frac{\tau_{H}}{\rho}}
$$

Thus

$$
\sqrt{\frac{\tau_{0}}{\rho}}=\mathrm{I} . \mathrm{I} 2 \sqrt{\frac{\tau_{H}}{\rho}}
$$

While these variations may seem fairly large, their effect on the velocity distribution is practically negligible. To establish this let us study Prandtl's expression for the stress in the form

$$
\sqrt{\frac{\tau}{\rho}}=\sqrt{\frac{\tau_{0}}{\rho}}-\frac{f}{3 k} z=l \frac{d W}{d z}
$$

or

$$
\frac{d W}{d z}=\frac{\mathrm{I}}{k_{0}\left(z+z_{0}\right)} \sqrt{\frac{\tau_{0}}{\rho}}-\frac{f}{3^{k k_{0}}} .
$$

Integration gives

$$
W=\frac{\mathrm{I}}{k_{0}} \sqrt{\frac{\tau_{0}}{\rho}} \ln \frac{z+z_{0}}{z_{0}}-\frac{f}{3 k k_{0}} z .
$$

The correction term amounts to at most 0.2 m.p.s. at an elevation of $100 \mathrm{~m}$. above the ground.

Next let us investigate the rotation of the stress vector within the same layer. The equation describing this rotation may be written in the form

$$
\frac{\tau}{\rho f} \frac{\partial \psi}{\partial z}=W-\cot \varphi \cdot U_{\sigma} \sin \varphi .
$$

Introducing in this equation the proper expressions for $W$ and $U_{v} \sin \varphi$ we find, in the vicinity of the ground,

$$
\frac{\tau}{\rho f} \frac{\partial \psi}{\partial z}=\frac{\mathrm{I}}{k_{0}} \sqrt{\frac{\tau_{0}}{\rho}} \ln \frac{z+z_{0}}{z_{0}}-\frac{2}{3 k} \sqrt{\frac{\tau_{0}}{\rho}} \cot \varphi .
$$

Thus

$$
\frac{\partial \psi}{\partial z}=\frac{f}{k_{0} \sqrt{\frac{\tau_{0}}{\rho}}}\left[\ln \frac{z+z_{0}}{z_{0}}-\frac{2 k_{0}}{3 k} \cot \varphi\right] .
$$


Integrating between the ground and the level $z$ we find

$$
\psi-\psi_{0}=\frac{f}{k_{0} \sqrt{\frac{\tau_{0}}{\rho}}}\left[\left(z+z_{0}\right) \ln \frac{z+z_{0}}{z_{0}}-z-\frac{2 k_{0}}{3 k} \cot \varphi \cdot z\right]
$$

or, in view of the smallness of $z_{0}$,

$$
\psi-\psi_{0}=\frac{f z}{k_{0} \sqrt{\frac{\tau_{0}}{\rho}}}\left[\ln \frac{z}{z_{0}}-\mathrm{I}-\frac{2 k_{0}}{3 k} \cot \varphi\right] .
$$

Thus the stronger stresses rotate less rapidly with elevation than the weaker ones.

Assuming again open grassland $\left(z_{0}=3.2 \mathrm{~cm}.\right)$, middle latitudes $\left(f=1 \mathrm{O}^{-4}\right)$ and a wind at $30 \mathrm{~m}$. of IO m.p.s. $\left(\tau_{0} / \rho=2500\right)$ we find, for the rotation over intervals of $50 \mathrm{~m}$. and IOO m.,

$$
\psi_{50}-\psi_{0}=- \text { O.II } 5, \quad \psi_{100}-\psi_{0}=-0.193
$$

or

$$
\psi_{0}-\psi_{50}=6.6^{\circ}, \quad \psi_{0}-\psi_{100}=\mathrm{II}^{\circ} .
$$

If in the same fashion we calculate the rotation between $z=0$ and $z=H$, we find, by use of (22), (25) and (3ob), that

$$
\psi_{0}-\psi_{H}=\frac{k}{k_{0} \sqrt{2}}\left(\sqrt{2}+\frac{3 k}{k_{0}}\right)=0.23=13^{\circ} .
$$

This strong rotation in $\psi$ is accompanied by a much smaller rotation in $\varphi$, the angle between the wind and the isobars. It is easy to see that next to the ground $d \varphi / d z$ must vanish. We have

$$
\frac{d \mathbf{W}}{d z}=\left(\frac{d W}{d z}+i W \frac{d \varphi}{d z}\right) e^{i \varphi}=C e^{i \psi}, \quad(\mathbf{W}=\text { wind vector })
$$

where $C$ represents the magnitude of the shearing vector. Since $\varphi_{0}=\psi_{0}$ it follows that

$$
\frac{d W}{d z}+i W \frac{d \varphi}{d z}=C
$$

or

$$
\frac{d \varphi}{d z}=\mathrm{o}
$$

In order to refine the solution with which we have been working it is evidently necessary to relinquish the condition that wind direction and stress direction agree at the level $z=H$.

From (A VII) and (A I 45) it follows that

$$
\sqrt{\frac{\tau_{H}}{\rho}}=\frac{f h}{3 k}
$$


VOL. III, NO. 3. FRICTIONAL INFLUENCE IN WIND AND OCEAN CURRENTS

Introducing the symbol $\Delta$ for the angle between $\varphi_{H}$ and $\psi_{H}$, that is,

$$
\Delta=\varphi_{H}-\psi_{H},
$$

one finds, from (A I $5 \mathrm{r}),\left(\mathrm{A}_{\mathrm{I}} 6_{4}\right)$ and $\left(\mathrm{A}_{\mathrm{I}} 6_{5}\right)$,

$$
W_{H}=U_{g} \cos \varphi_{H}-\frac{f h \sqrt{2}}{3 \sqrt{3} k^{2}} \cos (\Delta+\beta)
$$

and

$$
\circ=-U_{g} \sin \varphi_{H}+\frac{f h \sqrt{2}}{3 \sqrt{3} k^{2}} \sin (\Delta+\beta)
$$

Thus

$$
W_{H}=U_{o}\left[\cos \varphi_{H}-\cot (\Delta+\beta) \sin \varphi_{H}\right]
$$

and

$$
h=\frac{9 k^{2}}{2 f} U_{g} \sin \varphi_{H} \cdot \frac{\sin \beta}{\sin (\Delta+\beta)} .
$$

We have already found that

$$
\Delta=\frac{k}{k_{0} \sqrt{2}}\left(\sqrt{2}+\frac{3^{k}}{k_{0}}\right)=0.23=13^{\circ} .
$$
tain

Fitting together the two modified solutions for the upper and the lower layers we ob-

$$
\frac{\mathrm{I}}{k_{0}} \sqrt{\frac{\overline{\tau_{0}}}{\rho}} \ln \frac{H+z_{0}}{z_{0}}-\frac{f H}{3 k k_{0}}=U_{s}\left[\cos \varphi_{H}-\cot (\Delta+\beta) \sin \varphi_{H}\right]
$$

or

$$
\frac{\mathrm{I}}{k_{0}} \sqrt{\frac{\overline{\tau_{H}}}{\rho}}\left(\mathrm{I}+\frac{k}{k_{0} \sqrt{2}}\right) \ln \frac{H+z_{0}}{z_{0}}-\frac{f h}{3 k_{0}^{2} \sqrt{2}}=U_{0}\left[\cos \varphi_{H}-\cot (\Delta+\beta) \sin \varphi_{H}\right] .
$$

After some substitutions this equation takes the form

$$
\left(\mathrm{I}+\frac{k}{k_{0} \sqrt{2}}\right) \ln \frac{H+z_{0}}{z_{0}}=\frac{k}{k_{0} \sqrt{2}}+\frac{2 k_{0}}{3 k} \frac{\sin (\Delta+\beta)}{\sin \beta}\left[\cot \varphi_{H}-\cot (\Delta+\beta)\right] .
$$

Additional substitutions give, for the relation between $\varphi_{H}$ and $N$,

$$
\begin{aligned}
& \ln \left(\frac{9 k^{3}}{2 \sqrt{2} k_{0}} \frac{\sin \beta}{\sin (\Delta+\beta)} \sin \varphi_{H} \cdot N\right) \\
& \quad=\frac{k}{k_{0} \sqrt{2}+k}+\frac{2 k_{0}}{3 k\left(\mathrm{I}+\frac{k}{k_{0} \sqrt{2}}\right)} \frac{\sin (\Delta+\beta)}{\sin \beta}\left[\cot \varphi_{H}-\cot (\Delta+\beta)\right. \text { ।. }
\end{aligned}
$$


A graphical representation of this solution is given in Fig. 7 through the dotted line marked second approximation. As might be expected this second approximation gives slightly higher $\varphi$-values for given values of $N$ but the difference is only about $3^{\circ}$ in the region of $N=10^{6}$. It does become significant for very low values of $N$.

Considering the small variations in $W$ and $\varphi$ between the first and the second approximation it seems justifiable to accept the first approximation as a sufficiently accurate basis for most investigations.

\section{INFLUENCE OF STABILITY}

\section{REVIEW}

Various writers have attempted to determine the relation between stability and eddyviscosity. While it is generally recognized that increased stability will reduce the stirring in air and water, a satisfactory theory for the relation between the two has not yet been established. From an analysis of temperature and wind data obtained at different levels along the Eiffel tower, Exner ${ }^{24}$ attempted to show that the eddy-viscosity coefficient is inversely proportional to $\alpha_{\rho}-\alpha$, where $\alpha_{\rho}$ represents the lapse rate of an atmosphere of constant density $\left(\alpha_{p}=m g / R=3.4^{\circ} \mathrm{C} / 100 \mathrm{~m}\right.$.). Ertel ${ }^{25}$ has offered theoretical support for Exner's conclusion. In view of the importance of the problem it seems advisable to study the methods used by these authors before making another attack on the problem.

Making use of a simplified form of the equations of motion and neglecting the deflecting force, Exner obtains for the tangential frictional force the expression

$$
\tau=\eta \frac{d W}{d z}=A z+B,(A \text { and } B \text { constants, } z \text { height above the ground })
$$

in which, for the sake of convenience, the constant term is dropped so that the formula reduces to

$$
\frac{\tau}{\eta}=\frac{d W}{d z}=\frac{A z}{\mu}
$$

Assuming that the eddy viscosity can be written in the form

$$
\eta=\frac{c}{K+\frac{d T}{d z}}
$$

where $T$ represents temperature and the constant $K>g / c_{p}$, Exner finds

$$
\frac{d W}{d z}=\frac{A}{c}\left(K+\frac{d T}{d z}\right) \cdot z \text {. }
$$

From Angot's values for the mean monthly temperature distribution along the Eiffel tower (measured at four levels) $d T / d z$ is determined. The constant $A / c$ is assumed to be proportional to the mean monthly wind velocity and to the mean monthly temperature at the top of the Eiffel tower. It is then possible to integrate the expression for $d W / d z$. 
The result is

$$
W_{z}-W_{0}=\frac{A}{c}\left[K \frac{z^{2}}{2}+\int_{0}^{z} z \frac{d T}{d z} d z\right]
$$

From this equation $K$ is computed by comparing observed and theoretical wind velocity differences between the top of the Eiffel tower and the surface anemometer.

Exner's values for $K$ range between $2.2 \cdot 1 \mathrm{O}^{-4}$ and $4 \cdot \mathrm{I} 3 \cdot 1 \mathrm{O}^{-4}$ with a mean value of $3.2 \mathrm{I} \cdot \mathrm{IO}^{-4}$. This mean value comes so close to the theoretical figure

$$
\frac{m g}{R}=3 \cdot 4 \cdot 10^{-4}
$$

that Exner feels justified in assuming that

$$
\eta=\frac{c}{\frac{m g}{R}+\frac{d T}{d z}}
$$

There are two weak points in Exner's analysis. In the first place expression (73b) for the stress is entirely at variance with available evidence. Solberg ${ }^{8}$ has pointed out that the frictional drag remains practically constant for the first hundred or two hundred meters. If the frictional drag vanishes at the ground, as implied by $(73 \mathrm{~b})$, there are no forces that could possibly lead to the development of strong internal stresses some distance from the ground.

Prandtl's well established theory for the velocity distribution in the vicinity of a rigid, rough boundary, a theory which we have found to apply also to atmospheric phenomena, implies that the motion near the ground must be controlled essentially by frictional drag. This means that in the expression (73a) for the stress the constant term B dominates over the small linear term $A z$.

Assuming then that

$$
\tau=\eta \frac{d W}{d z}=B(\text { constant })
$$

it is readily seen that the assumed expression for $\mu$ leads to

$$
\frac{d W}{d z}=\frac{B}{c} \cdot\left(K+\frac{d T}{d z}\right)
$$

which may be integrated to give

$$
W_{2}-W_{1}=\frac{B}{c}\left[K\left(z_{2}-z_{1}\right)+T_{2}-T_{1}\right]
$$

Thus, under rational assumptions regarding the stress, Exner's theory leads to the simple result that the velocity difference between two levels must be a linear function of the temperature difference between the same levels. Heywood ${ }^{26}$ in an important investigation discussed below, shows through simultaneous temperature and wind velocity records, that the relation is much more complex. The wind velocity difference $\left(W_{2}-W_{1}\right)$ 
grows at first rapidly, then more slowly with $T_{2}-T_{1}$ until finally an asymptotic value seems to be reached beyond which an increase in the intensity of the inversion does not materially change the difference in wind velocity.

A second weak point in Exner's method of treating the observations is to be found in the assumed form of the eddy-viscosity, which makes it independent of elevation. This was remedied by Ertel, whose analysis we shall now consider. It may be summarized as follows:

If $v_{z}$ represents the turbulent vertical velocity component, the net vertical transport $M$ of air through a small horizontal element of area $d F$ during the time $t$ is given by

$$
M=d F \int_{0}^{t} \rho v_{z} d t
$$

On the average this transport must vanish. Thus

$$
-\overline{\rho v}_{z}=0 \text {, }
$$

where the bar represents a time average. According to Ertel, the ascending parcels of air will have a higher density, the descending parcels a lower density than the average prevailing at $d F$. Thus, if $\overline{\rho v_{z}}$ vanishes, this must mean that

$$
\bar{v}_{z}<0 \text {. }
$$

The conclusion is hardly justified. Taking into consideration the compressibility of the atmosphere and the presumably adiabatic changes taking place in the moving elements it is seen that Ertel's conclusion holds for an atmosphere in which the vertical temperature lapse rate is less than the dry-adiabatic. For an unstable atmosphere $\left(\frac{d T}{d z}<-\frac{g}{c_{p}}\right)$ the reverse would hold, thus, in such an atmosphere

$$
\bar{v}_{z}>0 \text {. }
$$

Consequently $\bar{v}_{z}$, regarded as a function of the lapse rate, changes sign for

$$
\frac{d T}{d z}=-\frac{g}{c_{p}}
$$

and one may therefore conclude that it contains a factor $d T / d z+g / c_{p}$.

Ertel then calculates the density of an element at $d F$ which has arrived from a layer located a distance $\xi$ below $d F$. If $\bar{\rho}$ represents the mean density at $d F$, this moving element, according to Ertel, arrives at $d F$ with the density

$$
\rho=\bar{\rho}-\frac{\partial \bar{\rho}}{\partial z} \xi=\bar{\rho}-\frac{\partial \ln \bar{\rho}}{\partial z} \cdot \bar{\rho} \xi=\bar{\rho}+\frac{\bar{\rho} \xi}{T}\left[\frac{m g}{R}+\frac{d T}{d z}\right] .
$$

Multiplying this equation with $v_{z}$ and forming a time average one obtains, in view of the fact that $\frac{g}{\rho v_{z}}$ disappears,

$$
\eta \equiv \bar{\rho} \cdot \overline{\xi v_{z}}=-\frac{\bar{\rho} T \overline{v_{z}}}{\frac{m g}{R}+\frac{d T}{d z}}
$$


showing that $\eta$ is inversely proportional to the difference between the constant density lapse rate and the actual lapse rate. The formula is more general than the one obtained by Exner, since the numerator now may be any function of elevation.

Unfortunately, Ertel's calculation of the density at $d F$ again suffers from his omission to take compressibility and adiabatic changes into account. Ertel's expression implies that the moving element carries along, unchanged, its original density. Actually,

$$
\rho=\bar{\rho}_{z-\xi}+\delta_{i} \rho
$$

where $\delta_{i} \rho$ represents the adiabatic change in density of the moving element during its displacement $\xi$ upward. This second term has the value

$$
\delta_{i} \rho=-\frac{c_{v}}{c_{p}} \frac{\rho}{T} \frac{m g}{R} \xi .
$$

Neglecting small terms of higher order this gives

$$
\rho=\bar{\rho}+\frac{\bar{\rho}}{T} \xi\left[\frac{m g}{R}+\frac{d T}{d z}-\frac{c_{v}}{c_{p}} \frac{m g}{R}\right]=\bar{\rho}+\frac{\bar{\rho}}{T} \xi\left[\frac{g}{c_{p}}+\frac{d T}{d z}\right] .
$$

Consequently, if compressibility is taken into account,

$$
\eta \equiv \bar{\rho} \cdot \overline{\xi v_{z}}=-\frac{\bar{\rho} T \overline{v_{z}}}{\frac{g}{c_{p}}+\frac{d T}{d z}} .
$$

This expression, which follows from Ertel's theory if the adiabatic density changes of the moving elements are taken into consideration, would seem to indicate that the eddyviscosity coefficient approaches infinity already when the lapse rate reaches the adiabatic, a result which certainly does not agree with established facts. However, it was pointed out above that $\bar{v}_{z}$ also must contain the factor $g / c_{p}+d T / d z$; the two factors may cancel each other and one is therefore left in doubt concerning the relation between stability and eddy-viscosity.

\section{Stability in the Boundary Layer}

Let us now consider the effect of stability in the surface layers, where the motion is simple in character and the stress is approximately independent of elevation. It is well known that a stable stratification tends to dampen the turbulent vertical movements of the air. At a certain elevation $z$ above the ground we assume the mixing length to have the value $l_{s}$, whereas its value in the absence of stability would be

$$
l=k_{0}\left(z+z_{0}\right) \text {. }
$$

If the prevailing rate of shear is $C_{s}$, it follows that the turbulent kinetic energy per unit mass, $\frac{1}{2} \overline{\left(u^{\prime 2}\right.}+\overline{v^{\prime 2}}+\overline{\left.w^{\prime 2}\right)}$, must be proportional to $l_{s}^{2} C_{s}^{2}$. If the rate of shear were the same but the stability were zero, the turbulent kinetic energy would be proportional to $l^{2} C_{s}{ }^{2}$. We assume that the difference now occurs in the form of potential energy, the turbulent elements at every instant having a density differing from the one prevailing in the 
surroundings. This potential energy must be proportional to $(g / \theta)(d \theta / d z) l_{s}{ }^{2}$, where $\theta$ represents the potential temperature and $g$ the acceleration of gravity. Thus

$$
l^{2} C_{s}^{2}=l_{s}^{2} C_{s}^{2}+\frac{\beta g}{\theta} \frac{d \theta}{d z} l_{s}^{2}
$$

where $\beta$ is a proportionality factor as yet undetermined. It follows from the above equation that

$$
l_{s}=\frac{l}{\sqrt{\mathrm{I}+\frac{\kappa^{2}}{C_{s}^{2}}}} \quad\left(\kappa^{2}=\frac{\beta g}{\theta} \frac{d \theta}{d z}\right)
$$

Now let us compare a homogeneous and a stratified medium moving under the influence of the same stress. The rates of shear will vary and the two values will be designated by $C_{s}$ and $C$. Assuming the expression (3) to be valid also in the case of a stratified medium, we obtain

$$
\sqrt{\frac{\tau}{\rho}}=l_{s} C_{s}=l C
$$

Since equation ( 92 ) for $l$ is independent of the rate of shear it is permissible to combine (94) with (95). The result is

$$
C_{s}=C \sqrt{\mathrm{I}+\frac{\kappa^{2}}{C_{s}^{2}}}
$$

In case of an incompressible medium (water) one has

$$
\kappa^{2}=-\frac{\beta g}{\rho} \frac{d \rho}{d z}
$$

Solving (96) one obtains

$$
\frac{C_{s}}{C} \equiv u=\sqrt{\frac{1}{2}+\frac{1}{2} \sqrt{\mathrm{I}+\frac{4^{\kappa^{2}}}{C^{2}}}}
$$

In order to check the preceeding reasoning it is necessary to draw the ultimate consequences of the formulae presented above and compare them with available observation material.

From (98) combined with

$$
C=\frac{\mathrm{I}}{k_{0}\left(z+z_{0}\right)} \sqrt{\frac{\tau}{\rho}},
$$

it follows that

$$
\frac{d W_{s}}{d z}=\frac{\mathrm{I}}{k_{0}\left(z+z_{0}\right)} \sqrt{\frac{\tau}{\rho}} \sqrt{\frac{1}{2}+\frac{1}{2} \sqrt{\mathrm{I}+\frac{4 \kappa^{2}\left(z+z_{0}\right)^{2}}{\left(\frac{\mathrm{I}}{k_{0}} \sqrt{\frac{\tau}{\rho}}\right)^{2}}}} .
$$


VOL. III, NO. 3. FRICTIONAL INFLUENCE IN WIND AND OCEAN CURRENTS

Integration of (99) between the two levels $z_{2}$ and $z_{1}$ gives

$$
W_{s 2}-W_{s 1}=\frac{\mathrm{I}}{k_{0}} \sqrt{\frac{\tau}{\rho}}\left[2\left(u_{2}-u_{1}\right)-\frac{1}{2} \ln \frac{u_{2}+\mathrm{I}}{u_{2}-\mathrm{I}} \frac{u_{1}-\mathrm{I}}{u_{1}+\mathrm{I}}\right],
$$

where $W_{s}$ represents the wind velocity in the stable case as distinguished from the adiabatic wind velocity $W_{a d}$.

If we integrate between the ground and the level $z$, we have, because of the smallness of $z_{0}$,

( $\mathrm{I} O \mathrm{I})$

$$
u_{0}=I+\frac{1}{2} \frac{\kappa^{2} z_{0}^{2}}{\left(\frac{\mathrm{I}}{k_{0}} \sqrt{\frac{\tau}{\rho}}\right)^{2}}
$$

which differs only slightly from I. Thus

$$
W_{s}=\frac{\mathrm{I}}{k_{0}} \sqrt{\frac{\tau}{\rho}}\left[2(u-\mathrm{I})-\frac{1}{2} \ln \frac{u+\mathrm{I}}{u-\mathrm{I}}+\ln \frac{\frac{2}{k_{0}} \sqrt{\frac{\tau}{\rho}}}{\kappa z_{0}}\right]
$$

or,

$$
\begin{aligned}
W_{s} & =\frac{\mathrm{I}}{k_{0}} \sqrt{\frac{\tau}{\rho}}\left[\ln \frac{z+z_{0}}{z_{0}}+2(u-\mathrm{I})-\ln \frac{u(u+\mathrm{I})}{2}\right] \\
& =W_{a d}+\frac{\mathrm{I}}{k_{0}} \sqrt{\frac{\tau}{\rho}}\left[2(u-\mathrm{I})-\ln \frac{u(u+\mathrm{I})}{2}\right] .
\end{aligned}
$$

The expression

$$
\frac{W_{s}-W_{n d}}{\frac{\mathrm{I}}{k_{0}} \sqrt{\frac{\tau}{\rho}}}=f_{1}(u)=f_{2}\left(\frac{\kappa z}{\frac{\mathrm{I}}{k_{0}} \sqrt{\frac{\tau}{\rho}}}\right)
$$

is represented graphically in Fig. I7.

It is easily shown that ( $\left.\mathrm{IO}_{3}\right)$ changes into the previously given solution for a homogeneous medium for $\kappa^{2} \rightarrow 0$. In this case $u$ approaches I and the term $2(u-I)-\ln [u(u+1) / 2]$ approaches zero. Since $u-I$ goes towards zero with decreasing values of $z$, it follows that $\left(\mathrm{IO}_{4}\right)$

$$
W_{s}-W_{a d} \rightarrow 0
$$

independent of the value of $\kappa^{2}$. 
The eddy-viscosity coefficient, given by
(I05a)
$\eta_{s}=\rho l_{s}^{2} C_{s}$,

will now have the value

(105b)

$$
\eta_{s}=\frac{\eta}{u}=\frac{\rho k_{0}\left(z+z_{0}\right) \sqrt{\frac{\tau}{\rho}}}{\sqrt{\frac{1}{2}+\frac{1}{2} \sqrt{\mathrm{I}+\frac{4^{\kappa^{2} z^{2}}}{\left(\frac{\mathrm{I}}{k_{0}} \sqrt{\left.\frac{\tau}{\rho}\right)^{2}}\right.}}}} .
$$

It will therefore increase upward at a slower rate than in a homogeneous medium.

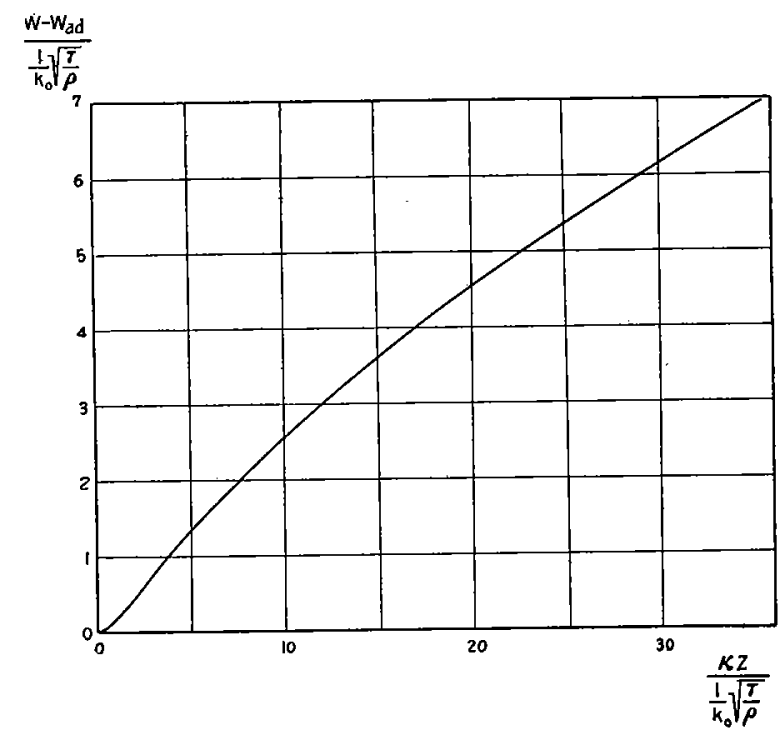

Fig. I7.-Theoretical difference between stable and adiabatic wind velocity for different values of stress, stability and distance from the ground.

For large values of the stability one has, with increasing degree of accuracy,

$$
u \rightarrow \frac{\sqrt[4]{\kappa^{2}}}{\sqrt{\frac{1}{k_{0}} \sqrt{\frac{\tau}{\rho}}}} \sqrt{z}
$$

and consequently

$$
W_{s 2}-W_{s 1} \rightarrow 2 \sqrt{\frac{\kappa}{k_{0}} \sqrt{\frac{\bar{\tau}}{\rho}}}\left(\sqrt{z_{2}}-\sqrt{z_{1}}\right) .
$$

This formula indicates that as the stability increases the difference in wind velocity between two different levels near the surface of the earth tends to become proportional to the 
difference between the square roots of the elevations. This theoretical result is fairly well confirmed by the observations discussed below.

Heywood, ${ }^{26}$ in an experimental investigation at Leafield in England, measured the wind velocity at two levels $(95 \mathrm{~m}$. and $13 \mathrm{~m}$.) and determined the differences in velocity as a function of temperature gradient and wind velocity at the upper anemometer. The temperature gradient was determined by readings at $87 \mathrm{~m}$. and $13 \mathrm{~m}$. Heywood's data have been used for a check of the theory outlined above. In order that the preceeding theory may be applied, one must assume that the stress $\tau$ remains fairly constant up to a level of $95 \mathrm{~m}$. above the ground; this assumption is not correct, particularly not in case of light winds. The error thus introduced will be analyzed later.

The first step in the theoretical evaluation of Heywood's data consists in the determination of $z_{n}$ for Leafield.

In Fig. 2 and Table III of Heywood's paper hourly averages for wind velocity difference between the two levels is tabulated for different wind velocities and for summer and winter separately. The curves for the summer season show a rapid decrease in the vertical wind gradient during the morning hours and an equally abrupt increase in the evening. Between IO A.M. and 4 P.M. the wind gradient is practically constant. It is reasonable to assume that during this period the temperature lapse rate must be approximately adiabatic. If we follow Heywood's designations and represent by $V$ the wind velocity at $95 \mathrm{~m}$. and by $v$ the velocity at $\mathrm{I} 3 \mathrm{~m}$., we obtain the following mean values for the period IO A.M. to 4 P.M., inclusive:

TABLE 19

\begin{tabular}{|c|c|c|c|c|}
\hline Group & $V$ m.p.s. & $v$ m.p.s. & $(V-v)$ m.p.s. & $z_{0} \mathrm{~cm}$ \\
\hline $\begin{array}{l}\text { Strong } \\
\text { Moderate } \\
\text { Light }\end{array}$ & $\begin{array}{r}\mathrm{II} .45 \\
7.57 \\
4.38\end{array}$ & $\begin{array}{l}8.4 \mathrm{I} \\
5.52 \\
2.95\end{array}$ & $\begin{array}{l}3.04 \\
2.02 \\
1.43\end{array}$ & $\begin{array}{r}5 \cdot 3 \\
5 \cdot 5 \\
2 I .5\end{array}$ \\
\hline
\end{tabular}

From the data in this table we can determine $z_{0}$ by means of the relation

$$
\frac{\log \frac{9500}{1300}}{\log \frac{9500}{z_{0}}}=\frac{V-v}{V}
$$

The results are given in the last column of the table. The light winds are too erratic to be given full weight. We have chosen for $z_{0}$ a value of $6 \mathrm{~cm}$., corresponding to a roughness amplitude of about $1.8 \mathrm{~m}$.; this agrees with the fact that in two directions (SW and NE) the wind has to pass over brush land.

The curves for the winter season indicate reasonably constant values of $V-v$ between noon and 2 P.M. The corresponding values are given below.

TABLE 20

\begin{tabular}{lrrrr}
\hline Group & $V$ m.p.s. & $v$ m.p.s. & $(V-v)$ m.p.s. & $z_{0} \mathrm{~cm}$. \\
\hline Strong & I2.12 & 8.47 & 3.65 & I2.9 \\
Moderate & 6.93 & 4.82 & $2 . \mathrm{II}$ & $\mathrm{I3} .8$ \\
Light & 4.82 & 2.60 & $\mathrm{I.36}$ & 8.3 \\
\hline
\end{tabular}


The resulting $z_{0}$-values are considerably higher than those found from the summer data. However, because of the uncertainty that the winter observations really are associated with adiabatic lapse rates they have not been considered in the determination of $z_{0}$ for Leafield.

Heywood's three wind groups are defined in the following way:

$V \geqq 9$ m.p.s. for strong winds

9 m.p.s. $>V>6$ m.p.s. for moderate winds

6 m.p.s. $\geqq V \geqq 3$ m.p.s. for light winds

In winter time it is quite possible to have a wind velocity of 9 m.p.s. at $95 \mathrm{~m}$. with á stable lapse rate or even an inversion at lower levels. For our purposes it would have been better if the separation of the observational material in three groups had been based on the wind velocities recorded at the lower anemometer. Before proceeding it is worth while to note that Heywood's curves for the winter season show practically constant values of $V-v$ between 6 P.M. and 7 A.M. for strong and moderate winds. During the summer, reasonably constant values prevail between 9 P.M. and 5 A.M. During both seasons the light winds behave in a more erratic fashion. One may perhaps assume that the night inversions at Leafield during the hours listed above are strong enough for the vertical wind gradient to approach its asymtotic value. To test this assumption I have tabulated below the ratio $(V-v) / V$. It would appear that for light and moderate winds this ratio tends to approach its theoretical limit of 0.63 .

TABLE 21

\begin{tabular}{llrrr}
\hline & Group & $V$ & $V-v$ & $\frac{V-v}{V}$ \\
\hline Winter & Strong & 11.62 & 4.72 & 0.41 \\
6 P.M.-7 A.M. & Moderate & 7.76 & 3.78 & 0.49 \\
Summer & Light & 5.28 & 2.85 & 0.54 \\
9 P.M.-5 A.M. & Strong & 9.76 & 4.18 & 0.43 \\
Moderate & 7.54 & 3.98 & 0.53 \\
\hline
\end{tabular}

Expression (I06) shows that $u$ approaches its asymptotic value $\sqrt{\frac{\kappa z}{\frac{\mathrm{I}}{k_{0}} \sqrt{\frac{\tau}{\rho}}}}$ more

rapidly for small stresses than for large ones, i.e. more quickly for light than for strong winds. Furthermore, $\kappa^{2}$ is probably greatest for light winds. This would seem to explain the tendency for $(V-v) / V$ to increase with decreasing winds. Until more complete temperature data are available it is impossible to test this explanation. It is worth pointing out that none of the $(V-v) / V$ values exceeds the theoretical limit of 0.63 .

From this preliminary analysis it would seem that the air motion in the lowest layer of the atmosphere $(0-100 \mathrm{~m}$.) sways between two regîmes-one controlled by the mechanical turbulence originating at the ground and characterized by the roughness of the station in question, the other controlled by the stable stratification and obeying the simple asymptotic law derived on page 50. Heywood's curves for $V-v$ indicate that the wind most of the time follows the one or the other of these two regimes, and that the transition periods are of relatively short duration, 5 A.M. to IO A.M. and 4 P.M. to 9 P.M. in the summer, 7 A.M. to noon and 2 P.M. to 6 P.M. in the winter. 
The value of $z_{0}$ being known it is possible to calculate the theoretical value of $V$ and $v$ for different values of stress $(\sqrt{\tau / \rho})$, and stability $\left(\kappa^{2}\right)$. The values for $V-v$ are independent of $z_{0}$ and depend on $\sqrt{\tau / \rho}$ and $\kappa^{2}$ only. In Fig. 18 the results are plotted in the form of a group of curves, each characterized by a constant value of $\kappa^{2}$ and giving $V-v$ as a function of $V$. This diagram is strikingly similar to Heywood's Fig. 5, which gives $V-v$ as a function of $V$ for different values of $T_{78}-T_{13}{ }^{*}$ In order to determine the

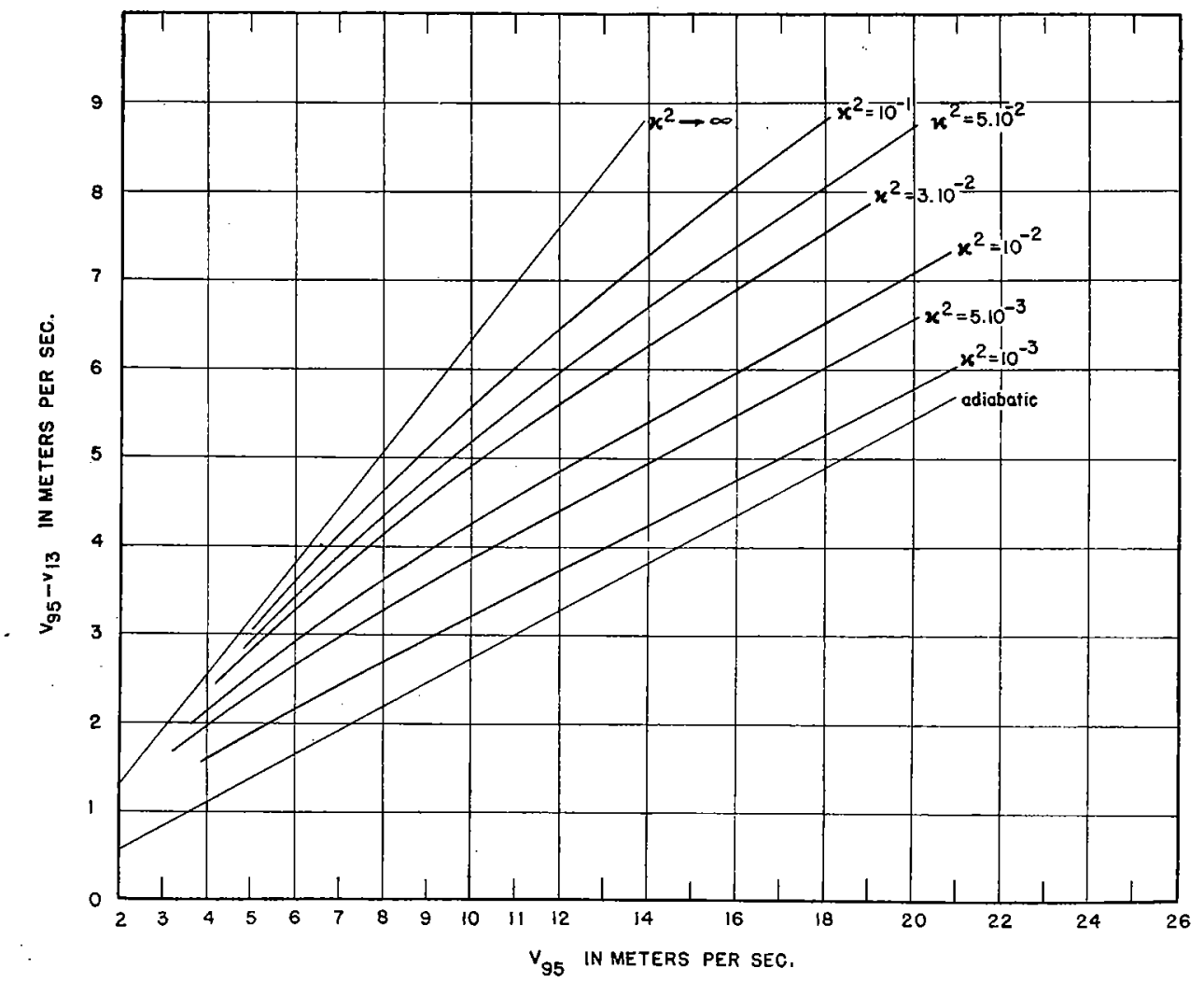

FIG. 18.-Theoretical value of the wind gradient at Leafield for different values of stability and wind velocity.

constant $\beta$ in the definition of the stability parameter (94), a new diagram has been prepared with logarithmic scale for $\kappa^{2}$ and linear scale for $V-v$. In this diagram (Fig. I9) and on the appropriate $V$-curves, Heywood's values for $V$-v were entered. To each point thus obtained corresponds a definite value of $T_{87}-T_{13}$ and also a definite value of $\kappa^{2}$. In case of perfect agreement between theory and observations all points with the same value of $T_{87}-T_{13}$ must fall on a horizontal line (constant $\kappa^{2}$ ). The deviations are fairly large, particularly on the curve $V=5$ m.p.s., i.e. for light winds. If these points be excluded the diagram indicates a mean value of $\beta$ in the vicinity of 40.

Having arrived at a preliminary value for $\beta$ we can now prepare theoretical curves for $V-v$ as a function of $\Delta T$ and $V$, using Fig. I 8 and interpreting the $\kappa^{2}$-values in terms of $\Delta T$. Three such curves were prepared for $V=$ I I, 7.5 and 4.5 m.p.s., corresponding to Heywood's strong, moderate and light winds. The three curves are plotted in Fig. 20.

* Heywood's diagram is based on readings from the single month of March, I929. 
In the same diagram I have entered Heywood's curves $V-v$ for the three groups as functions of $\Delta T$ (Heywood, Fig. 3). These curves are based on the complete observation material for the period January-June 1929, and should therefore be more reliable than the curves in Heywood's Fig. 5. The agreement is satisfactory. Both theory and observations indicate a sudden increase in the wind gradient as the lapse rate changes from the adiabatic to a slightly stable value. A further increase of the stability influences the velocity distribution but slightly. This is particularly noticeable in the light wind group.

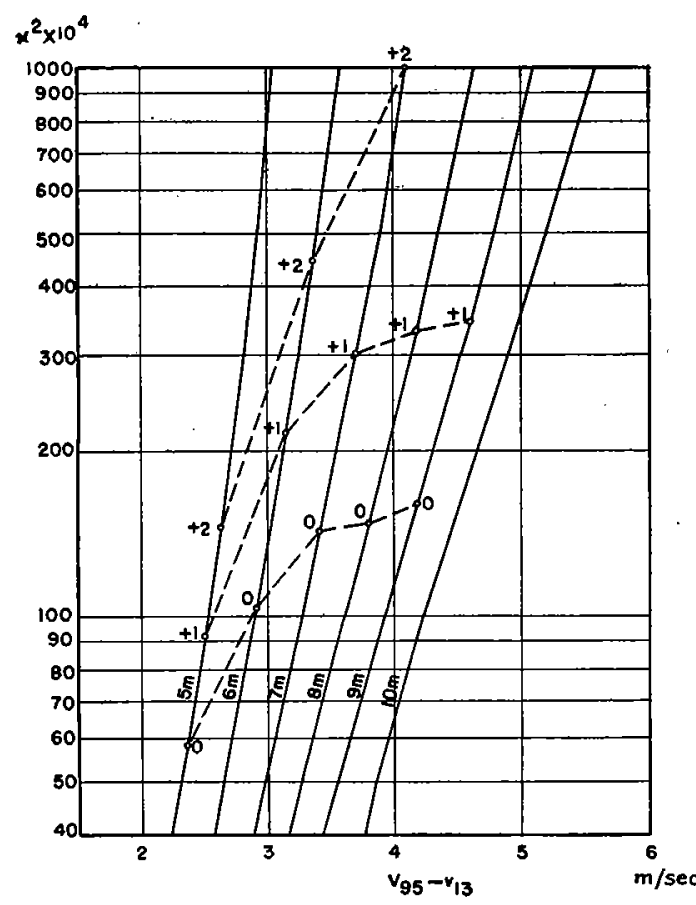

FIG. I9.-Graphical determination of $\beta$ from observed wind gradient over Leafield.

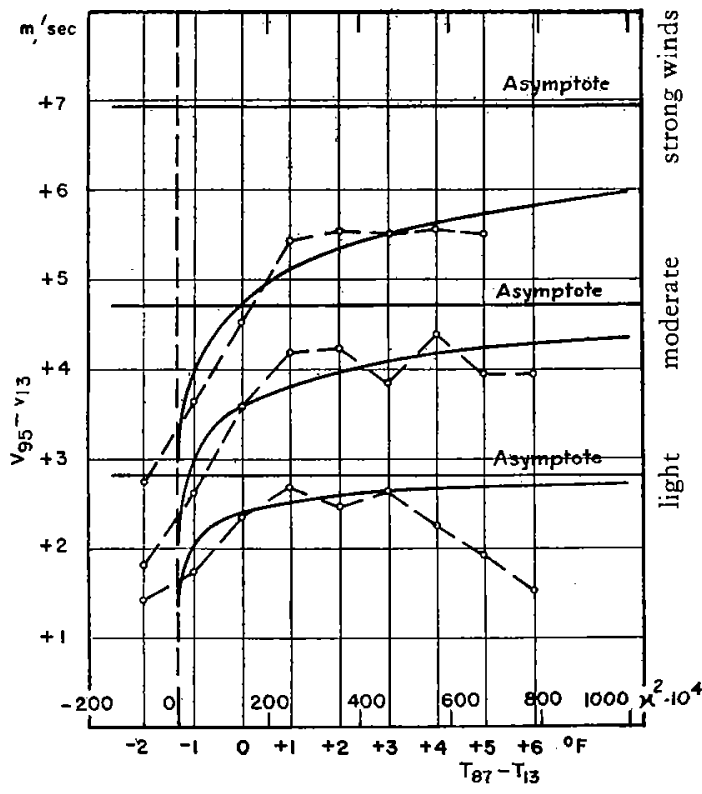

Fig. 20.- Comparison between observed and computed wind gradients over Leafield for different temperature gradients and wind velocities (theoretical values given by full lines, observations by broken lines).

In the discussion following Heywood's paper Durst points out that Heywood has excluded all cases where the wind recorded on the lower anemometer was less than 2 m.p.s. Observations at Cardington indicate that the vertical gradient of the wind velocity $\left(W_{50}-W_{13}\right)$ increases steadily with increasing stability, even for light winds. Durst is, therefore, inclined to doubt the reality of this particular phase of Heywood's result and attribute it to the method used in selecting the observations (exclusion of $V$-values $<3$ m.p.s., $v$-values $<2$ m.p.s.), which automatically eliminates large values of $V-v$ in the light wind group. However, it is difficult to see how Heywood's method of selection could produce an entirely fictitious maximum of $V-v$ in the light wind group. At this point it is sufficient to mention that the assumption of a constant stress up to $95 \mathrm{~m}$. certainly can not be valid for light winds in a highly stable atmosphere. It was brought out in the discussion of the second approximation that small stresses decrease more rapidly with elevation than large ones. This tendency, derived for an adiabatic atmosphere, is still more marked in a stable atmosphere. 
In general, it would seem that Heywood's data support the theory outlined above. It must be kept in mind that this agreement of three experimental and three theoretical curves is obtained with the aid of a single available constant, $\beta .^{*}$

It is possible to obtain a check on the preceding theory with the aid of Hellman's wind measurements at Nauen (our Table 3 )..$^{10}$

It was pointed out earlier that for high values of the stability the difference in wind velocity between two levels $\left(z_{2}\right.$ and $z_{1}$ ) tends to become proportional to $\sqrt{\overline{z_{2}}}-\sqrt{z_{1}}$. Working with data from three different levels one obtains

$$
\frac{W_{3}-W_{2}}{W_{2}-W_{1}} \rightarrow \frac{\sqrt{z_{3}}-\sqrt{z_{2}}}{\sqrt{z_{2}}-\sqrt{z_{1}}}
$$

which expression enables us to check the asymptotic solution derived above without requiring that $\tau$ or $\kappa^{2}$ be known. In the adiabatic case the corresponding ratio has the value

$$
\frac{W_{3}-W_{2}}{W_{2}-W_{1}}=\frac{\ln \frac{z_{3}}{z_{2}}}{\ln \frac{z_{2}}{z_{1}}}
$$

In the course of a twenty-four hour period the lapse rate tends to vary between its adiabatic value in the early afternoon and a highly stable value in the early morning and one should expect the ratio $\left(W_{3}-W_{2}\right) /\left(W_{2}-W_{1}\right)$ to vary accordingly. I have made use of Hellmann's data to check the theoretical result stated above. Setting $z_{3}=32 \mathrm{~m}$., $z_{2}=16 \mathrm{~m}$. and $z_{1}=2 \mathrm{~m}$., the two limiting values indicated by the theory are

$$
\frac{\sqrt{32}-\sqrt{16}}{\sqrt{16}-\sqrt{2}}=0.64, \frac{\log \frac{32}{16}}{\log \frac{16}{2}}=0.33 .
$$

* Durst has emphasized that observations at Cardington apparently contradict Heywood's conclusion regarding the variation of the vertical gradient of the wind with the vertical temperature distribution. From a study of frequency curves for the ratio of the wind velocity at the 150 foot level to the wind velocity at the 50 foot level for different values of the temperature lapse rate, Durst concludes that the ratio between the two wind velocities increases indefinitely with increasing stability. An inspection of Appendix V (Table C) in the Cardington report ${ }^{27}$ shows that the number of observations available in the high stability group is much smaller than in Heywood's case and the results correspondingly more uncertain. In particular, the principal group of observations (June, July and August, $22 \mathrm{~h}$ to $3 \mathrm{~h}, \mathrm{IO}-\mathrm{I}_{4} \mathrm{~m}$.p.h. at I 50 feet) strongly suggests the existence of a secondary frequency maximum for

separated from the main group by zero frequencies at

$$
\frac{W_{50}}{W_{13}} \geqq 2.0,
$$

$$
\frac{W_{50}}{W_{13}}=1.9 .
$$

It the observations pertaining to the secondary maximum are eliminated the remaining data approach, but do not exceed, the theoretical limit

$$
\frac{W_{50}}{W_{13}} \rightarrow \sqrt{3}=1.73 .
$$

It is hardly possible to explain the secondary maximum without a detailed analysis of the individual observations, but an inspection of the map of the Cardington area (Fig. 2 in the report) definitely suggests that sloping inversions between the two anemometer levels might easily develop, particularly for light NW winds. These sloping inversions may set up opposing horizontal pressure gradients which will lower or reverse the wind velocity at the 50 -foot level. 
In Figs. $2 \mathrm{Ia}$ and $2 \mathrm{Ib}$ graphical representations of the diurnal variation in $\left(W_{32}-W_{16}\right) /\left(W_{16}-W_{2}\right)$ are given. In Fig. 2 r a light and strong winds are treated separately and separate curves are drawn for the warm and the cold season. Fig. $2 \mathrm{Ib}$ gives the mean curves for one year for these two wind groups and also for the entire observation material (taken from Hellmann's Table 3). Both figures show that in case of strong winds the ratio $\left(W_{32}-W_{16}\right) /\left(W_{16}-W_{2}\right)$ oscillates within a comparatively narrow range, always

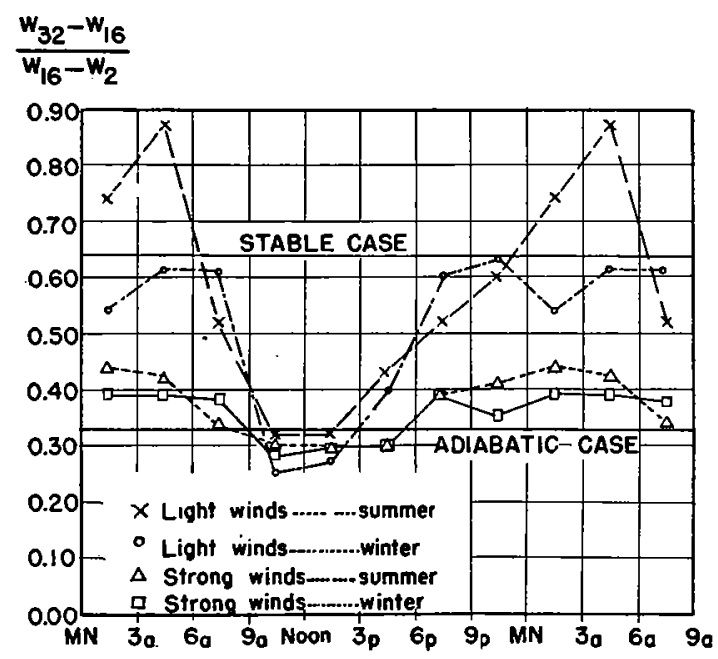

Fig. 2Ia.-Diurnal variation of the vertical wind distribution $\left(\frac{W_{32}-W_{16}}{W_{16}-W_{2}}\right)$ for different wind velocities and

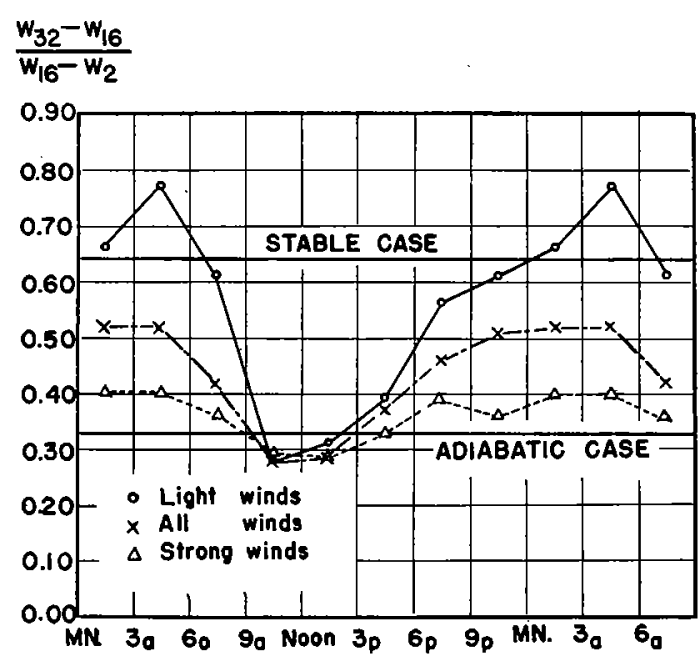

Fig. 2Ib.-Diurnal variation of the vertical wind distribution $\left(\frac{W_{32}-W_{16}}{W_{16}-W_{2}}\right)$ for different wind velocities (one year's mean, from Hellmann's data).

remaining fairly close to the adiabatic value of 0.33 . Since strong winds bring about intense mixing and thus prevent or retard the development of night inversions the result is hardly surprising. The light wind group for the cold season is characterized by a practically constant value of $\left(W_{32}-W_{16}\right) /\left(W_{16}-W_{2}\right)$ from 7:30 P.M. to 7:30 A.M. The numerical value of this constant is about 0.6 , in good agreement with the theoretical value, 0.64 . The light wind group for the warm season shows extremely high values of $\left(W_{32}-W_{16}\right) /\left(W_{16}-W_{2}\right)$ between midnight and 6 A.M.; it is possible that this part of the curve may be explained by vertical variations in the stability. In the absence of temperature gradient measurements it is impossible to analyze the curve in detail.

\section{Stability Within Entire Frictional Layer}

An inspection of the fundamental equations of the preceeding theory reveals that the characteristic difference between the adiabatic and the stable case may be expressed through the substitution of a quantity $k_{0 s}$ for $k_{0}$ in Prandtl's expressions for mixing length and shear, $k_{0 s}$ being defined through the relation

$$
k_{0 s}=\frac{k_{0}}{u},
$$

$u$ having the same meaning as before, namely, 


$$
u=\sqrt{\frac{1}{2}+\frac{1}{2} \sqrt{\mathrm{I}+\frac{4 \kappa^{2}}{C^{2}}}}=\sqrt{\frac{1}{2}+\frac{1}{2} \sqrt{\mathrm{I}+\frac{4^{\kappa^{2}\left(z+z_{0}\right)^{2}}}{\left(\frac{\mathrm{I}}{k_{0}} \sqrt{\frac{\tau}{\rho}}\right)^{2}}}}
$$

We shall now attempt to extend our survey of the influence of stability to the entire layer of frictional influence. To this end we assume that the correlation coefficient $k$ for the free atmosphere is similarily reduced whenever a stable lapse rate prevails in the atmosphere. Thus

$$
k_{s}=\frac{k}{u_{\infty}} \text { and } K_{s} \equiv \frac{f \sqrt{2}}{3 k_{s}^{2}}=K u_{\infty}^{2}
$$

where $u_{\infty}$ is defined by

$$
u_{\infty}=\sqrt{\frac{1}{2}+\frac{1}{2} \sqrt{\mathrm{I}+\frac{18 \kappa^{2} k^{4}}{f^{2}}}}
$$

For extremely high values of the stability this expression approaches the limit

$$
u_{\infty} \rightarrow \sqrt{\frac{3}{\sqrt{2}}} k \sqrt{\frac{\kappa}{f}} .
$$

Thus, for high values of $\kappa^{2}$ one obtains

$$
k_{s}=\frac{k}{u_{\infty}} \rightarrow \frac{\sqrt[4]{2}}{\sqrt{3}} \sqrt{\frac{f}{\kappa}}=0.687 \sqrt{\frac{f}{\kappa}} .
$$

The formal solution of the equations of motion given in A remains the same as before with the exception that $k_{s}$ everywhere takes the place of $k$. This solution, valid for the free atmosphere, must be patched together with the solution for the surface layer presented in the preceding section. Assuming $\kappa^{2}$ to be constant throughout, one must require continuity in $\tau, l$ and $W$. From these three conditions it follows that the stability factor $(u)$ and the rate of shear are continuous. The condition of continuity in mixing length gives

$$
k_{0 s}\left(H+z_{0}\right)=\frac{k_{s} h}{\sqrt{2}}
$$

or

$$
H+z_{0}=\frac{k}{k_{0} \sqrt{2}} h=0.12 h
$$

The relation between $H+z_{0}$ and $h$ is not affected by stability. From the condition of continuity in wind velocity it follows that the two expressions

$$
W_{H}=U_{g}\left(\cos \varphi_{s}-\frac{\mathrm{I}}{\sqrt{2}} \sin \varphi_{s}\right)
$$


$(\mathbf{1} \circ 3)$

$$
W_{H}=\frac{\mathrm{I}}{k_{0}} \sqrt{\frac{\tau_{0}}{\rho}}\left[\ln \frac{H+z_{0}}{z_{0}}+2(u-\mathrm{I})-\ln \frac{u(u+\mathrm{I})}{2}\right] \quad\left(u=u_{H}=u_{\infty}\right)
$$

must be equal.

Since $\tau$ is continuous at $z=H$ one obtains

$$
\sqrt{\frac{\tau_{0}}{\rho}}=\frac{f h}{3 k_{s}}, \quad \frac{\mathrm{I}}{k_{0}} \sqrt{\frac{\tau_{0}}{\rho}}=\frac{f h}{3 k k_{0}} u_{\infty} .
$$

From (A I67) one obtains, after substitution of $k_{s}$ for $k$,

$$
h=\frac{9 k_{s}^{2}}{2 f} U_{g} \sin \varphi_{s}
$$

or

$$
h=\frac{9 k^{2}}{2 f u_{\infty}^{2}} U_{v} \sin \varphi_{s}
$$

Thus

$$
\frac{\mathrm{I}}{k_{0}} \sqrt{\frac{\overline{\tau_{0}}}{\rho}}=\frac{3 k}{2 k_{0}} U_{g} \frac{\sin \varphi_{s}}{u_{\infty}}
$$

Consequently

(I 20) $\cos \varphi_{s}-\frac{\mathrm{I}}{\sqrt{2}} \sin \varphi_{s}=\frac{3 k}{2 k_{0}} \frac{\sin \varphi_{s}}{u_{\infty}}\left[\ln \frac{H+z_{0}}{z_{0}}+2\left(u_{\infty}-\mathrm{I}\right)-\ln \frac{u_{\infty}\left(u_{\infty}+\mathrm{I}\right)}{2}\right]$.

It is easily seen that

$$
\frac{H+z_{0}}{z_{0}}=\frac{k h}{k_{0} \sqrt{2} \cdot z_{0}}=\frac{9 k^{3}}{2 \sqrt{2} k_{0}} N \frac{\sin \varphi_{s}}{u_{\infty}^{2}} \quad\left(N=\frac{U_{g}}{f z_{0}}\right)
$$

It follows that

$$
\begin{aligned}
\ln N= & \frac{2 k_{0}}{3 k} u_{\infty}\left[\cot \varphi_{s}-\frac{\mathrm{I}}{\sqrt{2}}\right]-\ln \sin \varphi_{s}-\ln \frac{9 k^{3}}{2 \sqrt{2} k_{0}} \\
& +\ln \frac{u_{\infty}{ }^{3}\left(u_{\infty}+\mathrm{I}\right)}{2}-2\left(u_{\infty}-\mathrm{I}\right)
\end{aligned}
$$

or

$$
\begin{aligned}
\log N & =\mathrm{r} .694 u_{\infty}\left[\cot \varphi_{s}-0.707 \mathrm{I}\right]-\log \sin \varphi_{s} \\
& +\log \frac{u_{\infty}{ }^{3}\left(u_{\infty}+\mathrm{I}\right)}{2}-0.8686\left(u_{\infty}-\mathrm{I}\right) .
\end{aligned}
$$

This solution is represented in Fig. 7 for various values of the parameter $u_{\infty}$. The relation between $u_{\infty}$ and $\kappa^{2} / f^{2}$ is represented in Figs. $22 a$ and $b$. 
As the stability $\kappa^{2}$ and therefore $u_{\infty}$ increase, $\varphi_{s}$ approaches a certain limiting value, obtained from

$$
\frac{2 k_{0}}{3 k} u_{\infty}\left(\cot \varphi_{s}-\frac{\mathrm{I}}{\sqrt{2}}\right)-2 u_{\infty}=0
$$

or

$$
\cot \varphi_{s}=\frac{\mathrm{I}}{\sqrt{2}}+\frac{3 k}{k_{0}}=\mathrm{I} .22 \mathrm{I}, \quad \varphi_{s}=39^{\circ} \cdot 3
$$
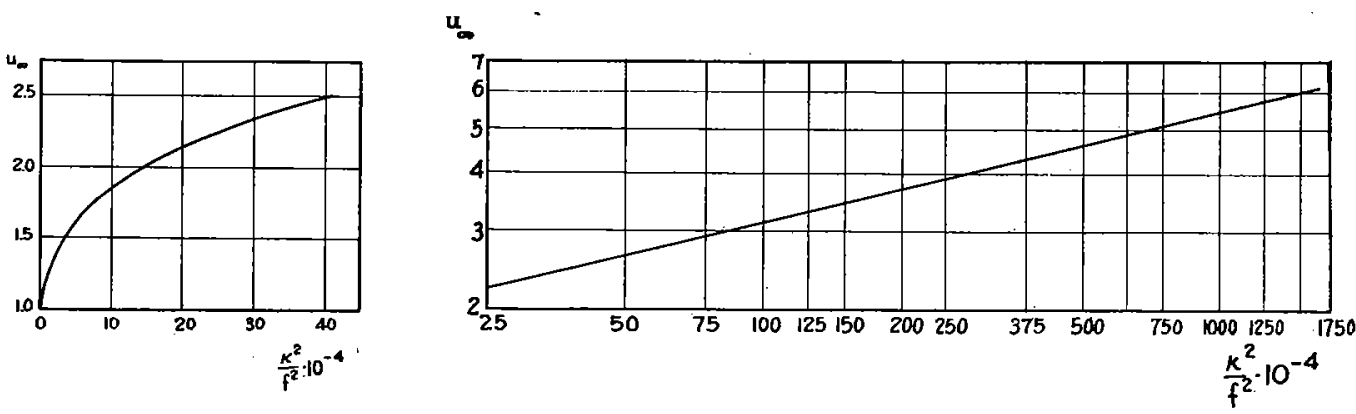

FIGS. 22a and 22b.--Relation between $u_{\infty}$ and $\kappa^{2} / f^{2}$.

Introducing the asymptotic expression for $k_{s}\left(u_{\infty}\right)$ one obtains

$$
\begin{aligned}
h & =\frac{9}{2 f} \frac{\sqrt{2}}{3} \frac{f}{\kappa} U_{s} \sin \varphi_{s} \\
& =\frac{3}{\sqrt{2}} \frac{U_{g}}{\kappa} \sin \varphi_{s}=2.12 \frac{U_{g}}{\kappa} \sin \varphi_{s} .
\end{aligned}
$$

Thus the total height of the layer of frictional influence is given by

$$
H+h=2.37 \frac{U_{g} \sin \varphi_{s}}{\kappa}
$$

The usefulness of the last relation is severely restricted by the fact that in most cases with extremely stable stratification but moderate or strong winds there is likely to be a layer next to the ground in which normal lapse rates prevail.

The asymptotic value of the stress at the ground is given by

$$
\sqrt{\frac{\tau_{0}}{\rho}}=\sqrt[4]{\frac{9}{8}} \sqrt{\frac{f}{\kappa}} U_{o} \sin \varphi_{s}=1.03 \sqrt{\frac{f}{\kappa}} U_{g} \sin \varphi_{s}
$$

The asymptotic maximum value of the eddy-viscosity coefficient is given by

$$
\eta=\frac{3 \rho f}{2 \kappa^{2} \sqrt{2}} U_{g}^{2} \sin ^{2} \varphi_{s}=\mathrm{I} .06 \cdot \frac{\rho f}{\kappa^{2}} U_{g}^{2} \sin ^{2} \varphi_{s} .
$$


Thus, for high values of the stability $\kappa^{2}$, the eddy-viscosity becomes inversely proportional to $\kappa^{2}$.

The most striking result of the theory presented above is the existence of a limiting value for $\varphi_{s}$, the angle between the surface wind and the gradient wind. This limiting value is independent of $\beta$, the stability coefficient, for which we have preliminary estimates only and depends solely on $k$ and $k_{0}$. These two constants are fairly well known from determinations of the height (depth) of the homogeneous layer in air and water; it should therefore be permissible to place some confidence in the numerical value derived above.

In Fig. $23 u_{\infty}$ is plotted against the lapse rate $\alpha=-d T / d z$ from the equation

$$
u_{\infty}=\sqrt{\frac{1}{2}+\frac{1}{2} \sqrt{\mathrm{I}+\frac{\mathrm{I} 8 \kappa^{2} k^{4}}{f^{2}}}} \quad \kappa^{2}=\frac{\beta}{g T}\left(\frac{g}{c_{p}}-\alpha\right) .
$$

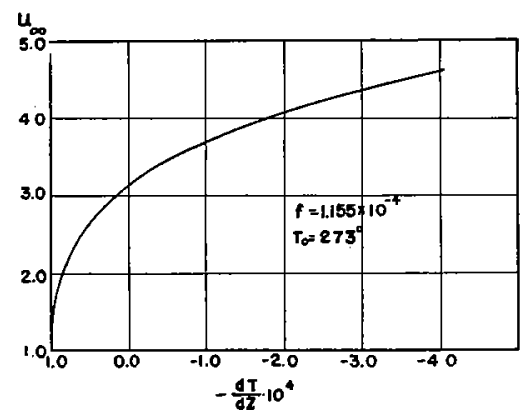

FIG. 23.- $u_{\infty}$ as function of temperature lapse rate.

In computing this curve $\beta$ was assumed to have the numerical value 40 obtained earlier from Heywood's data. The value I.I $55 \cdot 10^{-4}$ was assigned to $f$, corresponding to the latitude of Berlin. Fig. 24 is obtained from Fig. 7 and represents $\varphi_{s}$ as a function of $u_{\infty}$ for different values of $N$. If we set $N=10^{7}$ corresponding to moderate wind velocities in middle latitudes at stations characterized by small roughness parameters $\left(z_{0} \sim \mathrm{I} \mathrm{cm}\right.$. $)$, and combine the two figures in a third (Fig. 25), it appears that the angle $\varphi_{s}$ in-

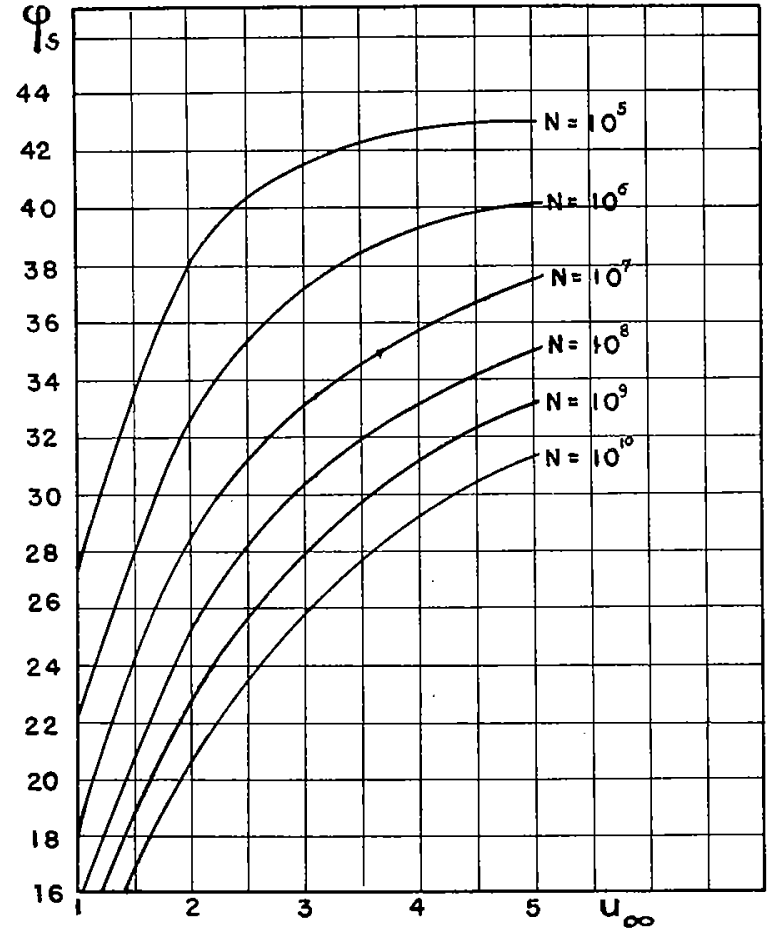

FIG. 24.- Surface wind direction $\left(\varphi_{s}\right)$ as function of stability $\left(u_{\infty}\right)$ and the non-dimensional number $N$. creases at first very rapidly and then more and more slowly with increasing stability. Thus $\varphi_{s}$ increases from a value of $18^{\circ}$ for an adiabatic atmosphere to $30^{\circ}$ for the comparatively steep lapse rate of $0.75^{\circ} \mathrm{C} / 100 \mathrm{~m}$. A further increase in stability to an inversion of $\mathrm{I}^{\circ} \mathrm{C} / 100 \mathrm{~m}$. changes the value of $\varphi_{s}$ to $34^{\circ}$.

It is important to bear in mind that the lapse rate referred to in the preceding discussion is the one characteristic of the entire layer of frictional influence and not just the lapse rate of the first few meters above the ground. On the other hand, it follows from (I 8 b) that the layer of frictional influence contracts so much in depth with even a 
slight degree of stability that it is enough to consider the value of the lapse rate within the first few hundred meters above the ground. To illustrate this contraction of the layer of frictional influence Fig. 26 was constructed. It represents

$$
\frac{h_{\text {stable }}}{h_{\text {adiab }}}=\frac{1}{u_{\infty}^{2}} \frac{\left(\sin \varphi_{s}\right)_{\text {stable }}}{\left(\sin \varphi_{s}\right)_{\text {adiab }}} .
$$

as a function of lapse rate for $N=10^{7}$.

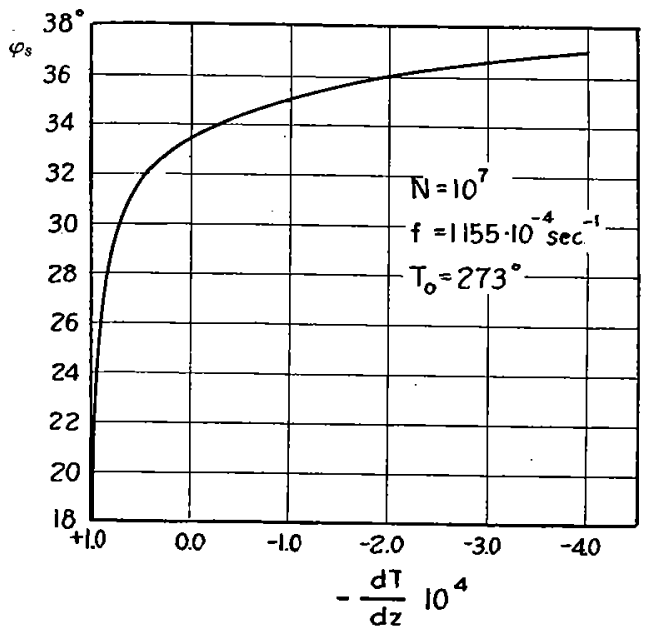

FIG. 25--Surface wind direction $\left(\varphi_{s}\right)$ as a function of stability.

Fig. 27, taken from Hergesell, ${ }^{28}$ gives some information regarding the diurnal variation in the vertical temperature gradient at Lindenberg for different times of the year. It applies to conditions in the first $378 \mathrm{~m}$. above the ground. It is seen that the maximum degree of stability is reached in the early morning and corresponds to an inversion of about $0.1^{\circ} \mathrm{C} / \mathrm{I} 00 \mathrm{~m}$. If the adjustment of the flow to the prevailing state of stability is reasonably ' prompt one should therefore expect to find a total diurnal variation in the angle $\varphi_{s}$ of about $15^{\circ}$, from $\varphi_{s} \sim 18^{\circ}$ in the early afternoon (adiabatic flow) to about $33^{\circ}$ in the early morning (corresponding to a moderate inversion). To verify this prediction we have made use of the observations from Cardington, England, published in Giblett's report on the Structure

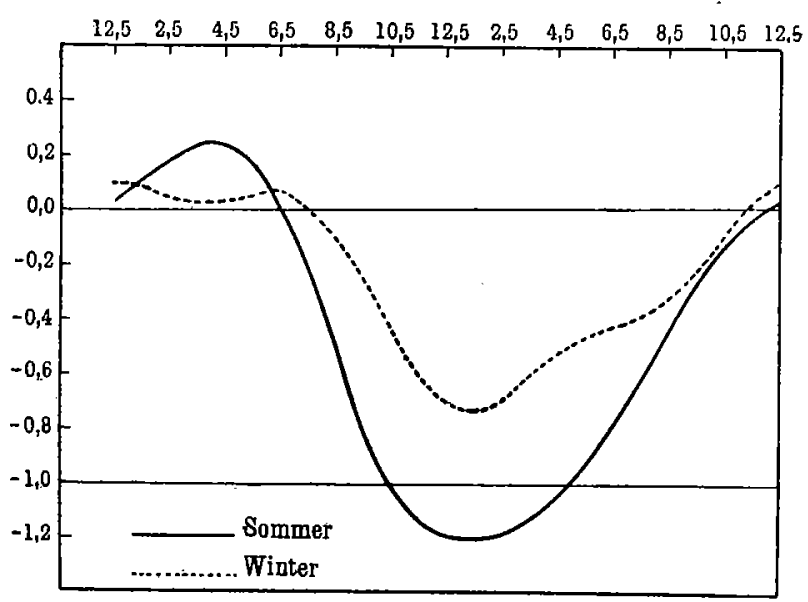

FIG. 27.-Diurnal variation of temperature lapse rate in the first $380 \mathrm{~m}$. above Lindenberg (from Hergesell). IG. 26.- Effect of stable temperature lapse rate
on the depth of the layer of frictional influence.

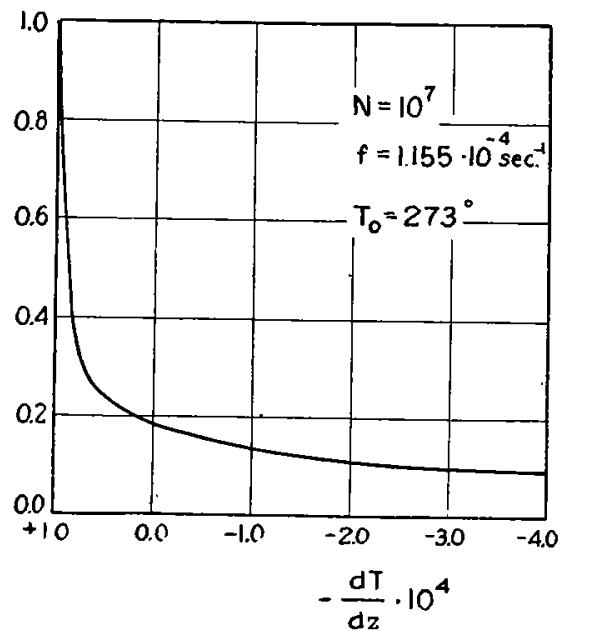

the depth of the layer of frictional influence.

$\frac{h_{\text {stable }}}{h_{\text {adiab }}}$ 
of Wind over Level Country. ${ }^{27}$ Table 22 taken from this report, contains the observed frequencies of various angles between the wind at 150 feet and the direction of the isobars. At I A.M. and 7 A.M. the frequency maximum occurs between $30^{\circ}$ and $34^{\circ}$, at I P.M. between $20^{\circ}$ and $24^{\circ}$.

TABLE 22

Frequency of Backing of Wind at i go Feet From Direction of Isobars

(March I929 to February I930)

(From Cardington report)

\begin{tabular}{|c|c|c|c|c|c|c|c|c|c|c|c|c|c|c|}
\hline G.M.T. & $\begin{array}{l}\text { Geo- } \\
\text { strophic } \\
\text { wind }\end{array}$ & 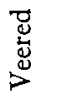 & $0-5$ & $5-9$ & $10-x_{4}$ & I5-19 & $20-24$ & $25-29$ & $30-34$ & $35-39$ & $40-44$ & $45-49$ & $50-54$ & $\begin{array}{c}55 \\
\text { and } \\
\text { over }\end{array}$ \\
\hline & $\begin{array}{c}\text { (mi./hr.) } \\
\text { 10-29 } \\
30-49 \\
50 \text { \& over }\end{array}$ & $\begin{array}{r}\times 5 \\
2 \\
\mathrm{I}\end{array}$ & $\begin{array}{l}7 \\
1\end{array}$ & $\begin{array}{l}8 \\
2 \\
2\end{array}$ & $\begin{array}{r}16 \\
3\end{array}$ & $\begin{array}{r}\text { I2 } \\
6 \\
2\end{array}$ & $\begin{array}{r}10 \\
12 \\
8\end{array}$ & $\begin{array}{l}\text { i } 5 \\
\text { II } \\
7\end{array}$ & $\begin{array}{r}22 \\
6 \\
13\end{array}$ & $\begin{array}{r}22 \\
7 \\
4\end{array}$ & $\begin{array}{r}\mathrm{I} 4 \\
8 \\
\mathrm{I}\end{array}$ & $\begin{array}{r}\text { I2 } \\
2 \\
\text { I }\end{array}$ & $\begin{array}{l}9 \\
3 \\
2\end{array}$ & $\begin{array}{r}28 \\
\mathrm{I}\end{array}$ \\
\hline \multirow[t]{2}{*}{ I GMT } & Total & I8 & 8 & I2 & I9 & 20 & 30 & 33 & 41 & 33 & 22 & I5 & $I_{4}$ & 29 \\
\hline & $\begin{array}{c}10-29 \\
30-49 \\
50 \text { \& over }\end{array}$ & I6 & $\begin{array}{l}5 \\
\text { I }\end{array}$ & $\begin{array}{l}9 \\
2\end{array}$ & $\begin{array}{l}9 \\
4 \\
4\end{array}$ & $\begin{array}{r}15 \\
8 \\
3 \\
\end{array}$ & $\begin{array}{r}22 \\
\text { I I } \\
6\end{array}$ & $\begin{array}{r}20 \\
9 \\
7\end{array}$ & $\begin{array}{r}34 \\
12 \\
2 \\
\end{array}$ & $\begin{array}{r}\text { I } 8 \\
9 \\
\text { I }\end{array}$ & $\begin{array}{r}17 \\
6 \\
3 \\
\end{array}$ & $\begin{array}{r}\text { I } 2 \\
3\end{array}$ & $\begin{array}{r}\mathrm{I} 2 \\
2\end{array}$ & I7 \\
\hline \multirow[t]{2}{*}{$7 \mathrm{GMT}$} & Total & I6 & 6 & II & I7 & 26 & 39 & 36 & 48 & 28 & 26 & 15 & $\mathrm{I}_{4}$ & 17 \\
\hline & $\begin{array}{c}10-29 \\
30-49 \\
50 \text { \& over } \\
\end{array}$ & $\begin{array}{r}22 \\
3 \\
\mathrm{I} \\
\end{array}$ & $\begin{array}{r}\text { I6 } \\
2\end{array}$ & $\begin{array}{r}15 \\
7 \\
\mathrm{I} \\
\end{array}$ & $\begin{array}{r}23 \\
9 \\
3 \\
\end{array}$ & $\begin{array}{r}16 \\
12 \\
2 \\
\end{array}$ & $\begin{array}{l}15 \\
19 \\
10\end{array}$ & $\begin{array}{r}25 \\
4 \\
4 \\
\end{array}$ & $\begin{array}{r}\mathrm{I} 4 \\
8 \\
5 \\
\end{array}$ & $\begin{array}{r}I_{4} \\
6 \\
\mathrm{I} \\
\end{array}$ & $\begin{array}{r}x \text { I } \\
4\end{array}$ & $\begin{array}{r}\mathrm{I} 2 \\
\mathrm{I}\end{array}$ & 2 & $\begin{array}{r}\mathrm{I} 8 \\
\mathrm{I}\end{array}$ \\
\hline \multirow[t]{2}{*}{ I $3 \mathrm{GMT}$} & Total & 26 & 18 & 23 & 35 & 30 & 44 & 33 & 27 & $2 \mathrm{I}$ & 15 & I3 & 2 & I9 \\
\hline & $\begin{array}{r}10-29 \\
30-49 \\
50 \text { \& over } \\
\end{array}$ & $\begin{array}{r}22 \\
2\end{array}$ & $\begin{array}{r}\text { ro } \\
1 \\
2\end{array}$ & $\begin{array}{l}6 \\
3\end{array}$ & $\begin{array}{l}8 \\
6\end{array}$ & $\begin{array}{r}18 \\
7 \\
7 \\
\end{array}$ & $\begin{array}{r}15 \\
7 \\
3\end{array}$ & $\begin{array}{r}23 \\
11 \\
5\end{array}$ & $\begin{array}{r}19 \\
9 \\
6\end{array}$ & $\begin{array}{r}\text { I2 } \\
\text { I0 } \\
3\end{array}$ & $\begin{array}{r}19 \\
2\end{array}$ & $\begin{array}{r}\text { I } 2 \\
5\end{array}$ & $\begin{array}{r}I_{3} \\
5\end{array}$ & $\begin{array}{r}2 \mathrm{I} \\
6\end{array}$ \\
\hline I $8 \mathrm{GM}^{\prime} \mathrm{T}$ & Total & 24 & I 3 & 9 & $I_{4}$ & 32 & 25 & 39 & 34 & 25 & $2 \mathrm{I}$ & I7 & I 8 & 27 \\
\hline
\end{tabular}

Sverdrup's data from the Arctic may also be used as a check on our theoretical determination of $\varphi_{s}$ for great stability. ${ }^{29}$ In most cases he finds a shallow layer of normal lapse rates next to the ice and above this a sharp inversion. In our theoretical treatment we have assumed a constant lapse rate. However, in Table 22 of the paper just referred to, Sverdrup has tabulated the mean vertical wind distribution obtained from 30 pilot balloon runs made on occasions when the inversion began below $100 \mathrm{~m}$. The mean temperature distribution was isothermal to about $50 \mathrm{~m}$. above the ice. From $50 \mathrm{~m}$. to $200 \mathrm{~m}$. the temperature increased from about $-27.4^{\circ} \mathrm{C}$ to about $-20.4^{\circ} \mathrm{C}$. The gradient wind direction is reached in about $200 \mathrm{~m}$. and forms an angle of $34^{\circ}$ with the surface wind. Assuming $f=\mathrm{I} .4 \cdot \mathrm{IO}^{-4} \mathrm{sec}^{-1}, z_{0}=4 \mathrm{~cm}, U_{g}=\mathrm{I} \mathrm{I} .2 \mathrm{~m}$.p.s., we find $N=2 \cdot \mathrm{IO}^{6}$. Assuming furthermore an average lapse rate of $d T / d z=3.6 \cdot 10^{-4} \mathrm{~cm}^{-1}$, it follows that $\kappa^{2}=736 \cdot 10^{-4}$ and $\kappa^{2} / f^{2}=375 \cdot \mathrm{IO}^{4}$. Fig. $22 \mathrm{~b}$ shows that $u_{\infty}=4 \cdot 3$. Knowing $N$ and $u_{\infty}$ we may determine $\varphi_{s}$ from Fig. 24 . The result is $35.5^{\circ}$, in fair agreement with Sverdrup's data.

Another check on the theory may be obtained from the diurnal variation of the wind velocity very close to the ground. It has been mentioned before that this quantity is fairly independent of the lapse rate and given by approximately

$$
W_{z}=\frac{\mathrm{I}}{k_{0}} \sqrt{\frac{\tau}{\rho}} \ln \frac{z+z_{0}}{z_{0}} .
$$


Assuming the diurnal variation in the horizontal pressure gradient to be negligible it follows that the diurnal variation in the surface wind is directly proportional to the diurnal variation in the drag $(\tau)$, which in turn depends upon the diurnal variation in the lapse rate within the entire layer of frictional influence.

It follows from (IIg) that

$$
\frac{W_{\min }}{W_{\max }} \sim \sqrt{\frac{\tau_{\min }}{\tau_{\max }}}=\frac{\mathrm{I}}{\left(u_{\infty}\right)_{\text {stable }}} \cdot \frac{\left(\sin \varphi_{s}\right)_{\text {stable }}}{\left(\sin \varphi_{s}\right)_{\text {adiab }}} .
$$

This ratio has been computed and plotted as a function of lapse rate in Fig. 28. It was again assumed that $N=\mathrm{IO}^{7}, f=\mathrm{I}$. I $55 \cdot \mathrm{IO}^{-4}$ and $T=273^{\circ}$ absolute. From Hellmann's observations at $\mathrm{Nauen}^{9}$ the diurnal variation of the wind velocity at $2 \mathrm{~m}$. above the ground is well known. Unfortunately there are no data available on the diurnal variation of the vertical temperature gradient over Nauen for the same period. We have, therefore, made use of Hergesell's data for Lindenberg and assumed that they are fairly representative of conditions in Nauen. It appears that in winter the daytime lapse rate never fully reaches the dry adiabatic, whereas during the warm half of the year the afternoon lapse rate is well in excess of the dry adiabatic.

In the previous discussion nothing has been said about the wind distribution in the unstable case. It is fairly certain that a major part of the instability is concentrated near the ground, where the presence of a rigid boundary sets a definite limit to the value of the mixing length. We shall, therefore, assume that the vertical velocity distribution near the

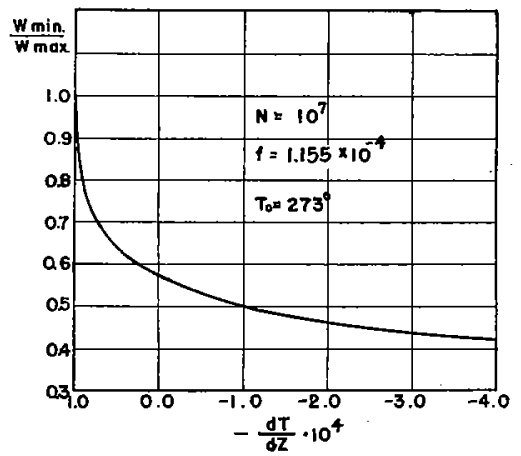

FIG. 28.-Ratio of diurnal minimum wind velocity to diurnal maximum wind velocity for different values of the lapse rate at the time of minimum wind velocity. The diagram applies to velocities measured within the first few meters above the ground. ground is independent of the presence of a slight degree of instability. The correctness of this assumption is supported by an inspection of Heywood's data referred to earlier (p. 5I), which show that the difference in wind velocity between two levels $(95 \mathrm{~m}$. and I $3 \mathrm{~m}$.) remains practically constant throughout the day in spite of the fact that the lapse rate, as indicated by the data from Lindenberg, grows from the adiabatic value around 9:30 A.M. to a superadiabatic maximum at about I P.M. and then decreases to the adiabatic about 5 P.M.

Following the reasoning outlined above, we have determined $W_{\max } / W_{\min }$ for the warm season from Fig. 28 as if the lapse rate had been dry adiabatic at the time of maximum surface wind.

During the winter the lapse rate never fully reaches the adiabatic value. Disregarding possible diurnal variations in $U_{y}$, we have computed $W_{\max } / W_{\min }$ from

$$
\frac{W_{\max }}{W_{\min }}=\frac{u_{\infty \min }}{u_{\infty \max }} \cdot \frac{\left(\sin \varphi_{s}\right)_{\max }}{\left(\sin \varphi_{s}\right)_{\min }}
$$

where $u_{\max }, \varphi_{\text {smin }}$, etc. are obtained from Fig. 23 and Fig. 25 with the aid of Hergesell's maximum and minimum values of the lapse rate. The various values of the lapse rate 
and the computed values of $W_{\max } / W_{\min }$ are given in Table 23 together with Hellmann's observed values (Table I in Hellmann's paper). The agreement is satisfactory.

TABLE 23

\begin{tabular}{|c|c|c|c|c|}
\hline SEAson & $\underset{\operatorname{MAX}}{-d T / d z \cdot 1 \mathrm{O}^{4}}$ & $\underset{\text { MIN }}{-d T / d z \cdot 10^{4}}$ & $W_{\max } / W_{\min }$ & $W_{\max } / W_{\min }$ \\
\hline Winter & 0.65 & -0.02 & I. 19 & $x \cdot 36$ \\
\hline
\end{tabular}

\section{APPLICATIONS TO DRIFT CURRENTS}

\section{General Comments}

There are good reasons to believe that the theory outlined in the preceding sections is applicable also to certain types of oceanic motion. However, due to the scarcity of reliable current measurements it is often difficult or even impossible to verify the theoretical predictions with the aid of direct observations. The difficulties connected with current measurements in the surface layers of a deep ocean are well known. In shallow waters, where conditions are more favourable for measurements, the pure wind current is obtained by eliminating from the observed current tidal and residual permanent current components. In studying these latter drift currents it must be borne in mind that the eddy-viscosity distribution corresponds, not to the wind stirring at the surface but to the incomparably stronger stirring produced by the tides and, tolsome extent, by the permanent currents. Thus drift currents may exhibit quite different characteristics on the continental shelf and in deep water.

Next to the bottom, permanent currents (gradient or convection currents) are retarded by bottom-friction. The resulting velocity distribution should agree with the wind distribution discussed in II, I and 2. However, there is not much evidence of strong gradient and convection currents next to the bottom in deep water. In shallow water, on the other hand, the characteristics of the bottom current will depend less on the turbulence created by the permanent current itself than on the much stronger turbulence created by tidal stirring.

Thus it appears that the applicability of the preceding theory is restricted to pure drift currents in the surface layers of deep ocean regions which are free from strong permanent currents. Even there some modifications may be required to take into account the non-rigid character of the sea surface. In case the ocean is covered with drift ice, other changes are necessary.'

It appears from the preceding discussion that turbulence and currents on the continental shelfs obey other laws and require a different treatment. In a following section some general observations are set forth which seem to provide the starting point for an attack on this particular problem.

The principal results of the drift current theory presented in A may be summarized in the following fashion:

Disregarding at first the effect of the stable sea surface on the surface value of the mixing length, it is found that the latter quantity decreases downward according to the law

$$
l=k(h-2) / \sqrt{2}
$$


where $h$ represents the depth of the drift current layer and $k$ is a non-dimensional constant. $h$ and $w_{0}$, the surface drift velocity, may be computed from the wind stress at the surface with the aid of the formula

$$
\tau_{0}=\rho_{w} \frac{f^{2} h^{2}}{9 k^{2}}=\frac{3}{2} \rho_{w} k^{2} w_{0}^{2} .
$$

Making use of the expression (8) for the wind stress, we find

$$
h=\frac{3 k \gamma}{f} \sqrt{\frac{\bar{\rho}}{\rho_{w}}} W_{0}, \quad \frac{w_{0}}{W_{0}}=\frac{\gamma}{k} \sqrt{\frac{2 \rho}{3 \rho_{w}}} .
$$

The angle $\varphi_{0}$ between the surface drift and the wind is given by

$$
\tan \varphi_{0}=\sqrt{2} \quad\left(\varphi_{0}=54^{\circ} 44^{\prime}\right) .
$$

At the time of writing $A$ it seemed best, for want of a better starting point, to choose a value of $k$ that would make $h$ agree with Ekman's "depth of frictional influence." 30 His expression for this depth, which was not substantiated by observation, was derived on the assumption of a constant eddy-viscosity, giving

$$
D=\frac{\sqrt{2} \pi \tau_{0}}{\rho_{w} f w_{0}}
$$

He evaluates this by means of the wind stress formula

$$
\tau_{0}=32 \cdot \mathrm{IO}^{-7} W_{0}{ }^{2} \quad\left(\gamma^{2}=25 \cdot \mathrm{IO}^{-4}, \rho=\mathrm{I} \cdot 3 \cdot \mathrm{IO}^{-3}\right)
$$

and a value for the wind factor assumed from the observations by Mohn and Nansen

$$
w_{0}=0.0127 \frac{W_{0}}{\sqrt{\sin L}}
$$

giving

$$
D=5.6 \cdot \mathrm{IO}^{-4} \frac{W_{0}}{\Omega \rho_{w} \sqrt{\sin L}}=7.5 \frac{W_{0}}{\sqrt{\sin L}}
$$

using $\rho_{w}=\mathrm{I} .025$; or

$$
D=0.044 \frac{w_{0}}{\Omega \rho_{w}}=590 w_{0}
$$

It follows from (132) that

$$
h=\frac{3 k^{2}}{f} \sqrt{\frac{3}{2}} w_{0} .
$$

If $h$ is to agree with $D$ in middle latitudes, it follows from the two last equations that $k$ must have a value of about 0.12 . However, the two quantities $h$ and $D$ are not entirely comparable; the designation of $D$ as the depth to which the drift current penetrates is entirely arbitrary, since in Ekman's case the current velocity decreases at an exponential rate and approaches zero asymptotically. On the other hand, $h$ has a definite physical meaning, being the finite depth of the drift current layer. 
On the basis of various results in the following part of the paper, we now, however, have sufficient evidence that $k=0.065$ for water just as for air, aside from the fact that it is only logical that such a non-dimensional constant should be independent of the medium for geometrically similar systems. This evidence is based firstly on direct measurements of the depth of the homogeneous layer, which is assumed to coincide with the drift current layer $(h)$. Secondly, this value of $k$ leads to results concerning ice drift which agree closely with observation. Thirdly, the resulting wind factors for open water are also in good agreement with observation.

The term wind factor $(r)$ was first introduced by Thorade ${ }^{31}$ and represents the ratio of the surface drift velocity to the surface wind. According to our theory its value is

$$
\frac{w_{0}}{W_{0}}=\frac{\gamma}{k} \sqrt{\frac{2 \rho}{3 \rho_{w}}}
$$

For $k=0.12$ this ratio is about I.2, or less than indicated by direct observations.

The theoretical value of the maximum mixing length is given by

$$
l_{\max }=k h / \sqrt{2}=\frac{3 k^{2}}{f \sqrt{2}} \sqrt{\frac{\rho}{\rho_{w}}} \gamma W_{0} .
$$

Thus $l_{\max }$ becomes very large in the vicinity of the equator. The roughness of the sea and consequently the mixing length next to the surface do increase with the wind velocity but obviously do not depend on the latitude. For this reason the solution in A was modified with the introduction of a boundary layer wherein the mixing length was assumed to increase linearly with depth. This also led to higher values for the wind factor, increasing towards the equator.

If we now accept the well-established value 0.065 for $k$, the wind factor (I $4 \mathrm{I}$ ) becomes 2.23. This value, when increased by the addition of the boundary layer, seems to agree with the latest determinations of the wind factor. A revised treatment of the boundary layer is given in the Appendix.

If the ocean surface is covered with drift ice, the surface mixing length has a small, constant value, determined by the roughness of the ice. Under those circumstances, pronounced shearing motion must occur immediately below the ice, just as it occurs next to the ground in the air. We shall return to this shearing motion in a following section.

\section{The Homogeneous Layer}

The homogeneous layer of the open ocean is very much like the corresponding layer of the atmosphere. It is characterized by constant temperature and salinity, and results from turbulent mixing which has its origin chiefly in the wind. Secondary sources of this turbulence are (a) convection currents due to instability and (b) tidal or other currents moving over other water bodies; in the open ocean these secondary sources are generally, but not always, negligible compared with the primary source, the wind.

It would also seem that there are effects that tend to dissipate a homogeneous layer. Otherwise its thickness in any locality would correspond to the highest wind ever present there, whereas usually a few days after a decrease in wind the thickness of the layer is observed to have decreased again. It is difficult to form a picture which satisfactorily explains this decrease in thickness, since molecular conductivity and diffusion are obviously inadequate. The "secondary source" (b) of turbulence above mentioned must have 
an occasional effect, since the base of the homogeneous layer is characterized by a sharp discontinuity in density which provides a Gleitfäche on which will arise some small turbulence. This in turn will increase the density of the lower part of the homogeneous layer at the expense of the water below the Gleitfiäche, once the wind-impelled turbulence at this depth becomes negligible.

Another way in which the homogeneous layer may be decreased is by addition of heat at the surface. This heat is distributed throughout the turbulent layer, which after a decrease in wind is shallower than the homogeneous layer, and thus a new thermocline is formed at the base of the turbulent layer. Instances of this are often found, where the remains of the old homogeneous layer lie beneath the new one.

In the steady state the homogeneous, the turbulent, and the drift current layers must coincide. If the turbulence is produced entirely by wind, then the turbulent and drift current layers always coincide. But the homogeneous layer is often considerably deeper or slightly shallower than the others. However, what we measure by means of temperature and salinity observations is the depth of the homogeneous layer, so we have to assume this identical with the depth $h$ of the drift current layer.

By means of previously existing observations we attempt below to verify theoretical relation (133a) between $h, W_{0}$ and $L$ and to determine the constant $k$.

In this discussion we neglect the boundary layer which is treated in the Appendix, and use merely the simple expression (I33a) for the depth of the homogeneous layer. The boundary layer constitutes only about 10\% of the total depth of the layer of frictional influence (see Table 33), hence it is permissible to neglect it in this connection. However, Fig. 29 included here shows the values of $h+H$ from the complete solution (205) in the Appendix.

\section{Analysis of Material}

In order that a deep-sea expedition may be used in this investigation, its records must contain the following:

a) Temperature readings to the modern degree of accuracy $\left(+. \circ 1^{\circ}\right)$ at closely-spaced depths, preferably every 5 or 10 meters down to 50 or IOo meters, with the accompanying salinities.

b) Frequent wind observations for several days preceding each deep-sea station, preferably a complete meteorological log with two- or four-hourly observations.

Of the published and unpublished sources of material readily accessible, only three were found to meet these requirements. Even these three are not completely satisfactory, as may be seen from the following summary:

a) The last cruise of the Carnegie, 1928-1929. Temperature and salinities at depths $0,5,25,50,75$, I00 meters, at some stations more frequent. Meteorological

- observations at Greenwich noon daily and at time of deep-sea stations, which occurred on alternate days. (The meteorological log was destroyed when the ship was lost by fire.) These data, as yet unpublished, were kindly supplied for this investigation by Dr. J. A. Fleming, Acting Director of the Department of Terrestrial Magnetism of the Carnegie Institution of Washington.

b) Cruises of the Discovery and William Scoresby, I926-1929. Temperatures and salinities usually at depths $0,5,10,20,30,40,50,75$, I00 meters. Wind observations at time of stations, usually one or two a day. ${ }^{32}$ 
c) Atlantis cruises I-24, I93I-I934. Temperatures and salinities at depths 0, 50, IOO meters, at some stations more frequent. Meteorological $\log .{ }^{33}$

In order to find the thickness of the homogeneous layer corresponding to any wind speed, it is necessary to have as nearly a steady state as possible. Accordingly only stations have been used for which the wind speed was apparently constant for two days, and

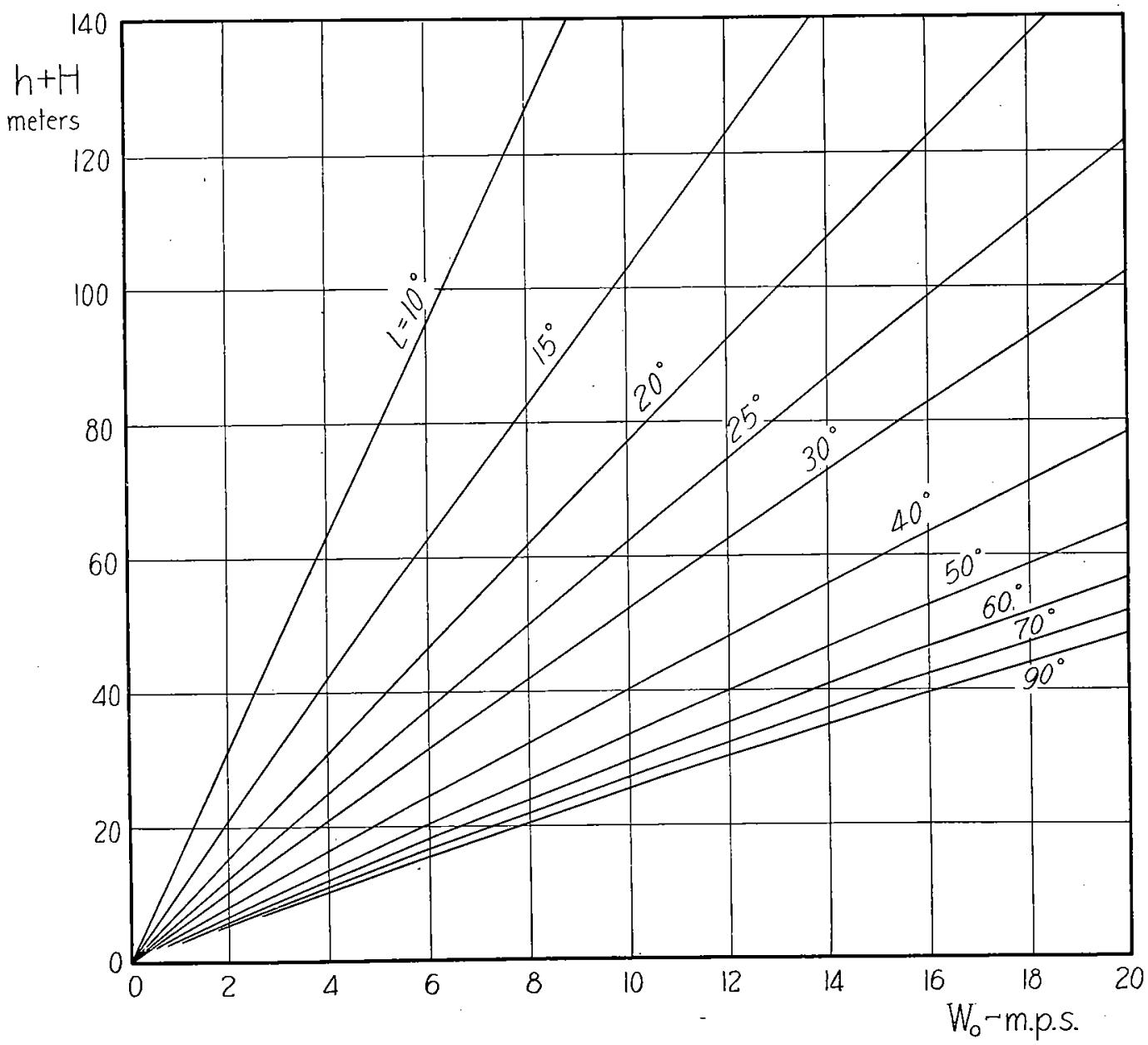

FIG. 29. - Relation between the total depth of the homogeneous layer and the surface wind velocity for different latitudes.

where it had not materially exceeded this steady value for three or four days preceding the station. This seems sufficient time for the homogeneous layer to become established to approximately its normal thickness even in a moderately stable ocean. Stations where instability occurs have not been used.

In each case a smooth temperature-depth curve was drawn, and the depth of the isothermal layer is taken to be the value of $h$. In doubtful cases the isohaline layer has been used as an aid in determining $h$. The isothermal layer is however more reliable, since it is often found that this is shallower than the isohaline, and it is obvious that the 
layer we are interested in must be homogeneous in regard to all its properties. In some cases two or more successive stations occur in the same steady wind; when this happens, only one, usually the last, is included in the analysis below. Furthermore any station (with two exceptions) where the observations are at greater intervals than 25 meters within the homogeneous layer is cast out; and so is any station where the depth of the bottom is less than 1000 meters. Finally, no stations at lower latitudes than $15^{\circ}$ have been used, since theory gives an infinite thickness of the drift-current layer at the equator, which obviously cannot agree with observations.

The limitations indicated above leave only 39 stations that appear suitable for this study. The values found for $h$ at these stations are shown in Table 24 .

It should be noted that we use the expression "homogeneous layer" to mean only the really homogeneous shallow surface layer, and not the whole layer above the principal thermocline, as is done by some oceanographers, which may be several hundred meters deep. Accordingly the layer used in determining the values of $h$ has as a rule no temperature gradients greater than $.0 \mathrm{I}^{\circ} \mathrm{C}$ per meter.

\section{Results of the Analysis}

Lumping the constants together into

we have from (133a) that

$$
C=\frac{3}{2} \frac{\gamma k}{\Omega} \sqrt{\frac{\bar{\rho}^{-}}{\rho_{w}}} \quad(\Omega=\text { angular velocity of the earth }),
$$

(I44) $\quad C=\frac{h \sin L}{W_{0}}$.

( $L=$ latitude).

$C$ was computed for each station by use of Table $25,^{34}$ and the values listed in Table 24 . The average value for $C$ is $2.38 \mathrm{sec}$. The "probable error" of an arithmetic mean is $.6745 \sqrt{\Sigma \Delta^{2}} / N$, where $N=39$ is the number of observations and $\Delta=C-2.38$ is the deviation from the mean..$^{35}$ This comes out to be 0.08 . This value of $C$ determines $k$ to be 0.065 .

If $h$ is actually represented by (I33a) it must be that $C$ is no function of $W_{0}$ or of $L$. To test this, average values of $C$ for each force Beaufort are plotted in Fig. 30 , where the small numbers tell how many observations each point is based on. The curve is irregular, showing no definite trend either upward or downward, so we may conclude that $C$ is independent of $W_{0}$, and hence that $h$ is directly proportional to $W_{0}$. Though Fig. 30 seems to show a very irregular curve, it is well to note that for the three points for which there are more than three observations, the range in $W_{0}$ is about $300 \%$, while the range in $C$ is only about Io\%.

In Fig. 3 I the value of $C$ for each station is plotted against latitude, and a curve

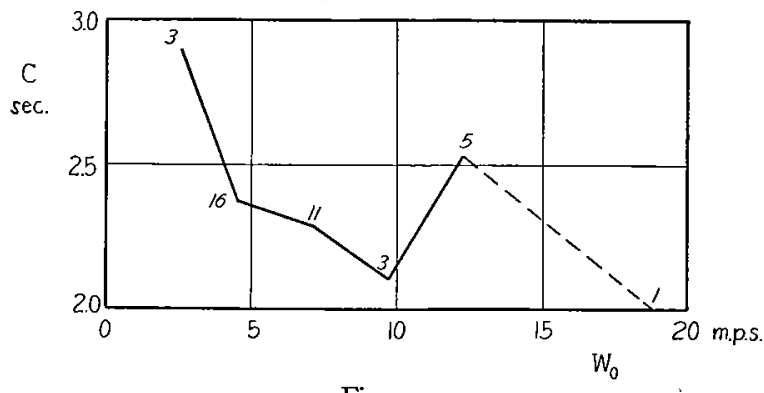

Fig. 30. drawn for the averages of each $10^{\circ}$ interval. The curve shows a slight trend upward to the right, indicating that $n$ is somewhat less than unity in the relation $h \propto \mathrm{I} / \sin ^{n} L$. In order to investigate the value of $n$ more fully, Fig. 32 has been prepared. Here merely $h / W_{0}$, which we know from the preceding paragraph to be the value of $h$ reduced to a standard 


\section{TABLE 24}

\begin{tabular}{|c|c|c|c|c|c|c|c|c|}
\hline Station & Month & $L$ & $\lambda$ & $\begin{array}{l}\text { WIND } \\
\text { B. } \\
\text { Carnegie }\end{array}$ & $\begin{array}{c}h \\
m .\end{array}$ & $\begin{array}{l}\frac{h}{W_{0}} \\
s e c .\end{array}$ & $\begin{array}{c}C \\
\text { sec. }\end{array}$ & $\Delta$ \\
\hline 2 & 5 & $39^{\circ} \mathrm{N}$ & $46^{\circ} \mathrm{W}$ & 6 & 37 & 3.0 & I.9 & $-.4^{8}$ \\
\hline 4 & 5 & $45^{\circ} \mathrm{N}$ & $33^{\circ} \mathrm{W}$ & 4. & 22 & $3 \cdot I$ & 2.2 & -.18 \\
\hline 48 & I I & $19^{\circ} \mathrm{S}$ & I I $4^{\circ} \mathrm{W}$ & 5 & 55 & 5.6 & I.9 & -.48 \\
\hline 59 & 12 & $40^{\circ} \mathrm{S}$ & $10 I^{\circ} \mathrm{W}$ & 3 & 15 & 3.0 & I. 9 & $-.4^{8}$ \\
\hline 61 & 12 & $38^{\circ} \mathrm{S}$ & $94^{\circ} \mathrm{W}$ & 3 & I3 & 2.8 & I. 7 & -.68 \\
\hline 62 & 12 & $35^{\circ} \mathrm{S}$ & $9^{\circ} \mathrm{W}$ & 4 & I8 & 2.5 & $\mathrm{x} .4$ & -.98 \\
\hline 87 & 3 & $\times 8^{\circ} \mathrm{S}$ & $146^{\circ} \mathrm{W}$ & 3 & 35 & 7.6 & 2.4 & .02 \\
\hline $9 \mathbf{I}$ & 3 & $16^{\circ} \mathrm{S}$ & $160^{\circ} \mathrm{W}$ & 4 & 44 & 6.1 & 1.7 & -.68 \\
\hline 102 & 5 & $16^{\circ} \mathrm{N}$ & $172^{\circ} \mathrm{E}$ & 5 & 85 & 8.7 & 2.4 & .02 \\
\hline 105 & 5 & $19^{\circ} \mathrm{N}$ & I $56^{\circ} \mathrm{E}$ & 4 & 62 & 8.6 & 2.8 & .42 \\
\hline IIO & 5 & $26^{\circ} \mathrm{N}$ & $\times 44^{\circ} \mathrm{E}$ & 2 & Io & $3 \cdot 9$ & I. 7 & -.68 \\
\hline xI 5 & 6 & $38^{\circ} \mathrm{N}$ & $\mathrm{I} 45^{\circ} \mathrm{E}$ & 3 & I6 & 3.5 & 2.2 & -.18 \\
\hline 122 & 7 & $46^{\circ} \mathrm{N}$ & ${ }^{\mathrm{I}} 74^{\circ} \mathrm{E}$ & 4 & I7 & 2.4 & 1.7 & -.68 \\
\hline 126 & 7 & $3^{\circ} \mathrm{N}$ & $\mathrm{T}_{43^{\circ} \mathrm{W}} \mathrm{W}$ & 6 & 27 & 2.2 & 1.6 & -.78 \\
\hline I 29 & 7 & $39^{\circ} \mathrm{N}$ & $126^{\circ} \mathrm{W}$ & 6 & 55 & $4 \cdot 4$ & 2.8 & .42 \\
\hline 132 & 9 & $3^{\circ} \mathrm{N}$ & $\mathrm{I} 29^{\circ} \mathrm{W}$ & 3 & 25 & $5 \cdot 4$ & 2.9 & .52 \\
\hline I38 & 9 & $23^{\circ} \mathrm{N}$ & $15 \mathrm{I}^{\circ} \mathrm{W}$ & 4 & 40 & 5.6 & 2.2 & -.18 \\
\hline $\mathrm{I}_{4} \mathrm{I}$ & 10 & $29^{\circ} \mathrm{N}$ & r6 $6 \mathrm{r}^{\circ} \mathrm{W}$ & 5 & $4 \mathrm{I}$ & 4.2 & 2.0 & -.38 \\
\hline I 49 & 10 & $21^{\circ} \mathrm{N}$ & $\mathrm{I} 39^{\circ} \mathrm{W}$ & 4 & 45 & 6.2 & 2.3 & -.08 \\
\hline \multicolumn{9}{|c|}{ Discovery } \\
\hline 85 & 6 & $33^{\circ} \mathrm{S}$ & $5^{\circ} \mathrm{E}$ & 6 & $7 x$ & $5 \cdot 7$ & 3.2 & .82 \\
\hline 98 & 9 & $33^{\circ} \mathrm{S}$ & $I 6^{\circ} \mathrm{E}$ & 4 & 47 & 6.5 & 3.6 & I. 22 \\
\hline 198 & 4 & $63^{\circ} \mathrm{S}$ & $58^{\circ} \mathrm{W}$ & 4 & 22 & $3 . \mathrm{I}$ & 2.8 & .42 \\
\hline \multicolumn{9}{|c|}{ William Scoresby } \\
\hline 36 & I2 & $55^{\circ} \mathrm{S}$ & $35^{\circ} \mathrm{W}$ & 6 & 47 & 3.8 & 3.1 & .72 \\
\hline 317 & I2 & $53^{\circ} \mathrm{S}$ & $50^{\circ} \mathrm{W}$ & 3 & 25 & 5.4 & $4 \cdot 3$ & I. 92 \\
\hline 344 & 1 & $53^{\circ} \mathrm{S}$ & $37^{\circ} \mathrm{W}$ & 3 & 12 & 2.6 & $2 . I$ & -.28 \\
\hline 379 & 2 & $60^{\circ} \mathrm{S}$ & $47^{\circ} \mathrm{W}$ & 3 & 15 & 3.2 & 2.8 & .42 \\
\hline \multicolumn{9}{|c|}{ Atlantis } \\
\hline 1077 & IO & $39^{\circ} \mathrm{N}$ & $73^{\circ} \mathrm{W}$ & 4 & 32 & $4 \cdot 4$ & 2.8 & .42 \\
\hline II 37 & 2 & $36^{\circ} \mathrm{N}$ & $73^{\circ} \mathrm{W}$ & 3 & 25 & 5.4 & 3.2 & .82 \\
\hline I 357 & 8 & $32^{\circ} \mathrm{N}$ & $64^{\circ} \mathrm{W}$ & 2 & 17 & 6.6 & 3.5 & I.I2 \\
\hline I 365 & 8 & $34^{\circ} \mathrm{N}$ & $69^{\circ} \mathrm{W}$ & 4 & 20 & 2.8 & 1.6 & -.78 \\
\hline 1370 & 9 & $36^{\circ} \mathrm{N}$ & $73^{\circ} \mathrm{W}$ & 3 & 15 & 3.2 & 1.9 & $-.4^{8}$ \\
\hline$I_{462}$ & 2 & $35^{\circ} \mathrm{N}$ & $70^{\circ} \mathrm{W}$ & 8 & 65 & $3 \cdot 4$ & 2.0 & -.38 \\
\hline 1470 & 2 & $29^{\circ} \mathrm{W}$ & $72^{\circ} \mathrm{W}$ & 3 & $3 \mathrm{I}$ & 6.7 & 3.2 & .82 \\
\hline 1474 & 2 & $27^{\circ} \mathrm{N}$ & $76^{\circ} \mathrm{W}$ & 3 & 25 & $5 \cdot 4$ & 2.4 & .02 \\
\hline 1477 & 2 & $27^{\circ} \mathrm{N}$ & $77^{\circ} \mathrm{W}$ & 2 & 20 & 7.8 & 3.5 & I.12 \\
\hline $\begin{array}{r}116 \\
\times 548\end{array}$ & 4 & $17^{\circ} \mathrm{N}$ & $79^{\circ} \mathrm{W}$ & 3 & 20 & $4 \cdot 3$ & 1.3 & - I. .08 \\
\hline 1562 & 4 & $20^{\circ} \mathrm{N}$ & $74^{\circ} \mathrm{W}$ & 3 & I9 & 4. I & $\mathrm{I} .4$ & -.98 \\
\hline 1596 & 5 & $19^{\circ} \mathrm{N}$ & $84^{\circ} \mathrm{W}$ & 3 & 28 & 6.1 & 2.0 & -.38 \\
\hline 1598 & 5 & $19^{\circ} \mathrm{N}$ & $85^{\circ} \mathrm{W}$ & 3 & 33 & 7.1 & 2.3 & -.08 \\
\hline
\end{tabular}

TABLE 25

$\begin{array}{ccc}\text { BEAUFORT } & \text { knots } & \text { m.p.s. } \\ 0 & 0 & 0.0 \\ \text { I } & 2 & 1.0 \\ 2 & 5 & 2.6 \\ 3 & 9 & 4.6 \\ 4 & 14 & 7.2 \\ 5 & 19 & 9.8 \\ 6 & 24 & 12.4 \\ 7 & 30 & 15.4 \\ 8 & 37 & 19.0 \\ 9 & 44 & 22.7 \\ 10 & 52 & 26.8 \\ \text { II } & 60 & 30.9\end{array}$


wind speed of $\mathrm{I}$ m.p.s., is plotted against $L$, and the average curve drawn as before. For comparison the curves for the inverse of the sine function and for the inverse of the square root of the sine function are also drawn. For the latter curve the constant 3.2 is used, whereas Ekman ${ }^{30}$ uses 7.5. It is seen that the former fits the average curve more closely, so that $n$ is more nearly equal to $I$ than to $\frac{1}{2}$. In fact the fit is practically perfect except (a) at $20^{\circ}$, which is in such low latitudes that the expression can no longer be expected to hold exactly, and (b) at $60^{\circ}$, for which point there are only $2 \frac{1}{2}$ observations. Of course we could determine $n$ by the method of least squares. But this would not take in to account that there may be a deviation at low latitudes; and, if we use only the 28 stations at latitudes above $25^{\circ}$ for instance, there would be too few to lend accuracy to the determination. In conclusion then it may be said that $n=\mathrm{I}$ within the observational error, except that already at $20^{\circ}$ latitude there appears to be a deviation to lower values of $h$ than are given by a formula such as (I33a).

In order to see the dependence of $C$ on season, average values for each month are plotted in Fig. 33. (Six months were added to each observation in the southern hemisphere.) This shows a sharp drop from about 3 in mid-winter to about 2 in spring, then a gradual rise until midwinter is again reached. The high values in winter are of course due to the small amount of stability accompanying the almost isothermal vertical state of the ocean.

As nearly as can be determined from the observations, then, (I33a) is a true representation of the thickness of the homogeneous layer in a reasonably steady state for latitudes greater than $20^{\circ}$, except that perhaps a small allowance must be made for stability.

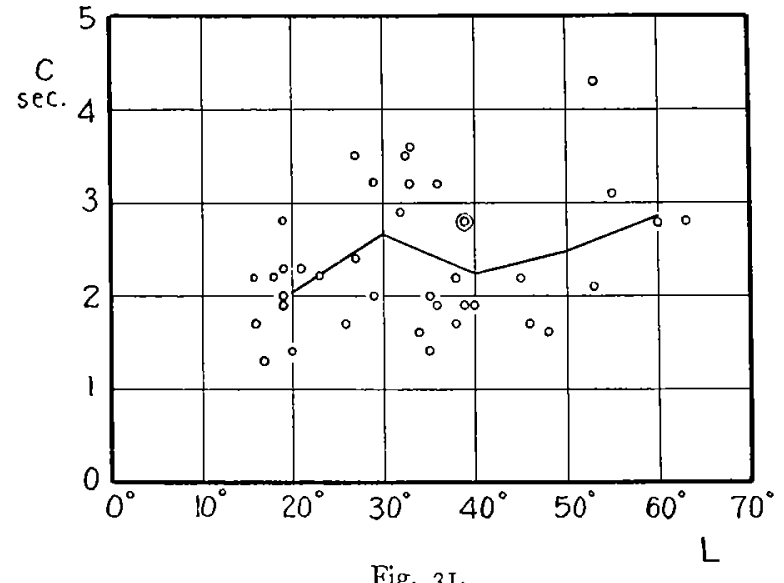

Fig. 3 I.

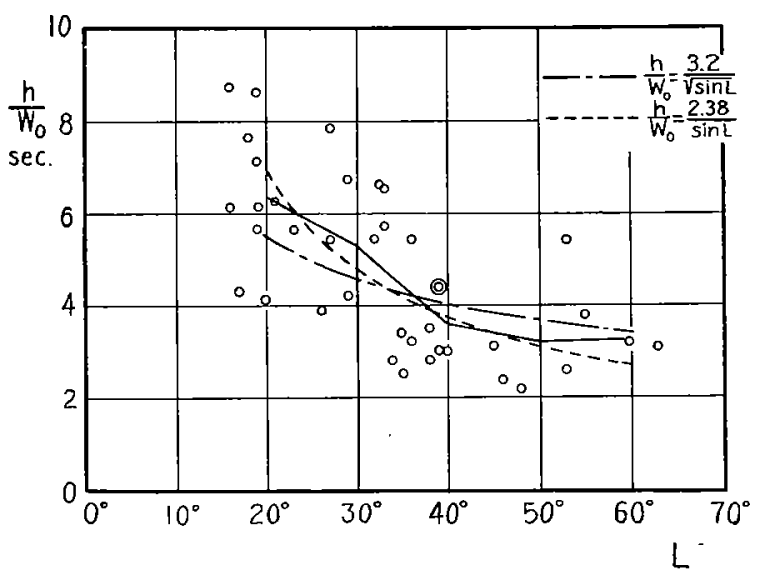

Fig. 32.- Observed ratios between the depth of the homogeneous layer and the surface wind velocity plotted against latitude.

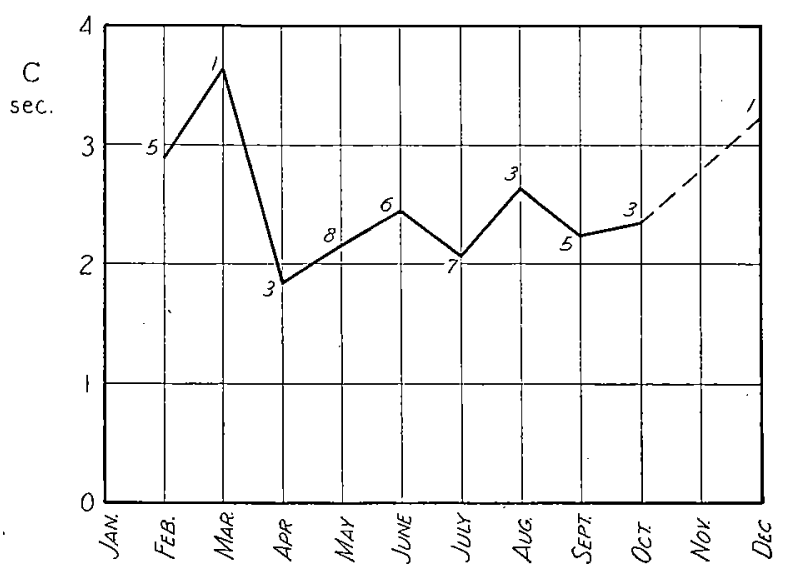

FIG. 33 . 


\section{Scattering of the Observations and Advection}

It is seen from Table 28 and the figures that the individual observations show a good deal of scattering. The maximum deviations for the constant $C$ are - I.I and + I.9. The mean deviation, $\Sigma|\Delta| / N$, has the value .6 or $25 \%$. The causes for this scattering may be classified as follows:

A) Observational inaccuracy

a) Wind is recorded only to the nearest Beaufort unit. An error of one unit, which may occur frequently, represents $50 \%$ at force 3 or $23 \%$ at force 6 .

b) In determining $h$ it is necessary to interpolate between readings of temperature and salinity which are 10-25 meters apart. An error of 5 meters in this interpolation, which is a plausible assumption for the average error, represents $15 \%$, since the average value of $h$ for the 39 observations is 32 meters.

B) We have assumed that the steady state is reached after two or three days. This may not be sufficient time in summer when there is great stability. Furthermore of course the wind is never actually steady for any moderate length of time-there can never be more than a rough approximation to the steady state. We have chosen two or three days as the longest time for which there can be a reasonably steady state.

C) Dependence of $h$ on other independent variables

a) Stability.

b) An important factor we have not yet mentioned is advection.

We have approached this investigation of the homogeneous layer from the viewpoint that it is essentially a problem concerned with a definite vertical column of water. There are many localities where this viewpoint is incomplete; the additional factor that must be taken into account is advection.

We are interested in two forms of oceanic advection. The first is referred to as convergence and divergence. In this case one stratum of water is deepened by convergence from the surroundings, which is compensated for by a divergence to the surroundings in some other stratum. The second form consists of stationary currents. In the latter case the water in one or more strata is continually renewed. The properties of the water in each stratum are unaltered by either of these forms of advection. There is of course a third form, which substitutes a new water body for one of the strata formerly presentthis has a much more important parallel in the atmosphere.

Either of these two forms may affect the depth of the homogeneous layer. A good example of the second form is the case where the shallow edge of a warm current overrides a cold current. Here the homogeneous layer cannot penetrate the boundary between the two currents, and hence is limited to the warm current.

The homogeneous layer is generally identical with the drift current layer. Hence with a uniform wind distribution there is a piling-up or convergence of the homogeneous water on some coastlines and a divergence along others. Or, if the wind distribution is not uniform, divergence occurs in cyclonic regions and convergence in anticyclonic regions. In any case, convergence of the drift current leads to a deeper homogeneous layer than normal, due to piling-up; and divergence leads to a shallower homogeneous layer, since the homogeneous water is carried away faster than it can be maintained down to its normal level. 
Very little is known about the magnitude of this convergence and divergence of the drift current. The upwelling of cold water off the Pacific coast is generally attributed to coastal divergence due to prevailing northerly winds. But it is not known, for instance, whether the semi-permanent, or the migrating cyclones and anticyclones have the greater effect.

\section{Oceanograph Observations}

During the summer of 1934 we have been able to secure some observations on the homogeneous layer by use of an instrument tentatively named "oceanograph," analogous
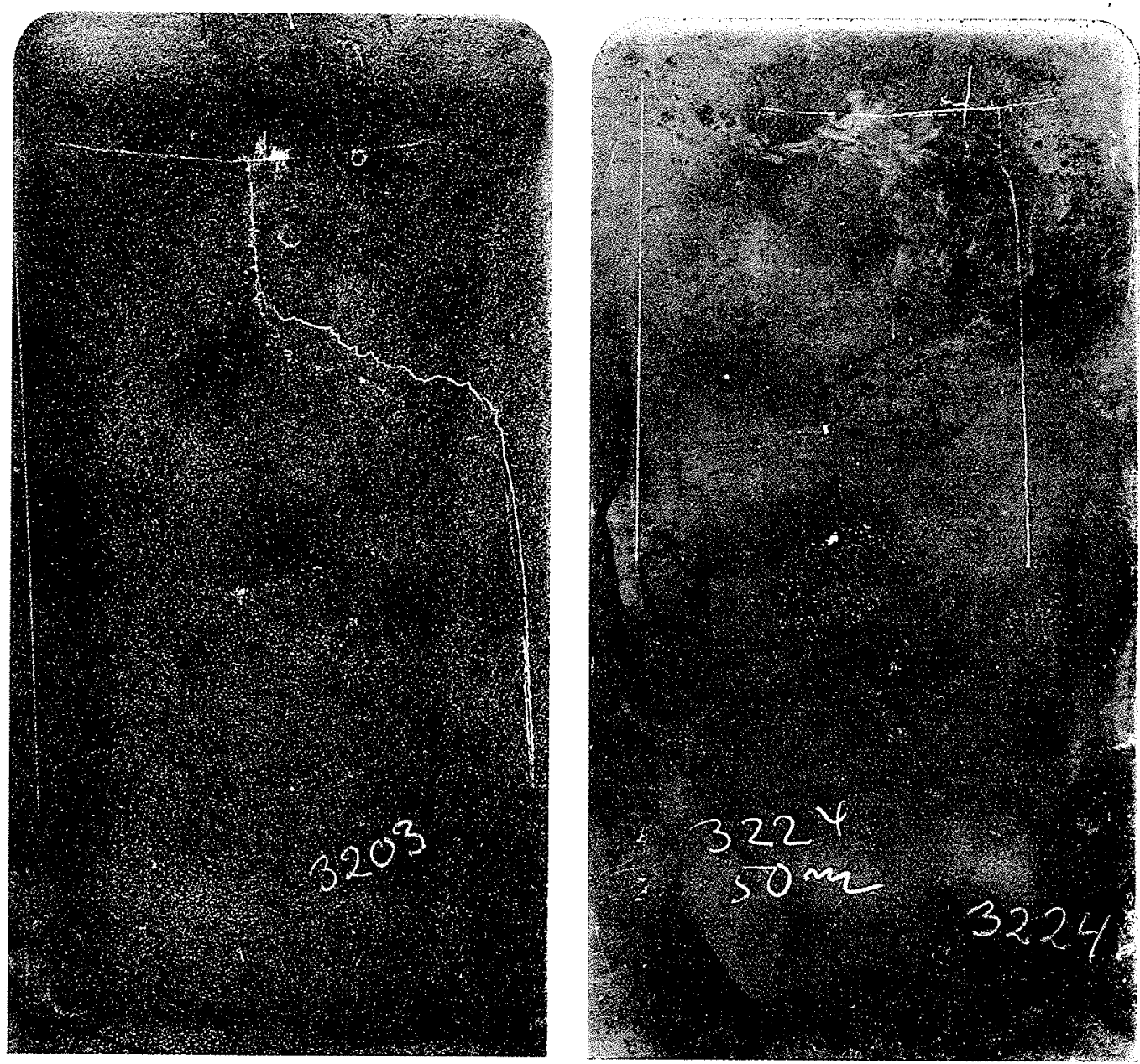

FIG. 34.--Two examples of temperature-depth traces obtained with the oceanograph, reproduced full size.

to the meteorograph used for aerological observations. The water pressure, by means of an aneroid or bellows, moves a plate longitudinally, which bears a smoked brass foil. The temperature is recorded by a bimetal thermometer which records with a stylus across the plate, and is measured off from a straight line drawn by a fixed stylus.* The result is essentially a temperature vs. depth curve.

* The oceanograph is still in the formative stage. It was designed at the authors' suggestion by Dr. K. O. Lange. An improved model is now being constructed and will be tried out during the summer. A complete description of the instrument will be published as soon as it has been developed to a satisfactory degree. 
Two representative foils are reproduced in Fig. 34. Temperature decreases to the right and depth increases downward. The straight line on the left is the temperature reference line. The faint arc at the top is the depth reference line, representing the surface, drawn with a template. One centimeter of the depth scale corresponds to about 7 meters, and gives readings accurate within one meter. One centimeter of the temperature scale corresponds to about $5^{\circ} \mathrm{C}$, and can be read to $\frac{1}{10}$. The bimetal has a very small lag, and the instrument can be used successfully at lowering speeds greater than I m.p.s.

The first of the foils shown has two traces, the upper one being descent and the lower one ascent, the difference being due to hysteresis of the aneroid. Only the descent is used in evaluation, and usually, as in the second foil shown, the stylus is released by a messenger before the instrument is heaved up.

It is obvious that such a continuous record has the advantage of giving the exact depth of temperature discontinuities. The first shows homogeneity down to 15 meters and then a steep thermocline of $13^{\circ} \mathrm{C}$. in the next 15 meters. The wind at this time was 7 m.p.s., the irregularities of the curve being due to motions of the boat and to vibratiosn in the cable supporting the instrument. This is a case where the stability is too great for the homogeneous layer to penetrate to its normal depth corresponding to the wind speed. The second foil shows a much smaller thermocline which is purely the result of windproduced turbulence.
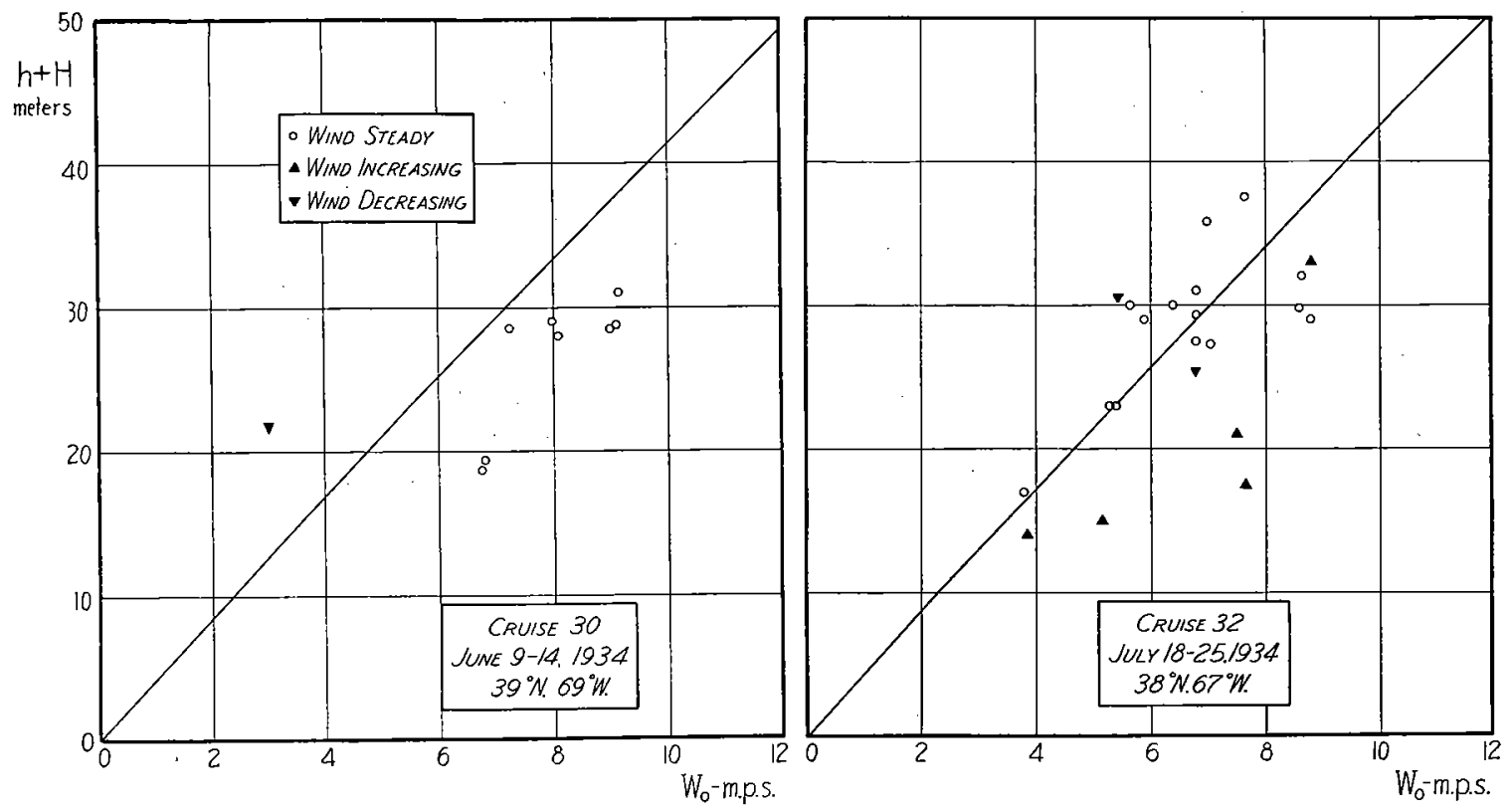

Figs. $35 \mathrm{a}$ and $35 \mathrm{~b}$.-Observed depths of the homogeneous layer plotted against surface wind speed. (Atlantis cruises 30 and 32 ). The lines represent the theoretical relation (205).

In Figs. $35 \mathrm{a}$ and $\mathrm{b}$ are shown the observations made on two short cruises of Atlantis. Most of the points are means of several observations made during a short period. They are plotted against the mean wind speed during the previous 6 hours, observed by means of a four-cup portable (Casella) anemometer, usually held 2 meters directly above the windward rail in the bow (or 5 meters above the sea surface). The scale readings of the 
anemometer have been corrected according to a subsequent calibration. The curve drawn in each figure is the theoretical relation (205) for the mean latitude of the observations.

The homogeneous layers found during the June cruise are all slightly shallower than the theory indicates. This is probably due to unusual stability, resulting at least in part from vernal warming. The values for steady wind from the July cruise, however, lie very

close to the theoretical line. With only one exception the points for increasing wind are below, and those for decreasing wind above, the theoretical line for the steady state.

These few observations then serve as a check on our determination above of the depth of the homogeneous layer from water-bottle observations.

\section{Wind Drift of the Ice}

The wind drift of the ice in Arctic and Antarctic waters has been observed in great detail on several polar expeditions. The data thus collected offer a good opportunity for verification of the theory outlined in this paper. Sverdrup ${ }^{36,37}$ has analyzed his own and Brennecke's ${ }^{38}$ ice drift observations theoretically and introduced new view points which are fundamental to the understanding of the problem. There are however certain discrepancies between Sverdrup's conclusions and our theory which necessitate a reexamination of this question.

In the steady state, the movement of the ice is affected by the following forces:

I. The frictional force $\tau_{a}$ between the air and the ice (directed along the wind).

2. The deflecting force $D$ due to the rotation of the earth (normal to the direction of the ice drift, proportional to the latter and to the mass of the ice).

3. The frictional force between ice and water $\left(\tau_{0}\right)$.

4. An internal frictional force $R$ in the ice, caused by ice meeting ice in a different state of motion. This lack of uniformity in the movement of the ice is mostly the result of horizontal variations in wind direction and wind velocity. Sverdrup assumes $R$ to be proportional to the ice drift and to act in the opposite direction.

The above four forces were introduced by Sverdrup and considered by him in his theoretical discussions. The balance of these four forces is illustrated in Fig. 36a and b.

There are two methods of approach to the problem.

We may disregard the mass of the ice and consider it as a thin film moving with the surface water. We may then neglect the deflecting force acting on the ice. The problem resolves itself into a determination of the characteristics of a drift current generated by the frictional force between ice and water. The latter frictional force may be computed from the wind force since the three forces $\left(\tau_{a}, \tau_{0}\right.$ and $\left.R\right)$ acting on the ice must balance each other.

The second method of attack is based on the assumption that the frictional force between ice and water may be neglected, i.e., the mass of the drift current layer is neglected. The steady motion of the ice is determined from the condition that the remaining forces $\left(\tau_{a}, D\right.$ and $R$ ) balance each other.

Both methods of attack have been followed by Sverdrup, the first in his analysis of Brennecke's data from the Weddell Sea, the second in his analysis of the ice drift on the North-Siberian shelf. The justification for the use of two entirely different methods and assumptions is the following:

The ice in the Weddell Sea is normally fairly thin, about one meter in thickness. There are large lanes between the ice floes and small internal ice resistance. Thus the wind force is readily transmitted to the underlying water. The ice on the North-Siberian shelf 
is thick, varying between two meters in the late summer and three and a half meters in the late winter. The internal ice resistance is great, reducing the drift and making the resistance between ice and water insignificant.

The principal reason why the ice drift has received so much attention in the past lies in the fact that the angle between the drift vector and the wind vector is much less than the value given by Ekman's theory. This discrepancy is successfully explained by Sverdrup as a result of the internal friction in the ice itself. However, since the introduction of a frictional force obviously tends to reduce velocities while on the other hand the observed wind factor in the Weddell Sea is somewhat larger than over the open ocean, Sverdrup's
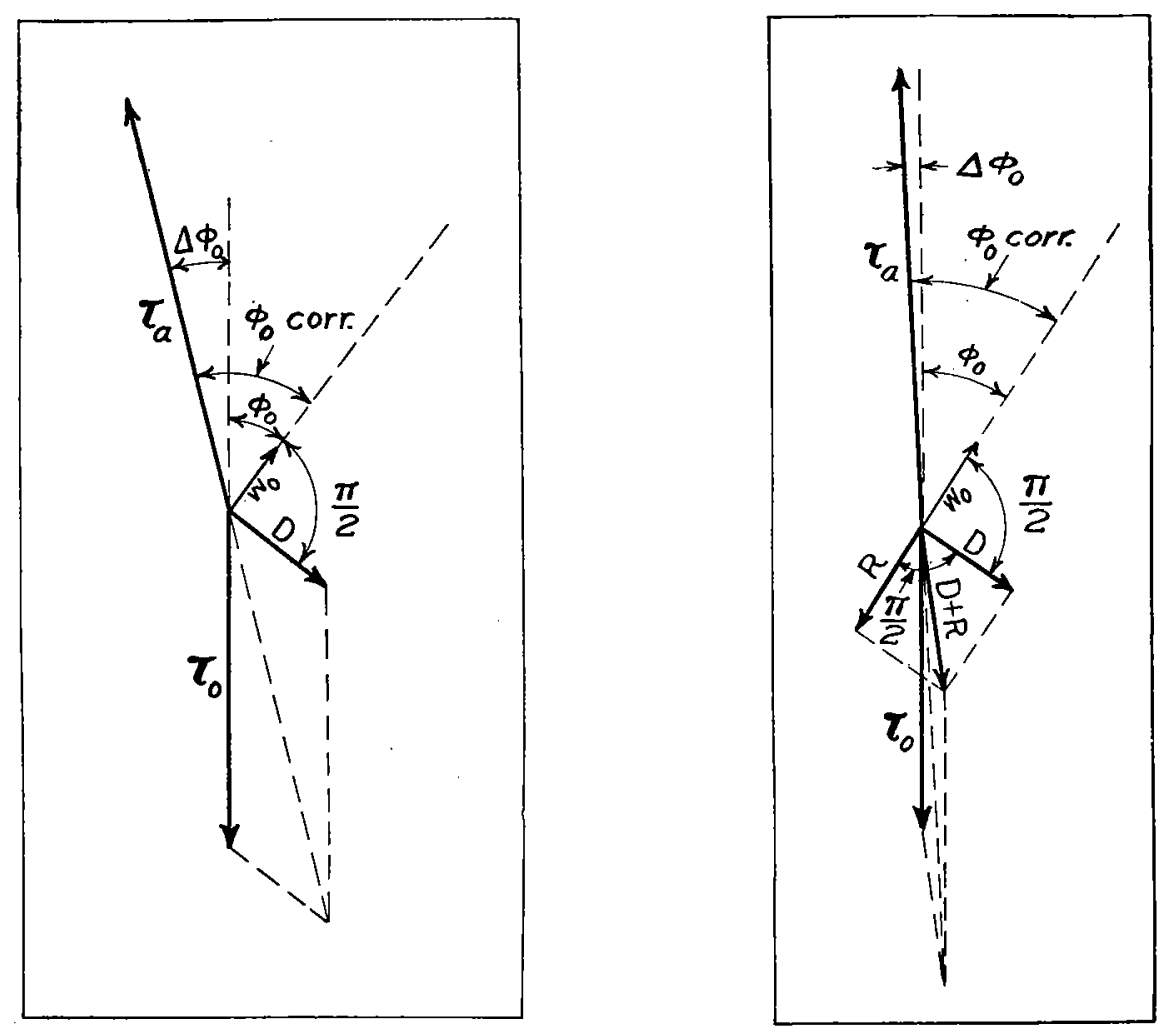

Figs. 36a AND 36b.-Balance between the forces controlling the ice drift. In $32 \mathrm{~b}$ the internal ice resistance $(R)$ has been included to illustrate the effect of this force on the angle $\varphi_{0}$ between ice-drift and wind.

method of analysis leads him to values for the frictional force of the wind which are several times as large as the values used by Ekman and Taylor. According to Sverdrup, the wind force per unit area of ice over the Weddell Sea is proportional to $W_{0}^{3 / 2}$. The wind force per unit mass of ice on the North-Siberian shelf he assumes to be proportional to $W_{0}$. In both cases the proportionality factors are at first left undetermined and then used to fit the theoretical curves to the observations. Sverdrup suggests that the high value of the wind factor on the Weddell Sea is due to the better "grip" of the wind on the ice as compared to the sea. However, Taylor and Ekman have obtained one and the same law for the frictional force of the wind, one over open grass land, the other over the sea. It is not likely that the surface of the ice should produce such pronounced increase in wind 
friction, particularly since the normally fairly stable temperature stratification over the ice tends to reduce the frictional force corresponding to any given wind velocity.

The difficulty is easily avoided if one takes into account the existence of a layer of rapid shearing motion in the water just below the ice. We have stated before that such shear must occur on account of the small value of the mixing length at the under side of the ice. The shear must be pronounced in the Weddell Sea where the ice resistance is small but insignificant in the Arctic where the ice resistance is great.

Our first task is to determine the value of the frictional force of the wind on the ice. Sverdrup obtains, from Brennecke's data,

$$
\tau_{0}=3 \cdot 36 \cdot 10^{-4} W_{0}^{3 / 2} \text {. }
$$

This curve is represented in Fig. 37. The same diagram contains the Ekman-Taylor formula

$$
\frac{\tau_{0}}{\rho}=\gamma^{2} W_{0}^{2}=25 \cdot \mathrm{IO}^{-4} W_{0}^{2}
$$

computed on the assumption that $\rho=\mathrm{I} \cdot 4 \cdot \mathrm{IO}^{-3}$. This high value of $\rho$ was adopted to allow for the low air temperature in the Arctic. The figure shows that Sverdrup's values for $\tau_{0}$ are three to five times larger than those obtained from (8).

In order to determine whether the relation between wind and stress over ice differs from the corresponding well established relation over land, we have computed below the value of the surface stress from three of Sverdrup's pilot balloon observations during the "Maud" expedition. The stress is computed with the aid of (I7) and all the pertinent data are contained in Table 26.

TABLE 26

Determination of Frictional Drag Between Wind and Drift Ice from Sverdrup's Observations A. January 2, $1923, \mathrm{I} 6 \mathrm{~h} 2 \mathrm{Im} \mathrm{LMT}, 73^{\circ} 25^{\prime} \mathrm{N}, 171^{\circ} 37^{\prime} \mathrm{E}$ Gr.

Altitude
7
50
100
150
200
250

DeVIation
From Gradient
Wind DirEction
$33^{\circ}$
$29^{\circ}$
$9^{\circ}$
$3^{\circ}$
$0^{\circ}$

SPEED
m.p.s.
5.0
6.8
18.2
17.6
16.8
I6. 6

Wind Component
NormaL To Isobars
m.p.s.
2.72
3.30
2.85
$0.9^{2}$
0.00
0.00

B. April $27, \mathrm{I} 923$, I $2 \mathrm{~h} 27 \mathrm{~m} \mathrm{LMT}, 74^{\circ} 29^{\prime} \mathrm{N}, \mathrm{I} 67^{\circ} 29^{\prime} \mathrm{E} \mathrm{Gr}$.
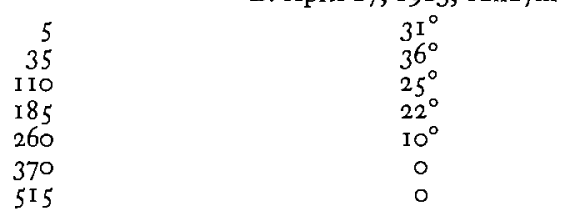

8.8
7.7
11.2
14.0
14.0
13.6
15.4

$$
\begin{aligned}
& 4.53 \\
& 4.53 \\
& 4.74 \\
& 5.25 \\
& 2.44 \\
& 0.00
\end{aligned}
$$

C. January I2, I924, 14 h $33 \mathrm{~m} \mathrm{LMT,} 75^{\circ} \mathrm{O} 8^{\prime} \mathrm{N}, \mathrm{I} 57^{\circ} 30^{\prime} \mathrm{E}$ Gr.

$\begin{array}{rrrr}7 & 48^{\circ} & 3.8 & 2.82 \\ 50 & \mathrm{I}^{\circ} & 6.0 & \mathrm{I} .25 \\ 100 & 6^{\circ} & 12.6 & \mathrm{I} .32 \\ 150 & 4^{\circ} & 12.0 & 0.84 \\ 200 & 3^{\circ} & 10.5 & 0.55 \\ 250 & 3^{\circ} & 9.8 & 0.34 \\ 300 & 2^{\circ} & 9.6 & 0.16 \\ 350 & 1^{\circ} & 9.5 & 0.16 \\ 400 & \mathrm{I}^{\circ} & 9.5 & 0.00\end{array}$

The wind velocities and wind directions in A and C were obtained from Fig. 48 in Sverdrup's discussion of the meteorological results of the "Maud" expedition. ${ }^{29}$

The data in B were taken directly from Sverdrup's original tables. ${ }^{39}$ 
On January 2, I923, the surface wind, measured $7 \mathrm{~m}$. above the ice, was 5 m.p.s. From the computation it follows that $\tau_{0} / \rho=680 \mathrm{~cm} .{ }^{2} / \mathrm{sec}^{2}$ and $\tau_{0} / \rho W_{0}^{2}=\gamma^{2}=27.2 \cdot \mathrm{IO}^{-4}$, in good agreement with Taylor's results. In the second case the surface wind was 8.8 m.p.s. and $\tau_{0} / \rho=2013 \mathrm{~cm} .{ }^{2} / \mathrm{sec}^{2}$. This gives $\gamma^{2}=26.10^{-4}$. In the third case, the surface wind was 3.8 m.p.s., $\tau_{0} / \rho=628$ and $\gamma^{2}=43.5 \cdot 10^{-4}$. The last value for $\gamma^{2}$ is definitely too

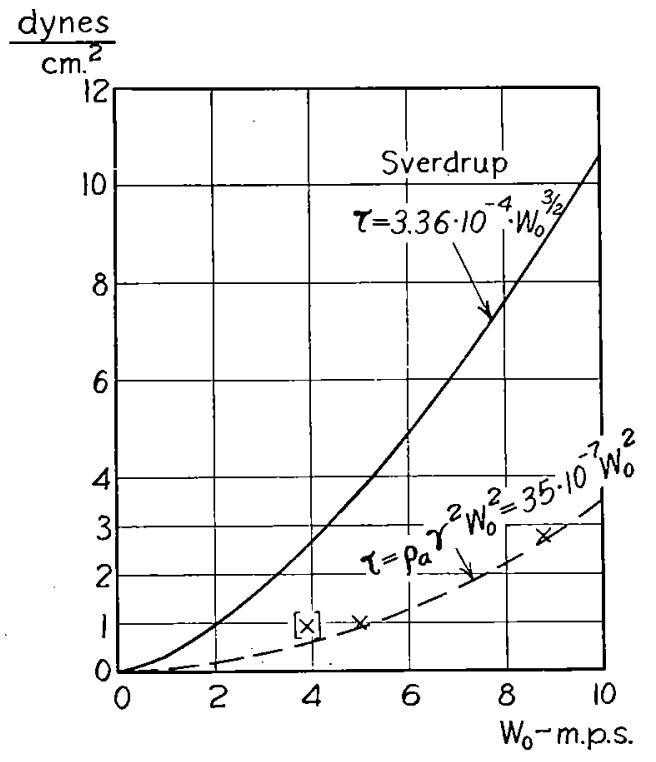

FIG. 37.-Wind force per unit area according to Sverdrup (full line), Ekman-Taylor (broken line). The crosses represent direct determinations from Sverdrup's pilot-balloon data. high. An inspection of the wind distribution on this particular occasion reveals that the gradient wind apparently kept turning right with elevation. This view is supported by the abnormally large value for the angle between the surface wind and the assumed gradent wind.

The three crosses in Fig. 37 indicate the stress values determined above. Of these three direct determinations one is indicated as unreliable for reasons set forth above. We are forced to conclude that the evidence shows the Ekman-Taylor formula to be substantially cor rect and Sverdrup's expression to be several times too large.

Since in the above cases the surface wind was measured fairly close to the ground one should expect $\gamma^{2}$ to be somewhat greater than indicated by Taylor. On the other hand, the vertical temperature gradient next to the ice is nearly always stable, thus tending to reduce the stress corresponding to any given wind velocity. The two effects tend to cancel each other and thus we are justified in using the formula

$$
\frac{\tau_{0}}{\rho}=25 \cdot 10^{-4} W_{0}^{2}
$$

to compute the frictional force over the drift ice.

We shall now attempt to compute the characteristics of the drift current in the Weddell Sea. As a first approximation we assume that the sole effect of the ice is to introduce a layer of shearing motion next to the surface, i.e., we neglect the internal ice resistance and the deflecting force on the ice.

At the depth $H$ below the ice the relation between stress, $\tau_{0}$, and current, $w_{H}$, is given by

$$
\frac{\tau_{0}}{\rho_{w}}=\frac{3}{2} k^{2} w_{H}^{2}=\frac{f^{2} h^{2}}{9^{2}}=\gamma^{2} \frac{\rho}{\rho_{w}} W_{0}^{2} .
$$

The current component in the direction of the wind is

$$
w_{H} \cos \varphi_{H}=\frac{w_{H}}{\sqrt{3}}
$$


The shearing motion in the layer $H$ results in a higher surface drift velocity, $w_{0}$, forming a smaller angle, $\varphi_{0}$, with the wind. We have

$$
\begin{aligned}
& w_{0} \cos \varphi_{0}=w_{H} \cos \varphi_{H}+\frac{\mathrm{I}}{k_{0}} \sqrt{\frac{\tau_{0}}{\rho_{w}}} \ln \frac{H}{z_{0}} \\
& w_{0} \sin \varphi_{0}=w_{H} \sin \varphi_{H} .
\end{aligned}
$$

We know that there must be continuity in the mixing length at the level $H$. Thus

$$
H=\frac{k}{k_{0} \sqrt{2}} h=\frac{3 k^{2} \gamma}{k_{0} \sqrt{2}} \sqrt{\frac{\rho}{\rho_{w}}} \cdot \frac{W_{0}}{f}
$$

$$
\left(n=\frac{3 k^{2} \gamma}{k_{0} \sqrt{2}} \sqrt{\frac{\rho}{\rho_{w}}} \cdot 10^{6}\right)
$$

and

$$
\frac{H}{z_{0}}=n \cdot 10^{-6} \cdot N=425 \cdot 10^{-6} N, \quad\left(N=\frac{W_{0}}{f z_{0}}\right)
$$

where $N$ is a non-dimensional number similar to the one introduced above in the meteorological section. It follows that

(I 49) $\tan \varphi_{0}=\frac{\sqrt{2}}{\mathrm{I}+\frac{3^{k}}{k_{0} \sqrt{2}} \ln \left(n \cdot \mathrm{IO}^{-6} N\right)}, \cot \varphi_{0}=0.586\left[2.835+\log \mathrm{IO}^{-6} N\right]$

and

(150) $\quad r=\frac{w_{0}}{W_{0}}=\frac{w_{H}}{W_{0}} \frac{\sin \varphi_{H}}{\sin \varphi_{0}}=\frac{2 \gamma}{3 k} \sqrt{\frac{\rho}{\rho_{w}}} \frac{\mathrm{I}}{\sin \varphi_{0}}=\frac{\mathrm{I} .8 \cdot 10^{-2}}{\sin \varphi_{0}}$

These two relations between $r, \varphi_{0}$ and $N$ are tabulated below. Knowing the value of $z_{0}$, the roughness of the under side of the ice, we may plot the same quantities against wind velocity. In Brennecke's case $f=\mathrm{I} .36 \cdot \mathrm{IO}^{-4}$. We have chosen for $z_{0}$ a value of $3.68 \mathrm{~cm}$. so that $N=\mathrm{IO}^{6}$ corresponds to a wind velocity of $5 \mathrm{~m}$.p.s. A $z_{0}$-value of $3.68 \mathrm{~cm}$. means api actual roughness of about one meter, which does not seem unreasonable in view of the existence of pressure ridges between ice floes. Fig. 38 contains the theoretical relation between $\varphi_{0}$ and $W_{0}$ and also the results of Brennecke's observations as tabulated by Sverdrup. The agreement is good. Fig. 39 gives the theoretical relation between the wind factor and the wind velocity (upper full line). In the same figure we have entered Sver-

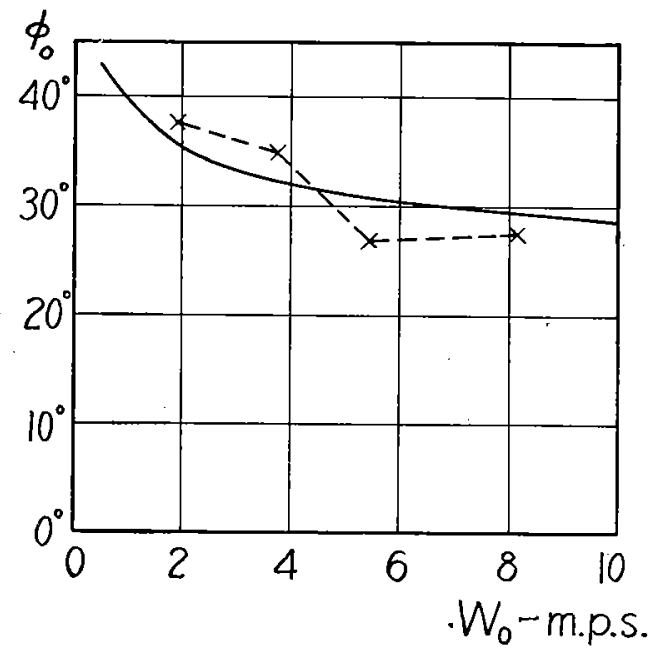

FIG. 38.-The theoretical angle between icedrift and wind plotted against wind velocity (full line). The broken line connects Brennecke's observa- 


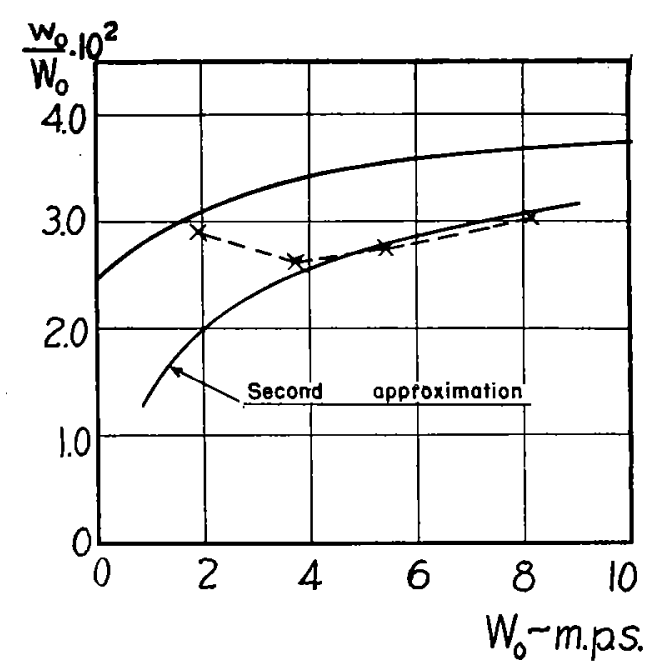

Fig. 39.-Theoretical wind factor plotted against wind velocity (full line). The broken line connects Brennecke's observations.

$$
D=M f w_{0},
$$

where $M$ is the mass of the ice per $\mathrm{cm}^{2}$. This force forms an angle of $\pi / 2+\varphi_{0}$ with the wind direction. The ice resistance is presumably proportional to $w_{0}$ an dacting in the opposite direction. We do not know the magnitude of this force but we shall estimate it in the following way:

The theoretical angle between wind stress and drift vector computed above agrees well with the observations. We shall therefore choose the ice resistance in such a fashion as to leave this angle unchanged, i.e., we assume the resultant of ice resistance and deflecting force to fall in the direction of the frictional force exerted by the water on the ice. It follows that the wind force on the ice $\left(\tau_{a}\right)$ may be computed from $\tau_{0}$ and $D$ by means of the expression

$$
\tau_{a}=\tau_{0}+\frac{M f w_{0}}{\sin \varphi_{0}}
$$

If we indicate by $W_{c}$ the correct wind velocity corresponding to the true wind stress $\left(\tau_{a}\right)$ we find from ( 152 ) and (8)

$$
W_{c}^{2}=W_{0}^{2}\left(I^{3}+\frac{M f w_{0}}{\rho \gamma^{2} W_{0}^{2} \sin \varphi_{0}}\right)
$$

or

$$
W_{c}=W_{0} \sqrt{1+\frac{\overline{M f r}}{\rho \gamma^{2} W_{0} \sin \varphi_{0}}}
$$

we are now in a position to compute the true wind velocity $W_{c}$ corresponding to given values of $r, \varphi_{0}$ and $W_{0}$. Knowing $W_{c}$ we may compute the correct wind factors from

$$
\frac{w_{0}}{W_{c}}=r_{c}=r \cdot \frac{W_{0}}{W_{c}}
$$


The result is presented graphically in Fig. 39 in the curve marked second approximation. The agreement with Brennecke's data is excellent, except for very light winds. The wind factor of light winds will be discussed in connection with the analysis of Sverdrup's data.

Brennecke's ice drift observations may thus be satisfactorily explained from a consideration of the shearing motion immediately below the ice. We can easily estimate the intensity of this shear by comparing the drift velocities at the depth $H$ and at the surface. It follows from ( $\left.\mathrm{I}_{4} 6\right)$ that

$$
\frac{w_{H}}{w_{0}}=\frac{\sin \varphi_{0}}{\sin \varphi_{H}}=\sqrt{\frac{3}{2}} \sin \varphi_{0}
$$

This ratio is entered in Table 27. From a series of current measurements in the surface layer Brennecke finds that the drift two meters below the ice amounts to about $58 \%$ of the surface drift, in very good agreement with our theoretical result.

The depth of the drift current, $H+h$, may be computed from (I47). It follows that

$$
H+h=2.89 W_{0} .
$$

These values have likewise been entered in Table 27. Brennecke found that in most cases the current 25 meters below the ice is negligibly small. This agrees well with the shallowness of the theoretical drift current depths in Table 27.

TABLE 27

$\begin{array}{cccccc}N \cdot I O^{-6} & \varphi_{0}{ }^{\circ} & r \cdot I^{2} & W_{0} \text { (m.p.s.) } & W_{c} \text { (m.p.s.) } & r_{c} \cdot 10^{2} \\ & & & & & \\ 0 . I & 43.0 & 2.65 & 0.5 & 0.97 & 1.37 \\ 0.5 & 34.0 & 3.22 & 2.5 & 3.38 & 2.39 \\ 1.0 & 31.0 & 3.49 & 5.0 & 6.10 & 2.86 \\ 2.0 & 28.5 & 3.77 & \text { I0.0 } & 11.30 & 3.34\end{array}$

$\begin{array}{ccc}\frac{w_{\mathbf{H}}}{w_{0}}(\%) & H+h(\mathrm{~m} .) & H(\mathrm{~m} .) \\ 82 & & \\ 67 & 1.5 & 0.2 \\ 62 & 7.2 & 0.9 \\ 58 & 28.5 & 1.8 \\ & 28.9 & 3.5\end{array}$

The ice on the North-Siberian shelf varies in thickness between $2 \mathrm{~m}$ and $3.5 \mathrm{~m}$. Ice resistance and deflecting force must therefore play a more important role there than in the Weddell Sea. The waters on the North-Siberian shelf are characterized by a marked transition zone of great stability at a depth of 25 to $40 \mathrm{~m}$, separating an upper, lighter and practically homogeneous layer from a lower, heavier layer. The upper, homogeneous layer has, according to Sverdrup, a very high eddy-viscosity; he concludes that this water slides with the ice as a solid body over the lower, stable layer, there being practically no frictional stress in the transition zone. This would seem to require much smaller driftvelocities than those actually observed by Sverdrup. If the upper layer moves as a solid over a frictionless stable zone, we must have equilibrium between the wind stress on the surface of the ice and the deflecting force acting on this solidly moving layer of ice and water. Assuming the upper layer to have the depth $L$ one finds

$$
\tau_{a}=\bar{\rho} L f w
$$

where $\bar{\rho}$ is the average density of ice and water, and $w$ the common drift velocity. Setting $\rho=\mathrm{I}, f=\mathrm{I} .4 \cdot \mathrm{IO}^{-4}, L=2000 \mathrm{~cm}$., we find 


$$
w=3.57 \tau_{a}=\mathrm{I} 2.5 \cdot \mathrm{IO}^{-6} W_{0}^{2} ; \quad r=\frac{w_{0}}{W_{0}}=0.125 \cdot \mathrm{IO}^{-4} W_{0} .
$$

This value for the wind factor is far below the observed. If the effect of the ice resistance had been included, the computed wind factor would have been even less. To account for the observed values of the wind factor we must either assume that the stress is much greater than indicated by the Ekman-Taylor formula or we must assume that there is a great deal of shearing motion immediately below the ice. We have shown that aerological evidence speaks against the first alternative; we are forced to conclude that the ice slides over the water immediately below it. This sliding motion will necessarily produce a frictional force which, however, on account of the great internal ice resistance and consequent lower drift velocity, may be of minor importance. During the summer there is often a very shallow layer of fresher and lighter water next to the ice. In the meteorological section we have shown that shearing stresses are very effectively suppressed by a stable stratification. One should therefore expect that during the summer the ice drift on the North-Siberian shelf is controlled almost entirely by the wind force, the deflecting force and the internal ice resistance. This would apply particularly to light winds, since the increased stirring associated with higher winds and drifts undoubtedly tends to remove the shallow stable layer next to the ice and call into play frictional forces between drift ice and water, thereby again reducing the wind factor. During the winter season the shallow stable layer is missing. Because of this seasonal variation in hydrographic conditions and because of the large variation in the thickness of the ice and the ice resistance it seems reasonable to assume that the wind factor may obey different laws in winter and in summer.

If we disregard the effect of friction between ice and water $\left(\tau_{0}=0\right)$, the steady-state drift velocity of the ice may be computed from the balance between wind force $\left(\tau_{a}\right)$, internal ice resistance $(R)$ and deflecting force $(D)$. It is easily seen from Fig. $36 \mathrm{~b}$ that

$$
R \sin \varphi_{0}=D \cos \varphi_{0}
$$

and

$$
\tau_{a}=R \cos \varphi_{0}+D \sin \varphi_{0}
$$

or

$$
\tau_{a}=\frac{D}{\sin \varphi_{0}}
$$

Thus

and

$$
\rho \gamma^{2} W_{0}^{2}=\frac{M f w_{0}}{\sin \varphi_{0}}
$$

Thus the wind factor is proportional to the wind velocity and inversely proportional to the mass of the ice. To test this result and to verify our expression for the wind stress, we made use of the data in Table I of Sverdrup's paper. ${ }^{37}$ The wind factor was computed separately for the two winter seasons (Nov. I922-March 1923, Nov. I923-March 1924) 
and for two summer seasons (July-September I923 and July-August 1924). The results are given in Table 28 and in Fig. 40 and b. Turning first to the winter observations, it appears that the wind factor, in accordance with the theoretical prediction, is proportional to the wind velocity at all but light winds.

TABLE 28

Wind Factor as a Function of Wind Velocity (m.p.s.)

\begin{tabular}{|c|c|c|c|c|c|c|c|}
\hline & reriod & $1.0-1.9$ & $2.0-2.9$ & $3.0-3.9$ & $4.0-4.9$ & $5.0-6.9$ & $7.0-8.9$ \\
\hline \multirow{3}{*}{ Winter } & $\begin{array}{r}\text { Nov. Ig } \\
\text { March }\end{array}$ & $9^{\mathrm{I} .58}$ & ${ }_{16}^{2.49}$ & $3 \cdot 4416^{1.21}$ & $4 \cdot 345^{1 \cdot 5^{I}}$ & $5.879^{\mathrm{I} .6 \mathrm{I}}$ & $7.654^{2.05}$ \\
\hline & $\begin{array}{r}\text { Nov } \\
\mathrm{M} \\
\end{array}$ & 1.65 I0 1.85 & $2.37{ }_{\text {I9 }} \mathrm{I.23}$ & $3.505^{0.71}$ & $4 \cdot 349^{\text {I.55 }}$ & $5 \cdot 17 \cdot 3^{2.19}$ & $7.00,3.33$ \\
\hline & Bo & 1.621 .85 & $\begin{array}{r}2.43 \\
35 \\
\end{array}$ & $3.6_{21}^{1.08}$ & $4 \cdot 34{ }_{14}{ }^{\mathrm{I} \cdot 54}$ & $5.69 \mathrm{I} 2^{\mathrm{I}} .74$ & $7.5^{2} 5^{2 .}$ \\
\hline \multirow{3}{*}{ Summer } & $\begin{array}{r}\text { Jul } \\
\mathrm{I}\end{array}$ & I.38 $6^{\text {I.94 }}$ & $2.417^{2.65}$ & ${ }_{10}^{3.4 \mathrm{I}}{ }^{2.22}$ & $4.205^{2.19}$ & $5.684^{2.18}$ & $7.20 \mathrm{I}($ \\
\hline & $\begin{array}{r}\text { July } \\
\text { I }\end{array}$ & I. $445^{6.49}$ & $2.352^{3.77}$ & $3 \cdot 347^{4 \cdot 32}$ & $4.50 \quad(0.04)$ & $-{ }_{0}-$ & $7.70 \quad$ I \\
\hline & Bo & I.4I II $^{4.05}$ & $2.40 \quad 9^{2.89}$ & $3 \cdot 3^{8}{ }_{17}^{3.07}$ & $4.256^{\mathrm{I.8I}}$ & $5.684^{2.18}$ & $7 \cdot 45_{2}$ \\
\hline
\end{tabular}

In each box the upper left number represents wind velocity, the upper right number wind factor. The number of observations is stated at the bottom of each box.

Due to the forces acting between ice fields moving in different directions, the movement of the ice at the place of observation must be controlled to a certain extent by conditions prevailing at a distance. The small component of the ice drift due to winds and resistance forces in a distance will become noticeable when the local wind and drift are slight. To test this explanation, we have tabulated the distribution of the angle between surface drift and surface wind (counted positive cum sole). Table 29 shows that this distribution almost is of random nature for very light winds; thus there is practically no connection between the local light wind and the movement of the ice.

TABLE 29

Frequency Distribution of Angle of Surface Drift Measured cum sole from Surface Wind

\begin{tabular}{|c|c|c|c|c|c|c|c|}
\hline \multirow{5}{*}{ Winter } & Quadrant & $1.0-1.9$ & $2.0-2.9$ & $3.0-3 \cdot 9$ & $4.0-4.9$ & $5.0-6.9$ & $7.0-8.9$ \\
\hline & I & I I & $2 \mathrm{I}$ & I 5 & 13 & II & 5 \\
\hline & 2 & 0 & 4 & o & o & 0 & o \\
\hline & 3 & 3 & 0.5 & 2 & 0 & 0 & 0 \\
\hline & 4 & 5 & 9.5 & 4 & $\mathbf{I}$ & $\mathbf{I}$ & 0 \\
\hline \multirow{4}{*}{ Summer } & I & 7 & 7 & 12 & 5 & 4 & 2 \\
\hline & 2 & 2 & o & 4 & 0 & 0 & 0 \\
\hline & 3 & $\circ$ & I & I & o & $\circ$ & 0 \\
\hline & 4 & 2 & I & 0 & I & 0 & 0 \\
\hline
\end{tabular}

If we now insert in (I64) the numerical values $\rho \gamma^{2}=35 \cdot 10^{-7}, f=1.4^{\cdot} \cdot \mathrm{O}^{-4}, \varphi_{0}=30^{\circ}$, it follows that

$$
\frac{w_{0}}{W_{0}} \cdot \mathrm{IO}^{2}=\frac{\mathrm{I} .25}{M} W_{0}
$$




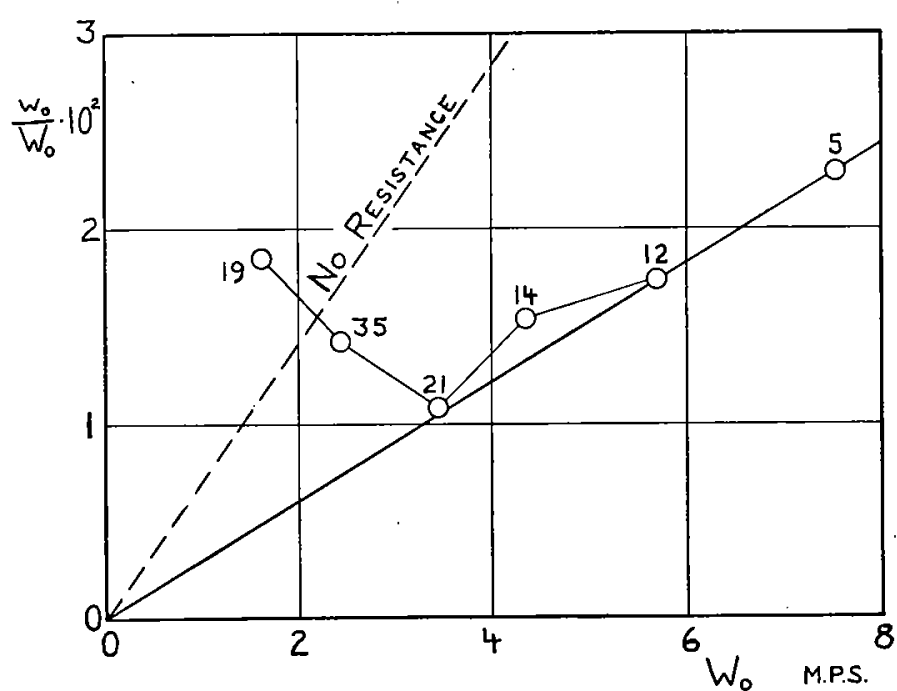

Fig. 40a.-Wind factors on the North-Siberian shelf plotted against

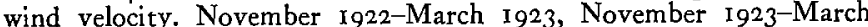
I924. Computed from Sverdrup's observations.
Fig. 4oa shows that for moderate and fresh winds

$$
\frac{w_{0}}{W_{0}} \cdot 10^{2}=0.003 W_{0}
$$

Thus

$$
M=4 \mathrm{I} 7 \mathrm{grams} / \mathrm{cm}^{2} .
$$

Actually, the thickness of the ice is about $3.5 \mathrm{~m}$ during the winter. If the density of the ice is taken to be 0.9 grams $/ \mathrm{cm}^{3}$, it follows that its mass is 315 grams $/ \mathrm{cm}^{2}$. Thus our analysis indicates that we may account for the drift of the arctic ice by Sverdrup's method of attack, provided we increase the actual mass of the ice by $32 \%$, to allow for the resistance between ice and water. The broken line in Fig. 4oa represents the maximum value of the wind factor in the absence of ice resistance and friction between ice and water. It is computed from ( $\mathrm{I} 64$ ) for $\varphi_{0}=90^{\circ}, M=350 \mathrm{grams} / \mathrm{cm} .^{2}$ and proves conclusively that the large wind factor obtained from the light wind group must be due to other causes than local winds.

During the late summer the wind factor at first decreases with increasing wind velocity and then appears to increase slightly as the latter exceeds 4 m.p.s. Table 29 shows that for winds lighter than 2 m.p.s. the direction of the drift vector bears practically no relation to the wind direction. We are therefore justified in disregarding the corresponding point in Fig. 4ob. The rapid decrease in the wind factor between 3 m.p.s. and 4 m.p.s. is at least in part due to the disappearance of the stable layer below the ice. If we assume that this stirring has not yet set in for a wind velocity of 3.4 m.p.s. we may use the corresponding point in the diagram to compute $M$ during the summer. Assuming $\rho \gamma^{2}=35 \cdot$ IO $^{-7}, W_{0}=3.4$ m.p.s., $r=3.1, \varphi_{0}=40$, we obtain

$$
M=\mathrm{I} 70 \mathrm{grams} / \mathrm{cm}^{2} .
$$

The thickness of the summer ice is about $2 \mathrm{~m}$,

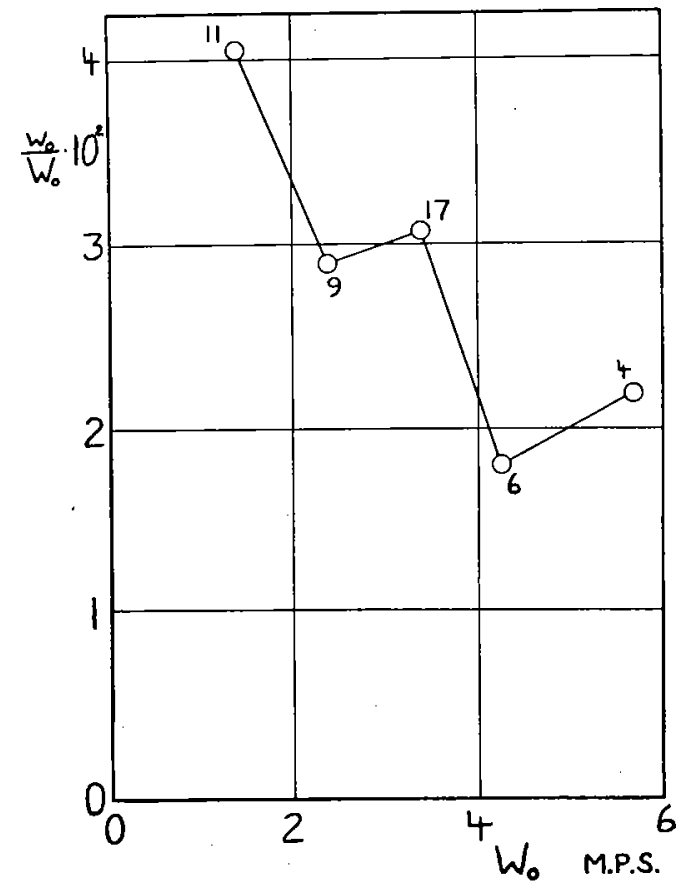

FIG. 4ob.-Wind factors on the North-Siberian shelf plotted against wind velocity. July-September I923, July-August I924. Computed from Sverdrup's obser- 
its average mass consequently 180 grams $/ \mathrm{cm} .{ }^{2}$. This agreement is satisfactory, verifying the assumption that for these light winds resistance between ice and water is insignificant.

In conclusion, it may be said the Brennecke's observations of the ice-drift in nearly all their details furnish a striking verification of the drift-current theory presented in (A) and developed further in the present paper. Sverdrup's observations of the ice-drift and his pilot balloon observations clearly demonstrate the general validity of the Ekman-Taylor formula for the wind stress.

\section{RELATION BETWEEN THE VELOCITY PROFILE AND THE VALUE OF THE MIXING LENGTH}

\section{Theoretical Comments}

It was shown in (A) that the maximum value of the mixing length in a homogeneous layer $(h)$ of frictional influence is given by

$$
l_{\max }=\frac{k h}{\sqrt{2}} \text {. }
$$

In the present paper evidence has been presented which indicates that $k$ has a value of about 0.065 , both in the atmosphere and in the ocean. Thus the ratio between the maximum mixing length and the depth of the layer of frictional influence (excluding the thin boundary layer) is given by

$$
\frac{l_{\max }}{h}=\frac{k}{\sqrt{2}}=\frac{0.065}{\sqrt{2}}
$$

It is interesting to note that Nikuradse ${ }^{40}$ on the basis of numerous measurements in pipe lines finds

$$
\frac{l_{\max }}{\operatorname{diam} .}=0.070
$$

as the limiting value for large Reynold's Numbers. Tollmien ${ }^{41}$ in a study of wake stream phenomena, assumes $l$ to be constant across the stream and obtains

$$
\frac{l}{\text { width }}=0.0682
$$

for the mixing zone between a homogeneous moving air current and quiet air (two dimensional case). In the case of mixing between a circular air jet and the surrounding resting air he finds

$$
\frac{l}{\operatorname{diam} .}=\frac{0.0729}{2}
$$

The expressions (168) and (170) differ by the factor $\frac{1}{2}$; this is easily understood if one assumes that the circular jet may be regarded as a conical wake stream, the width of which is equal to the radius of the wake stream.

These illustrations seem to point to the conclusion that the maximum value of the mixing length is of the form

$$
l=\frac{c d}{n}
$$


where $d$ is the diameter, or width, of the system and $c$ is a non-dimensional number approximately equal to 0.07 , but, according to Tollmien, decreasing slightly with increasing R.N. The number $n$ apparently depends upon the geometric character of the flow.

We shall not attempt to carry this suggestion further but wish to emphasize that the comparisons made above strengthen the confidence that may be placed in our previous determinations of $k$.

Von Kármán's principle of similarity on which most of our developments are based, was first applied by him to flow through pipe lines. An inspection of Nikuradse's data shows that the actual variation of the mixing length with the distance from the wall $(y)$ is fairly well represented by assuming Prandtl's law

$$
l=k_{0} y=0.4 y
$$

near the wall and

$$
l=0.07 d=0.14^{r}
$$

in the remainder of the channel. From the condition of continuity in $\mathrm{I}$ it follows that

$$
H=0.35 r
$$

where $H$ is the thickness of the boundary layer within which Prandtl's expression for l applies.*

The solution obtained from von Kármán's ${ }^{3}$ theory leads to a quite different distribution of $l$ with a maximum value at the distance $r / 4$ from the center. At the center of the pipe line $l=0$. The apparent agreement between von Kármán's theory and the observed velocity distribution is largely due to the fact that von Kármán plots $\left(u_{\max }-u\right) / \sqrt{\tau_{0} / \rho}$ against $\mathrm{I}-y / r$. This method of representation automatically insures complete agreement between theory and observations in the center part of the channel where the velocity profile is flat. In the case of rough pipe lines there is some disagreement between theory and observations in the regions near the wall.

It would seem that a necessary requirement for the application of von Kármán's principle must be that the velocity profile be geometrically similar to itself, i.e., it should be possible to make any finite portion of the velocity profile coincide with any other portion by changing velocity and length scales. This condition is not fulfilled in the case of the pipe line.

When the similarity principle is applied to pure drift currents $(A, I V)$ the resulting velocity distribution has the form of a logarithmic spiral. In the atmosphere, the vectorial difference between the actual wind and the constant gradient wind describes a logarithmic spiral. Since any portion of a logarithmic spiral may be made to coincide with any other portion by a simple rotation and a change in length scale it appears that conditions are more favorable for the application of von Kármán's principle of similarity to these geophysical phenomena than to flow in pipe lines.

If we demand that the mean motion be dynamically similar to itself the stress distribution must have the character of a logarithmic spiral. It can easily be shown that the vertical distribution of the eddy-viscosity then must obey one of the three following laws

$$
\eta=A(h-z)^{2}, \quad \eta=B, \quad \eta=C(z+b)^{2},
$$

* This distribution of the mixing length is now being studied by Dr. H. Peters and one of us (C.-G.R.) in an analysis of certain boundary layer problems. 
where $A, B$ and $C$ are independent of $z$. The first law includes the solutions given in (A) and in the present paper. The second case corresponds to the classical solution by Ekman and the third case to Solberg's solution. ${ }^{8}$

Included in the first alternative is the particular case of constant $l$, corresponding to the basic assumption of Tollmien's theory. This case was actually treated by Ekman in 1905, who determined the characteristics of a pure drift current in which the frictional force was proportional to the square of the shear.

The proof of the above theorem follows. The equations of motion are

$$
-\rho f i\left(U+i V-U_{o}\right)+\frac{\partial \tau_{x}}{\partial z}+i \frac{\partial \tau_{y}}{\partial z}=0 .
$$

The stress distribution follows a logarithmic spiral, thus

$$
\tau_{x}+i \tau_{y}=\tau_{s} e^{(\alpha+i) \psi}
$$

where $\psi$ represents the direction of the shear and $\tau_{s}$ is the value of the stress at the arbitrary level $\psi=0$. Differentiating equation (I76) with respect to $z$ one obtains

$$
-\rho f i C e^{i \psi}+\tau_{s}\left[(\alpha+i) \frac{d^{2} \psi}{d z^{2}}+(\alpha+i)^{2}\left(\frac{d \psi}{d z}\right)^{2}\right] e^{(\alpha+i) \psi}=0 .
$$

In this equation $C$, the rate of shear, is an unknown function of $z$ (or $\psi$ ). Separating real and imaginary parts one obtains

$$
\begin{aligned}
\frac{\rho f C}{\tau_{s}} e^{-\alpha \psi} & =\frac{d^{2} \psi}{d z^{2}}+2 \alpha\left(\frac{d \psi}{d z}\right) \\
\circ & =\alpha \frac{d^{2} \psi}{d z^{2}}+\left(\alpha^{2}-\mathrm{I}\right)\left(\frac{d \psi}{d z}\right)^{2} .
\end{aligned}
$$

From the second of these two equations it follows that

$$
\frac{d \psi}{d z}=A e^{\frac{1-\alpha^{2}}{\alpha} \psi}
$$

( $A$ is a constant)

and, after integration,

$$
e^{\frac{\alpha^{2}-1}{\alpha} \psi}=\frac{\alpha^{2}-I}{\alpha}(A z+B) .
$$

The rate of shear is given by (I79), which, with the aid of (I80) may be reduced to

$$
C=\frac{\tau_{s}}{\rho f} \frac{\mathrm{I}+\alpha^{2}}{\alpha}\left(\frac{d \psi}{d z}\right)^{2} e^{\alpha \psi}=\frac{\tau_{s}}{\rho f} \frac{\mathrm{I}+\alpha^{2}}{\alpha} e^{\frac{2-\alpha^{2}}{\alpha} \psi} .
$$

The eddy-viscosity is given by

$$
\eta=\frac{\tau_{8} e^{\alpha \psi}}{C}=\text { Constant } \cdot e^{2^{\frac{\alpha^{2}-1}{\alpha}} \psi}=\text { Constant } \cdot(A z+B)^{2} .
$$

Ekman's classical solution corresponds to

$$
\alpha^{2}=\mathrm{I}, \quad \frac{d \psi}{d z}=A
$$


If one introduces the assumption $d l / d z=0, l=$ constant $h$ (corresponding to $\alpha^{2}=4 / 3$ ), the resulting solution gives too large values of $h$ for all reasonable assumptions concerning the ratio $l / h$. Thus it appears that the original solution in $A$ describes conditions better than any other logarithmic spiral.

Our analysis may perhaps be summarized by saying that von Kármán's principle is applicable whenever the velocity profile has a marked curvature, while Tollmien's expression (I7I) for the mixing length holds when the velocity profile is quite flat. The velocity distribution in drift currents or in the frictional layer in the atmosphere is characterized by two regimes, one in which the rate of shear is strong and changing rapidly with depth (height), and another in which the vector of shear rotates rapidly with elevation (depth); the only point where the mixing length approaches Tollmien's value must be in the transition zone between these two regions.

There are nevertheless certain important types of motion, both in the ocean and in the atmosphere, where the vector of shear remains constant in direction and where the velocity profile is so flat that Tollmien's expression for the mixing length would seem appropriate. The tidal and permanent currents of the continental shelfs seem to belong to this group. An example will be given in the next section.

\section{Stirring in Shallow Water}

We have made use of a set of current measurements from the central part of Buzzards Bay, $41^{\circ} 30^{\prime} 40^{\prime \prime} \mathrm{N}, 70^{\circ} 49^{\prime} 30^{\prime \prime} \mathrm{W}$, in an attempt to apply Tollmien's method to a concrete geophysical example. These measurements were made on August 22, I934, between 0930 and 1630 , 60th meridian time. During this day the wind direction remained nearly constant (WSW) and approximately parallel to the axis of the Bay; the speed varied between 3 and 4 Beaufort (5-7.5 m.p.s.). The current was measured 3, 5, 7, 9, I I, $\mathrm{I} 3$, and $\mathrm{I} 5 \mathrm{~m}$ below the surface (bottom at about $\mathrm{I} 7 \mathrm{~m}$.). The measurements were then carried out in the reverse order and the entire procedure repeated a number of times.

The water temperature in the surface and at the bottom was measured twice. At I030 the surface temperature was $19.8^{\circ} \mathrm{C}$, the bottom temperature $19.5^{\circ} \mathrm{C}$. At 1600 the surface temperature was $20.0^{\circ} \mathrm{C}$, the bottom temperature $19.1^{\circ} \mathrm{C}$. The current observations indicate that the observed weak temperature gradient was concentrated in a layer close to the bottom. We can therefore assume that the main body of water was homogeneous.

By averaging successive measurements at the same level it was possible to obtain reliable synoptic velocity profiles. These profiles are represented graphically in Fig. 4I a and $b$. In each profile the current direction is constant from level to level, except at $\mathrm{I} 5 \mathrm{~m}$ where the readings are somewhat irregular. In the forenoon, the receding tide sets towards $100^{\circ}$ (magnetic direction). In the afternoon, the advancing tide sets towards $300^{\circ}$. The current has the character of a long, plane gravitational wave, along the axis of the bay, modified by a steady wind force applied at the surface. The effect of this shearing stress is very pronounced. In the forenoon, with the tide running against the wind, the velocity maximum normally to be found at, or just below the surface has been displaced downward to a depth of about I I $\mathrm{m}$. In the afternoon, when the tide runs with the wind, the velocity profile is characterized by a practically constant shear down to the vicinity of the bottom. 


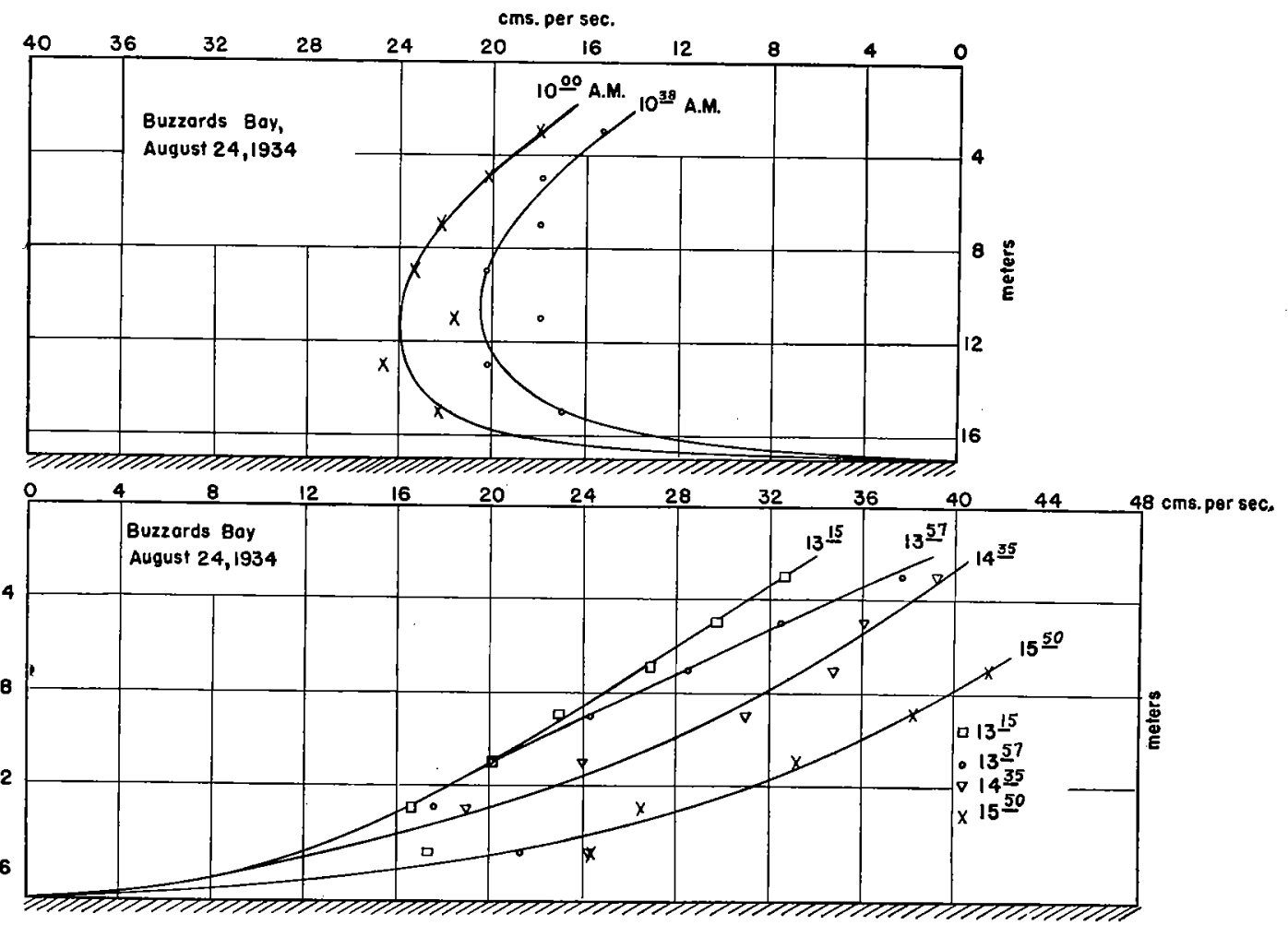

FIG. 4I a AND 4Ib.-Velocity profiles for tidal currents in the central part of Buzzards Bay on August 22, I934, showing the pronounced effect of the wind.

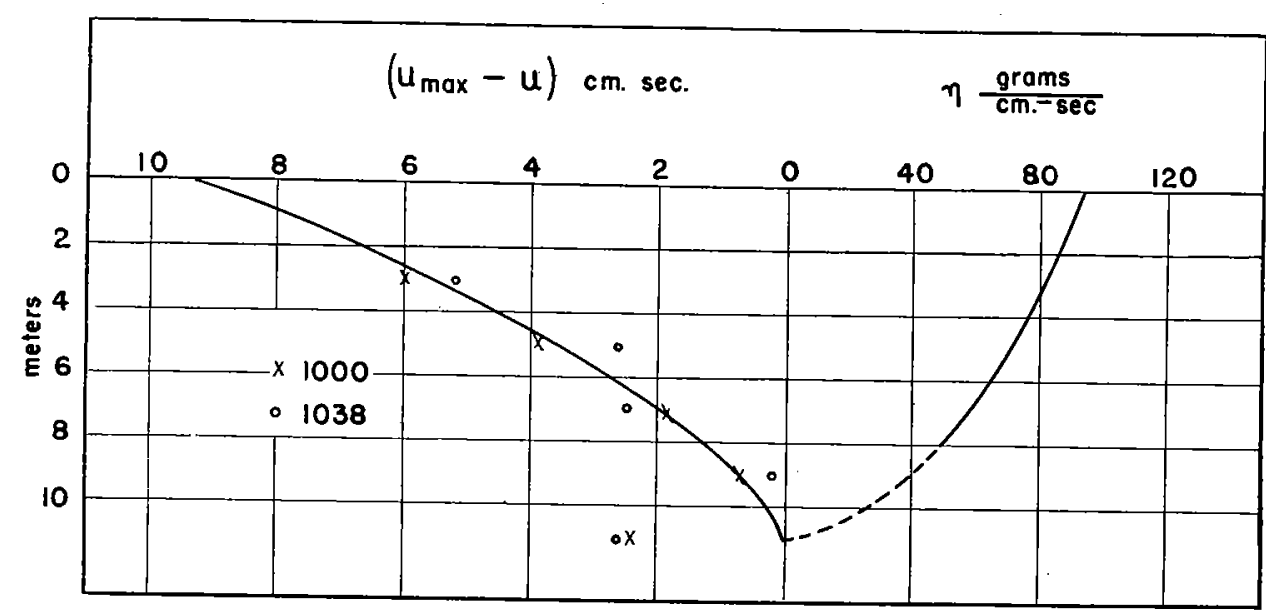

FIG. 42.-The left curve illustrates the theoretical retardation of the tidal curtent by an opposing wind. The rings and crosses indicate the observed retardation. The curve to the right gives the computed eddy-viscosity distribution. 
It follows from the wind stress formula that the wind force on the surface varies between 0.8 and 1.5 dynes $/ \mathrm{cm}^{2}$. We shall use a mean value of the stress,

$$
\tau_{0}=\text { I.2I dynes } / \mathrm{cm}^{2}, \quad \sqrt{\frac{\tau_{0}}{\rho_{w}}}=\text { I.I cm. } / \mathrm{sec} . \quad\left(\rho_{w}=\mathrm{I}\right) .
$$

The equation of motion for a long, gravitational wave is

$$
\rho_{w} \frac{\partial u}{\partial t}=-\rho_{w} g \frac{\partial \zeta}{\partial x}+\frac{\partial \tau_{z}}{\partial \xi} .
$$

In this equation $u$ is the velocity, $\zeta$ the vertical displacement of the surface from its equilibrium position and $\tau_{x}$ the shearing stress. In our case, this stress has a constant value $\left(\tau_{0}\right)$ at the surface. In the above equation the $z$-axis is counted positive upwards.

The two profiles obtained in the forenoon show that the decrease in current velocity between IIOO and II 38 is practically constant between the surface and I I $\mathrm{m}$. Since $\partial \zeta / \partial x$ is independent of $z$ it follows that $\partial \tau_{x} / \partial z$ is practically independent of $z$ within the first I I m. We know, at least approximately, the value of the shearing stress at the sur-
face. We also know that the shearing stress must vanish at a depth of about I I $\mathrm{m}$, where the shear $(\partial u / \partial z)$ vanishes. Consequently we know the value of the shearing stress at every depth between the surface and II $\mathrm{m}$.

If we assume that Prandtl's expression (3) for the shearing stress applies also to nonstationary motions, it follows that

$$
l^{2}\left(\frac{\partial u}{\partial z}\right)^{2}=\left|\frac{\tau}{\rho_{w}}\right|=\frac{\tau_{0}}{\rho_{w}} \cdot \frac{z}{h},
$$

where $z$ now is counted positive upwards from the I I $\mathrm{m}$ level and $h$ has the numerical value I roo.

The entire body of water is stirred both from below and from above, due to the action of tide and wind. The velocity profile is quite flat except near the bottom and at the I I $\mathrm{m}$ level. We shall therefore assume that the mixing length is constant throughout the upper i I m. It follows that

$$
\left|\frac{\partial u}{\partial z}\right|=\frac{\mathrm{I}}{l} \sqrt{\frac{\tau_{0}}{\rho_{w}}} \cdot \sqrt{\frac{z}{h}}
$$

and

$$
u_{\mathrm{max}}-u=\frac{2 h}{3 l} \sqrt{\frac{\tau_{0}}{\rho_{w}}} \cdot\left(\frac{z}{h}\right)^{3 / 2}
$$

Fig. 4 Ia indicates that $u_{\max }$ was $24 \mathrm{~cm} . / \mathrm{sec}$. at 1000 and $19.5 \mathrm{~cm} . / \mathrm{sec}$. at 1038 . In Fig. 42 we have plotted the observed velocity differences, $24.0-u$ and $19.5-u$, against $z$. In the same diagram we have drawn a curve

$$
u_{\max }-u=9 \cdot 3\left(\frac{z}{h}\right)^{3 / 2} .
$$


The constant 9.3 was chosen so as to make the curve fit the observations. It follows from (I89) and (I90) that

$$
\sqrt{\frac{\tau_{0}}{\rho_{w}}}=9 \cdot 3: \frac{3 l}{2 h}
$$

or,

$$
\frac{l}{d}=c=0.05 \mathrm{I}
$$

where $d$ represents the depth $(\mathrm{I} 7 \mathrm{~m})$. The value of $c$ is somewhat less than one should expect from Tollmien's theory. However, in view of the intermittent character of the tidal stirring the result is hardly surprising. The $3 / 2$-power law for the velocity distribution is nicely verified by the observations.

The eddy-viscosity distribution corresponding to this velocity profile has been computed from

$$
\eta=\rho_{w} l^{2}\left|\frac{\partial u}{\partial z}\right|=\rho_{w} l \sqrt{\frac{\tau_{0}}{\rho}} \cdot \sqrt{\frac{z}{h}} .
$$

The result is represented graphically by the curve to the right in Fig. 42 . We have indicated by a broken line the uncertain values in the vicinity of the level of disappearing shear.

We shall now attempt to apply these results to the afternoon data. The velocity profile for I 3 I 5 is very nearly a straight line, suggesting a constant stress. It is apparent that $\rho_{w} \frac{\partial u}{\partial t}$ and $-\rho_{w} \frac{\partial \zeta}{\partial x}$ must have the same sign. Thus, in the equation

$$
\frac{\partial \tau_{x}}{\partial z}=\rho_{w} \frac{\partial u}{\partial t}+\rho_{w} g \frac{\partial \zeta}{\partial x}
$$

the two terms on the right side will in part cancel each other. Since the wind speed at the time of this observation was somewhat greater than during the forenoon (4 Beaufort) it is reasonable to assume that the percentual variation of $\tau_{x}$ with depth must be small. We shall therefore assume

$$
\tau_{x}=\tau_{0}=\mathrm{I} .5 \text { dynes } / \mathrm{cm} .2, \sqrt{\frac{\tau_{0}}{\rho_{w}}}=\mathrm{I} .23 \mathrm{~cm} . / \mathrm{sec} .
$$

Between $3 \mathrm{~m}$. and $7 \mathrm{~m}$. the shear $(\partial u / \partial z)$ has the value $\mathrm{I} \cdot 4 \cdot 1 \mathrm{O}^{-2}$. It follows that

$$
l=\frac{\mathrm{I} 23}{\mathrm{I} \cdot 4}=88 \mathrm{~cm}
$$

Thus

$$
c=\frac{l}{d}=0.052
$$

This value agrees well with the one found above. 
The large shear in the curve for 1357 is undoubtedly due to a temporary increase in the wind speed. The upper part of the curve for 1435 has about the same slope as the curve for I 315 .

In conclusion, the data analyzed above seem to indicate that the intermittent stirring of shallow bodies of homogeneous water due to tides and wind produce values of the mixing length which approach but do not quite reach those obtained by Nikuradse and Tollmien.

\section{APPENDIX}

\section{Modified Computation of the Boundary Layer in Drift Currents}

In the chapter on drift currents in $A$, the solution has already been modified with the introduction of a surface layer where the mixing length increases linearly with depth. This was done in a manner parallel to that used in II, I for the atmosphere. The essential difference is that the amplitude of the surface roughness elements $(\epsilon)$ is fixed in a given locality for winds blowing over land, whereas in the open ocean it depends presumably only on the wind speed relative to the water. It was assumed that the value of the mixing length next to the surface $\left(l_{0}\right)$ was simply $\epsilon$, whereas for the atmosphere it is $k_{0} z_{0}=k_{0} s \epsilon$ where $s=\mathrm{I} / 30$ and hence $k_{0} s=.013$. It is probable that the corresponding quantity for open water $\left(k_{0} s_{w}\right)$ is more nearly unity, since $\epsilon$ represents the actual amplitude of the vertical displacements of the surface water, while the vertical displacements of the surface air may be cut way below $\epsilon$ due to the formation of dead pockets in the troughs between the roughness elements. We shall introduce the factor $k_{0} s_{w}$, and later (page 95) choose its value within the limits I and .oI3. This changes (AI2I) to

$$
\frac{\tau_{0}}{\rho_{w}}=k_{0}^{2}\left(z+s_{w} \epsilon\right)^{2}\left(\frac{d u}{d y}\right)^{2} .
$$

The first solution in A, derived on the assumption that the stress is parallel to the vector of shear, led to an angle between surface drift and wind stress of $\varphi_{0}=54^{\circ} 44^{\prime}$, which is greater than observations show. Accordingly a second solution was carried through, assuming a constant angle between stress and shear so as to make $\varphi_{0}=45^{\circ}$. But it should be pointed out that the values of $\varphi_{0}$ in Table Ar from the complete first solution, including the boundary layer, are smaller and agree with observed values within observational error. Hence it is quite unnecessary to make any other than the natural assumption that stress and shear are parallel.

In order to obtain numerical results from the solution in A, certain empirical relations were introduced, namely (AI09), (AIII) and (AI27). Since these are dimensionally incorrect, and since we have found the constant of the second to be wrong, we will make the following improvements.

The relation between surface wind speed and stress is given by

$$
\tau_{0}=\rho \gamma^{2} W_{0}^{2},
$$

in which expression $\gamma$ depends upon the height at which the wind is measured and upon the effective roughness of the sea surface. The actual roughness increases with increasing wind velocity. On the other hand, waves have a velocity nearly the same as the wind velocity and this would tend to reduce the effectiveness of the increasing roughness. 
Perhaps these two effects largely cancel. However, Witting ${ }^{42}$ has found that the wind storage of water in the Baltic is proportional to the wind. (The earlier relation of Colding gives it as proportional to the square of the wind). This is a very surprising result. In view of the indirect fashion in which Witting's result was derived as compared with the well established character of the general law (8), we have retained the proportionality between $\tau_{0}$ and $W_{0}^{2}$ in the subsequent development.

Introducing (8) in (A92), the depth of the layer of frictional influence, exclusive of the boundary layer, is

$$
h=3 \frac{k}{f} \sqrt{\frac{\tau_{0}}{\rho_{w}}}=3 \frac{\gamma k}{f} \sqrt{\frac{\rho}{\rho_{w}}} W_{0},
$$

where $\rho_{w}$ is the water density. The depth of the boundary layer is, from (Ar23),

$$
H=\frac{k h}{\sqrt{2 k_{0}}}-s_{w} \epsilon,
$$

and hence the total depth of the layer of frictional influence is

$$
h+H=3 \frac{\gamma k}{f} \sqrt{\frac{\rho}{\rho_{w}}}\left(\mathrm{I}+\frac{k}{\sqrt{2 k_{0}}}\right) W_{0}-s_{w} \epsilon .
$$

From $\left(A_{125}\right)$ we get for the angle $\left(\varphi_{0}\right)$ between wind stress and surface drift that

$$
\tan \varphi_{0}=\frac{2}{\sqrt{2}+3 Z}
$$

where

$$
Z=\frac{k}{k_{0}} \ln \left(\frac{3 k^{2}}{k_{0} s_{w} \epsilon f} \sqrt{\frac{\tau_{0}}{2 \rho_{w}}}\right)=\frac{k}{k_{0}} \ln \left(\frac{3 \gamma k^{2}}{k_{0} s_{w} \epsilon f} \sqrt{\frac{\rho}{2 \rho_{w}}} W_{0}\right) .
$$

The speed of the drift at depth $H$ is, from (A9o),

$$
w_{H}=\frac{\mathrm{I}}{k} \sqrt{\frac{2 \tau_{0}}{3 \rho_{w}}}=\frac{\gamma}{k} \sqrt{\frac{2 \rho}{3 \rho_{w}}} W_{0},
$$

and from (AI25) the speed of the surface drift is

$$
w_{0}=w_{H} \sqrt{\mathrm{I}+\sqrt{2} Z+\frac{3}{2} Z^{2}}=\frac{\gamma}{k} \sqrt{\frac{\rho}{\rho_{w}}\left(\frac{2}{3}+\frac{2 \sqrt{2}}{3} Z+Z^{2}\right)} W_{0} .
$$

$\epsilon$ was determined in A from Cornish's (43) empirical formula

$$
\epsilon=0.37 W_{0} \text {. }
$$

But a dimensional relation giving $\epsilon$ as a function of the wind speed, of the air and water densities, and of the acceleration of gravity must be of the form

$$
\epsilon \propto \frac{\rho W_{0}^{2}}{\left(\rho_{w}-\rho\right) g} .
$$


Using a non-dimensional constant $G$, this may be expressed as

$$
\epsilon=\frac{G}{g} W_{0}^{2} .
$$

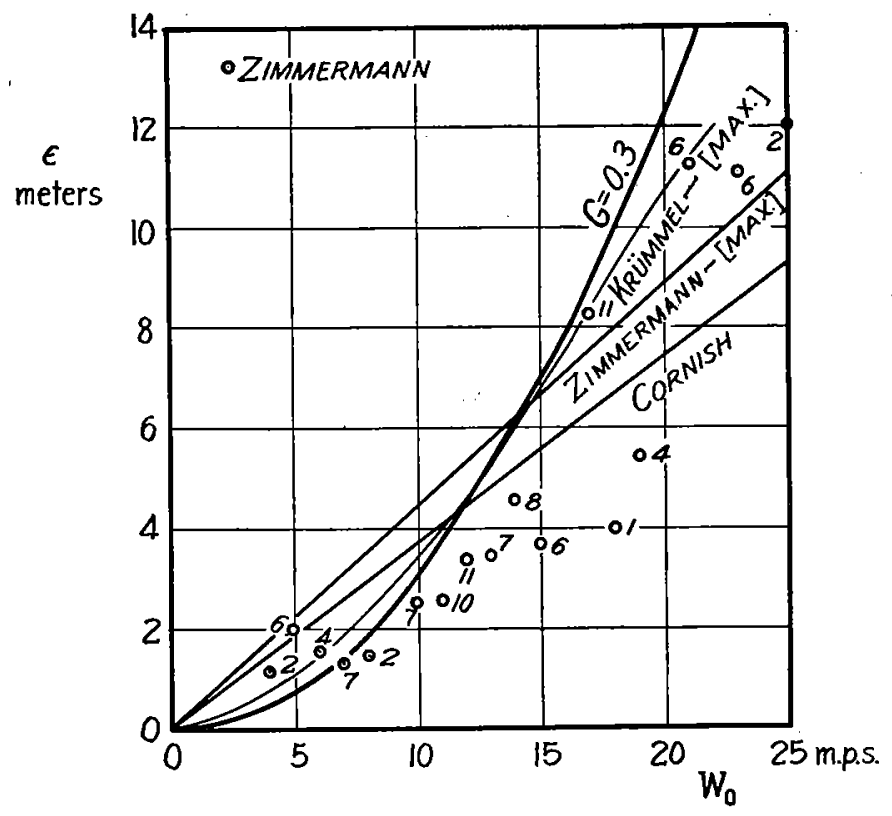

FIG. 43. The height of ocean waves is plotted against surface wind. The curve marked $G=0.3$ shows wave height proportional to the square of the wind velocity.
There is some observational justification for a quadratic relation instead of Cornish's linear one, for light and moderate winds. Krümmel, ${ }^{44}$ after a study of the various determinations of $\epsilon$, decides on values of the maximum amplitude of waves which agree more closely with the quadratic relation than with the linear one, as far as $W_{0}=20$ m.p.s. We are not concerned with winds stronger than this, since they are of too short duration to produce a steady state within the frictional layer, so we are justified in using (202), setting $G=0.3$. In Fig. 43 are plotted curve (202), Krümmel's curve, and Cornish's formula, as well as Zimmermann's ${ }^{45}$ more recent formula for the maximum height of waves:

$$
\epsilon=.44 W_{0}
$$

The points plotted in this figure are averages of measurements of $\epsilon$ made by.Zimmermann, the number of observations being shown beside each point.

Introducing (202) in (195), (196), (198), we obtain the following equations:

$$
\begin{aligned}
H & =\frac{k h}{\sqrt{2} k_{0}}-\frac{G s_{w}}{g} W_{0}^{2} \\
h+H & =3 \frac{\gamma k}{f} \sqrt{\frac{\rho}{\rho_{w}}}\left(\mathrm{I}+\frac{k}{\sqrt{2 k_{0}}}\right) W_{0}-\frac{G s_{w}}{g} W_{0}^{2} \\
Z & =\frac{k}{k_{0}} \ln \left(\frac{3 \gamma g k^{2}}{G k_{0} s_{w} f W_{0}} \sqrt{\frac{\rho}{2 \rho_{w}}}\right) .
\end{aligned}
$$

In equations (197), (200), (205) the first term gives the simple solution for the layer $h$, the succeeding ones are correction terms due to the inclusion of the boundary layer $H$. At very high winds, the sign of the sum of the correction terms changes, meaning that $H$ vanishes, so these terms should have to be neglected.

Although we have made allowance for the change of roughness with wind speed in its effect on the surface value of the mixing length in the water, we have neglected its effect 
on the value of the mixing length in the air at the sea surface, i.e., we have assumed a constant value of the stress factor $\gamma^{2}$ instead of a variable one given by (9). This seems at first to be inconsistent. But the assumption is probably very nearly correct, since the velocity of the water waves is nearly the same as the surface wind velocity, as has been pointed out on page $9^{2}$.

The value of 0.065 now established for $k$ makes the first term less than the second except at low latitudes in (AI3I), and has a similar effect on our new relation

$$
\frac{H}{h}=\frac{k}{\sqrt{2} k_{0}}-\frac{G s_{w f} f}{3 \gamma g k} \sqrt{\frac{\rho_{w}}{\rho}} W_{0},
$$

from (194) and (204), unless $k_{0} s_{w}$ is less than unity. If we continue to assume $k_{0} s_{w}=\mathrm{I}$, then the boundary layer does not exist according to (207) except at low winds and low latitudes. But this boundary layer serves a very useful purpose in reducing the angle between surface drift and wind stress, as mentioned above. Accordingly we will use a value of $k_{0} s_{w}$ that makes $H$ existent under all ordinary conditions, namely, $k_{0} s_{w}=\mathrm{r} / 6$.

These changes we have introduced necessitate a recomputation of Table AI. $h+H$ as computed from (205) is plotted in Fig. 29 , and $\varphi_{0}, w_{0} / W_{0}$ (the wind factor), $H / h$ as computed from (I97), (200), (206), (207) are shown in Tables 3I, 32, 33. $\varphi_{0}, H / h$ and $w_{0} / W_{0}$ are now functions of $W_{0}$ as well as of $f$, due to the introduction of the quadratic relation (202). The constants used in the calculations are as follows:

TABLE 30

$$
\begin{aligned}
\text { NoN-DIMENSIONAL } \\
\gamma=.05 \\
k=.065 \\
k_{0}=.38 \\
G=.3 \\
s_{w}=.44
\end{aligned}
$$

\begin{tabular}{|c|c|c|c|c|}
\hline \multirow[t]{2}{*}{$\mathrm{L}$} & \multicolumn{4}{|c|}{$W_{0}$ IN m.p.s. } \\
\hline & 5 & 10 & 15 & 20 \\
\hline $\begin{array}{l}15^{\circ} \\
30^{\circ} \\
45^{\circ} \\
60^{\circ} \\
75^{\circ} \\
90^{\circ}\end{array}$ & $\begin{array}{l}35.0^{\circ} \\
38.6^{\circ} \\
40.6^{\circ} \\
42.0^{\circ} \\
42.6^{\circ} \\
4^{2} .8^{\circ}\end{array}$ & $\begin{array}{l}38.7^{\circ} \\
42.8^{\circ} \\
45.4^{\circ} \\
46.8^{\circ} \\
47.7^{\circ} \\
48.0^{\circ}\end{array}$ & $\begin{array}{l}4 \mathrm{I} \cdot \mathrm{I}^{\circ} \\
45.7^{\circ} \\
4^{\circ} \cdot 4^{\circ} \\
50.2^{\circ} \\
51.1^{\circ} \\
51.4^{\circ}\end{array}$ & $\begin{array}{l}43.0^{\circ} \\
38.0^{\circ} \\
50.9^{\circ} \\
52.7^{\circ} \\
53.8^{\circ} \\
54.1^{\circ}\end{array}$ \\
\hline
\end{tabular}

\begin{tabular}{|c|c|c|c|c|}
\hline \multirow[t]{2}{*}{$\mathbf{L}$} & \multicolumn{4}{|c|}{$W_{0}$ IN m.p.s. } \\
\hline & 5 & IO & I 5 & 20 \\
\hline $15^{\circ}$ & .0317 & $.029 \mathrm{I}$ & .0276 & .0266 \\
\hline $30^{\circ}$ & .0292 & .0268 & .0254 & .0245 \\
\hline $45^{\circ}$ & .0280 & .0256 & .0243 & .0234 \\
\hline $60^{\circ}$ & .0273 & .0249 & .0237 & .0228 \\
\hline $75^{\circ}$ & .0269 & .0246 & .0246 & .0226 \\
\hline $90^{\circ}$ & .0268 & .0245 & .0233 & .0225 \\
\hline
\end{tabular}

TABLE 31

TABLE 32

$$
\frac{w_{0}}{W_{0}}
$$


TABLE 33

$\frac{H}{h}$

\begin{tabular}{c|cccc}
\hline L & & & $W_{0}$ IN m.p.s. & \\
& & 5 & 10 & I5 \\
\hline $15^{\circ}$ & .114 & .106 & .099 & .092 \\
$30^{\circ}$ & .107 & .093 & .079 & .063 \\
$45^{\circ}$ & .081 & .061 & .041 \\
$60^{\circ}$ & .096 & .072 & .047 & .023 \\
$75^{\circ}$ & .094 & .066 & .039 & .012 \\
$90^{\circ}$ & .093 & .064 & .036 & .008 \\
\hline
\end{tabular}

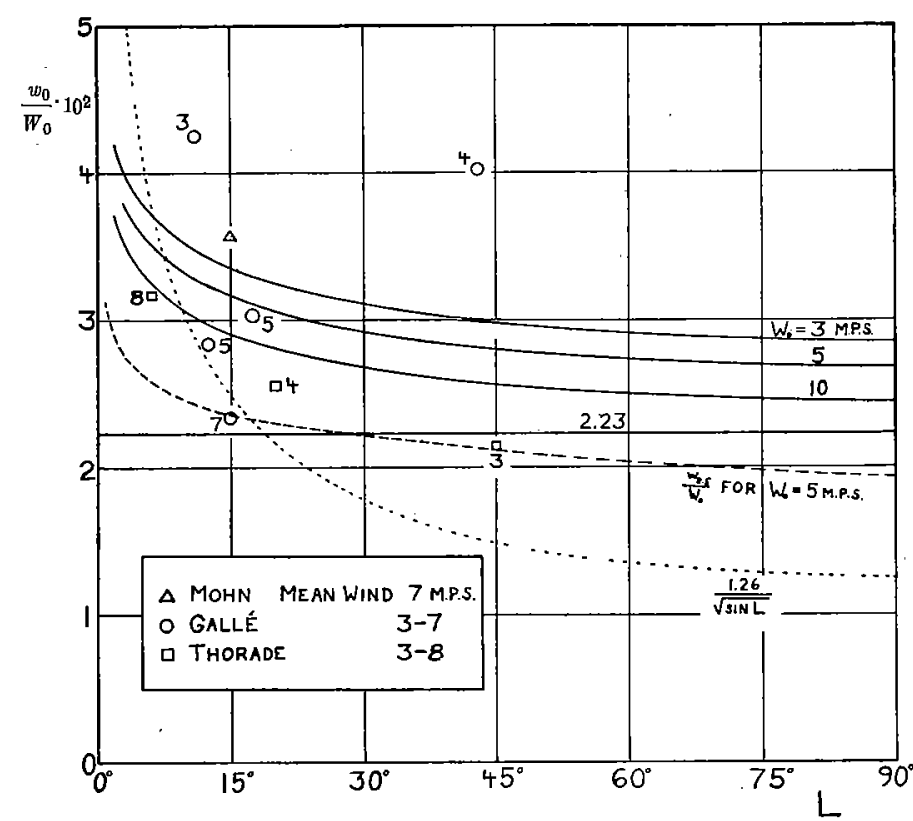

FIG. 44. The wind factor plotted against latitude, for the surface winds 3,5 , IO m.p.s. $2.23 \cdot 10^{-2}$ is the limiting value of the wind factor when the boundary layer vanishes. The number beside the observed values gives the mean wind speed.

$\begin{array}{ccc}\text { Mohn } & \text { Nansen } & \text { Dinklage } \\ .0103 & .0127 & .0127\end{array}$

In order to compare our theoretical values of the wind factor with the available observed ones, we have prepared Fig. 44, where wind factor is plotted against latitude. The full lines give the theoretical relation, lines being drawn for $W_{0}=3,5$, Io m.p.s. The horizontal line showing $w_{0} / W_{0}=.0223$ gives the limiting value of the wind factor at high winds when the boundary layer vanishes.

The dotted line represents Ekman's heretofore generally accepted $^{46}$ relation for the wind factor, namely

$$
\frac{w_{0}}{W_{.0}}=\frac{0.0126}{\sqrt{\sin L}} .
$$

The faith placed in this relation is largely based on the excellent agreement of the following determinations of the constant:

Witting Thorade 0.126

The agreement, however, is largely fortuitous. Nansen's value was determined over ice, where the wind factor may vary over a large range depending on the conditions, as is evident from IV, 7. Dinklage's and Witting's determinations were made in the Baltic Sea region, where the shallow water and coastal influences give conditions entirely different from the open ocean (see V). Furthermore Dinklage's measurements were made at a depth of 5 meters (and in only 12 meters of water).

In the same figure are plotted values taken from the papers of Mohn ${ }^{47} \mathrm{Galle}^{48}$ and Thorade. ${ }^{31}$ These determinations were made for the open ocean from the difference between the astronomical and dead reckoning positions of ships. Mohn used only cases where wind and surface current coincided in direction, which condition has turned out 
to be entirely at fault. Thorade attempted to correct this error by applying a factor $\mathrm{I} / \sqrt{2}$ derived from an analogy with coastal currents, obtaining the constant given under Mohn above. Unfortunately this analogy is quite imperfect. Accordingly we have plotted a value obtained from Mohn's means for the region $10^{\circ}-20^{\circ}$ north latitude. We have plotted mean values from Gallé for a number of different regions, but have not limited ourselves to his Kreislauf determinations, nor have we followed his example of dividing the current by the wind component in the direction of the current to obtain the wind factor. All of the plotted points have been reduced to the Beaufort scale of the Meteorological Office ${ }^{34}$ (Table 25). We have no reason for preferring this system to that of the Deutsche Seewarte, but use it merely to conform with IV, 4.

Although the plotted points agree well in order of magnitude with the theory, their scattering is large and indicates uncertainty in the determinations. Mohn's surface currents, due to his false assumption, must necessarily consist in part of permanent or "deep" currents. As mentioned by Thorade, Gallé's values must be considerably affected by extraneous currents too. Thorade's observations are the only ones from which the permanent currents have been eliminated. Unfortunately the great scattering in his seasonal means prevent too much reliance being placed on his values alone.

When the determinations are based on the drift of ships it is obvious that they do not refer to surface velocities, but rather to the velocities at some depth nearly a half of the draft of the ships. Although this has not been taken into account by any of the above mentioned authors, it is worthy of consideration due to the rapid shear in the surface layers. This rapid shear is illustrated by the velocity profiles in Fig. 4I $^{\mathrm{I}}$. Assuming the "drift center" of the average ship on which the wind factor determinations are based to be about 2.5 meters below water we have computed $w_{2.5} / W_{0}$ for $W_{0}=5 \mathrm{~m}$.p.s. and plotted it as the broken line in Fig. 44. This computation involves determining $h$ and $H$, then $W_{2.5}$ is found from the logarithmic formula (AI22a) if $H>2.5$ meters, otherwise it is found most easily from the velocity spiral for $\varphi_{s}=54^{\circ} 44^{\prime}$ in Fig. AI ( $w_{H} / W_{0}$ being always $.0223)$.

It is seen that Thorade's values fall reasonably well on such a family of curves for $w_{2.5} / W_{0}$. It must however be concẹded that Thorade's values according to the Beaufort scale he uses lie very well on the Ekman curve except for the low latitude value. But on the basis of all the evidence in favor of our general theory, it may be stated that Ekman's rapid variation of the wind factor with latitude is quite improbable. The only uncertainty about the theoretical variation with latitude rests in the thickness of the boundary layer (depending on the choice of $s_{w}$ ). The boundary layer cannot greatly exceed the one we have here assumed without giving too small values for $\varphi_{0}$, if it vanishes entirely it gives a constant wind factor $(=.0223)$.

The values in Table $3 \mathrm{I}$ for the angle between surface wind and surface drift are smaller than those for instance given by Defant. ${ }^{46}$ But these, again, are determined from the drift of ships and the change in the current direction is rapid with depth. As an example, the theory shows that for $L=30^{\circ}$ and $W_{0}=5$ m.p.s., $\varphi_{0}=39^{\circ}$ while $\varphi_{H=2.5}=55^{\circ}$. For an actual illustration it may be mentioned that Brennecke ${ }^{38}$ finds that the current direction changes $19^{\circ}$ in the first two meters below the ice, though of course the shear could not be so rapid as this in open water. 


\section{SUMMARY}

This paper may be regarded as a completion of and check on certain theoretical studies published by one of us in 1932. With the aid of conceptions first introduced by the Göttingen school of aerodynamics and adapted to geophysical problems in the abovementioned paper we have attempted to express the characteristic properties of the atmospheric and oceanic turbulent layers in terms of their controlling parameters and in a form which permits practical utilization of the results.

The theory leads to the introduction of a controlling non-dimensional number $N$, defined by $N=U_{o} / f z_{0}$, in which expression $U_{g}$ represents the gradient wind velocity, $t=2 \Omega \sin L$ the local angular velocity of the earth and $z_{0}$ a length characteristic of the roughness of the ground at the place of observation. We have determined the roughness parameter $z_{0}$ for different surfaces from the observed vertical variation in the wind velocity next to the ground and from other considerations.

The angle $\varphi_{s}$ between surface wind and gradient wind depends, in the case of an adiabatic atmosphere, on $N$ only and decreases slowly with increasing $N$. This result is clearly supported by available observations. Deviations at low wind velocities may be satisfactorily explained if one takes into account the effect of residual turbulence at higher levels.

The theory indicates that the thickness of the atmospheric boundary layer is proportional to the gradient wind velocity and to $\sin \varphi_{s}$; thus it increases with increasing roughness. It is inversely proportional to the Coriolis' parameter $f$. The two first conclusions are verified by a careful analysis of the observed depths of the turbulent layer over the aerological station in Boston.

The theory confirms the proportionality between surface stress and the square of the surface wind velocity obtained earlier by G. I. Taylor. A new method of determining the surface stress is discussed and numerical examples are given.

The effect of thermal stability on the vertical variation of the wind is analyzed. The theory indicates that the velocity distribution varies between the logarithmic type in the adiabatic case and a power law $(W \propto \sqrt{z})$ in the extremely stable case. This is confirmed by an analysis of Hellmann's wind data from Nauen. Heywood's observations furnish a thorough check on the theoretical relation between temperature gradient and wind gradient.

Thermal stability increases the angle between surface wind and gradient wind. The increase depends upon the non-dimensional parameter $g \frac{d \theta}{d z} / \theta f^{2}$, where $\theta$ is the potential temperature and $g$ is the acceleration of gravity. For high values of the stability a limiting angle of about $39^{\circ}$ is reached. This result is verified by Sverdrup's data from the Arctic and by observations contained in the Cardington report on wind structure.

The wind velocity very close to the ground $(z<5 \mathrm{~m}$.) varies with the shearing stress but does not to any marked degree depend upon the thermal stratification. The theory gives a definite relation between shearing stress at the ground and stability within the layer of frictional influence. This relation is verified by Hellmann's data for the diurnal variation of the wind velocity $2 \mathrm{~m}$ above the ground.

The introduction of a slight amount of thermal stability seems to cause profound changes in the characteristics of an originally adiabatic boundary layer but further increase in the stability produces relatively slight additional adjustments. 
The theory leads to definite conclusions regarding the structure of pure drift currents. These results are supported by an analysis of the observed depths of the homogeneous surface layer and by a study of the wind factor (ratio between surface drift and surface wind velocity).

Careful analysis of vertical temperature profiles obtained at selected hydrographic stations shows that the depth of the homogeneous layer is proportional to the surface wind speed. There is also a definite indication of an inverse proportionality to $\sin L$. Both these results are in agreement with the theory.

Additional data for this investigation have been obtained with the aid of a new type of sea water baro-thermograph, which records temperature directly against pressure.

In view of the difficulty of obtaining reliable values for the surface drift in the open ocean particular attention has been paid to the wind drift of the sea ice in Arctic and Antarctic waters. Brennecke's data on the ice drift and on the current velocities below the ice check the theory in every important respect and his data as well as Sverdrup's affirm the general validity of the Taylor formula for the wind stress.

Earlier determinations of the wind factor for open water are critically examined in an appendix to the present paper.

In the last part of the paper we have attempted to analyze the relation between the value of the mixing length and the curvature of the velocity profile. In case of very flat velocity profiles, it appears that the value of the mixing length may be estimated with the aid of a law deducible from Tollmien's results regarding wake stream phenomena. In this connection we have determined the value of the mixing length for a particular case of tidal stirring in shallow, nearly homogeneous water and obtained values which approach but do not fully reach those predicted by the Tollmien formula.

The entire theoretical development has been carried out on a dimensionally correct basis and with the aid of only three fundamental non-dimensional constants. One of these $\left(k_{0}\right)$ has already been determined by Prandtl and another $(k)$ appears to be identical with a constant first introduced by Tollmien.

\section{REFERENCES}

I. H. Ertel, I932: Allgemeine Theorie der Turbulenzreibung und des "Austausches," Sitzungsberichte der Preussischen Akademie der Wissenschaften, Phys.-Math. Klasse, XXVI.

2. C.-G. Rossby, I932: A Generalization of the Theory of the Mixing Length with Applications to Atmospheric and Oceanic Turbulence, Massachusetts Institute of Technology Meteorological Papers, Vol. I, No. 4.

3. Th. von Kármán, I930: Mechanische Ähnlichkeit und Turbulenz, Nachrichten von der Gesellschaft der Wissenschaften zu Göttingen, Math.-Phys. Klasse, Heft I, p. 58.

4. V. W. Ekman, I932: Studien zur Dynamik der Meeresströmungen, Gerlands Beiträge, Band 36, p. 385 .

5. L. Prandtl, I932: Meteorologische Anwendung der Strömungslehre, Beiträge zur Physik der freien Atmosphäre, Bjerknes-Festschrift, p. I88.

6. G. I. Taylor, I9I6: Skin Friction on the Earth's Surface, Proceedings of the Royal Society of London, A, Vol. 92, No. A637. 
7. P. Mildner, 1932: Über die Reibung einer speziellen Luftmasse in dem untersten Schichte der Atmosphäre, Beiträge zur Physik der freien Atmosphäre, Band 19.

8. H. Solberg, 1923: Sur le frottement dans les couches basses de l'atmosphère, Forh. Skand. Naturforskarmotet i Goteborg, Vol. 57, p. 95.

9. G. Hellmann, I917: Über die Bewegung der Luft in den untersten Schichten der Atmosphäre, Zweite Mitteilung, Sitzungsberichte der königlich Preussichen Akademie der Wissenschaften, p. I74.

I0. G. Hellmann, I9 15: Über die Bewegung der Luft in den untersten Schichten der Atmosphäre, Meteorologische Zeitschrift, Band 32, p. I.

I I. G. Hellmann, I919: Über die Bewegung der Luft in den untersten Schichten der Atmosphäre, Dritte Mitteilung, Sitzungsberichte der königlich Prèussisschen Akademie der Wissenschaften, p. 404.

I2. N. Shaw, I93 I: Manual of Meteorology, Cambridge University Press, Vol. 4, p. I23.

I3. G. Wüst, I920: Die Verdunstung auf dem Meere, Veröffentlichungen des Instituts für Meereskunde an der Universität Berlin, Neue Folge A, Heft 6, p. 73.

I4. E. H. Chapman, I9I9: Variation of Wind Velocity with Height, Meteorological Office Professional Notes, No. 6.

1 5. Aus dem Archiv der Deutschen Seewarte, a number of reports beginning with Band 40, Nr. 4, I922.

I6. V. W. Ekman, I927: Eddy-Viscosity and Skin-Friction in the Dynamics of Winds and Ocean-Currents, Memoirs of the Royal Meteorological Society, Vol. 2, No. 20.

17. H. Jeffreys, I920: On the Relation between Wind and Distribution of Pressure, Proceedings of the Royal Society of London, A, Vol. 96, p. 233.

I 8. G. M. B. Dobson, 1914: Pilot Balloon Ascents at the Central Flying School, Upavon, during the Year I9I3, 2uarterly Fournal of the Royal Meteorological Society, Vol. 40, p. I23.

I9. J. Hann und R. Süring, I926: Lehrbuch der Meteorologie, Leipzig, 4th ed., p. 5 I9.

20. E. van Everdingen, I909: Über die Ermittlung des Winkels zwischen Gradient und Windrichtung, Meteorologische Zeitschrift, Vol. 26, p. 75.

21. K. O. Lange and C. S. Draper, 1934: The Meteorological Airplane Ascents of the Massachusetts Institute of Technology, Papers in Physical Oceanography and Meteorology, Vol. 3, No. 2.

22. W. R. Gregg, L. T. Samuels and W. R. Stevens, I93I: The Meteorology of the Free Atmosphere, Bulletin of the National Research Council, No. 79, p. 89.

23. H. C. Willett, 1933: American Air Mass Properties, Papers in Physical Oceanography and Meteorology, Vol. 2, No. 2, p. I6.

24. F. M. Exner, 1927: Abhängigkeit des Turbulenzfactors der Winde von der vertikalen Temperaturverteilung, Sitzungsberichte der Akademie Wien, Math.-Naturwiss. Klasse, Abt. IIa, Band I36, p. 453 .

25. H. Ertel, I932: Die Abhängigkeit des Turbulenzkoeffizienten von der vertikalen Temperaturverteilung, Gerlands Beiträge, Band 35, p. $29 \mathrm{I}$.

26. G. S. P. Heywood, I93I: Wind Structure Near the Ground and its Relation to Temperature Gradient, Quarterly Fournal of the Royal Meteorological Society, Vol. 57, p. 433 .

27. M. A. Giblett, I932: The Structure of Wind over Level Country, Geophysical Memoirs, Meteorological Office, No. 54.

28. H. Hergesell, I922: Der tägliche Gang der Temperatur in der freien Atmosphäre 
über Lindenberg, Die Arbeiten des Preussischen Aeronautischen Observatoriums bei Lindenberg, Band I4.

29. H. U. Sverdrup, r933: Meteorology, Part I, Discussion, The Norwegian North Polar Expedition with the "Maud" I9I8-I925, Scientific Results, Vol. 2.

30. V. W. Ekman, I905: On the Influence of the Earth's Rotation on Ocean Currents, Arkiv för Matematik, Astronomi och Fysik, Band 2, No. I1, p. 39.

3r. H. Thorade, I914: Die Geschwindigkeit von Triftströmungen und die Ekmansche Theorie, Annalen der Hydrographie und Maritimen Meteorologie, Band 42, p. 379.

32. Discovery Reports, Falkland Islands, Vol. I, I929, p. I ; Vol. 3, I930, p. I.

33. Some of the Atlantis observations are published in Bulletin Hydrographique pour l'Annee 1932 and in H. B. Bigelow, I933: Studies of the Waters on the Continental Shelf, Cape Cod to Chesapeake Bay, Papers in Physical Oceanography and Meteorology, Vol. 2, No. 4.

34. G. C. Simpson, I926: The Velocity Equivalents of the Beaufort Scale, Meteorological Office Professional Notes, No. 44 .

35. See, for instance, H. L. Rietz and col., I 927: Handbook of Mathematical Statistics, Houghton Mifflin, p. 77.

36. H. U. Sverdrup, I 928 : Die Eistrift im Weddellmeer, Annalen der Hydrographie und Maritimen Meteorologie, Band 56, Heft 9, p. 265.

37. H. U. Sverdrup, I928: The Wind-Drift of the Ice on the North-Siberian Shelf, The Norwegian North Polar Expedition with the "Maud" I 918-I 925, Scientific Results, Vol. 4 , No. I.

38. W. Brennecke, I92 I : Die ozeanographischen Arbeiten der Deutschen Antarktischen Expedition I9II-I9I2, Aus'dem Archiv der Deutschen Seewarte, Band 34, No. I, p. 195 .

39. H. U. Sverdrup, 1930: Meteorology, Part II, Tables, The Norwegian North Polar Expedition with the "Maud" I9I 8-I925, Scientific Results, Vol. 3, Part II, p. 223.

40. J. Nikuradse, I933: Strömungsgesetze in rauhen Röhren, Verein Deutscher Ingenieure, Forschungsheft Nr. 36r.

$4^{\mathrm{T}}$. W. Tollmien, I926: Berechnung turbulenter Ausbreitungsvorgänge, Zeitschrift für angewandte Mathematik und Mechanik, Band 6, p. 468.

42. R. Witting, I909: Zur Kenntnis des vom Winde erzeugten Oberflächenströmes, Annalen der Hydrographie und Maritimen Meteorologie, Band 37, p. I93.

43. V. Cornish, Ig09: Wind-waves in Water, Sand and Snow, Nature, Vol. 80, p. I Ig.

44. O. Krümmel, I9II: Handbuch der Ozeanographie, Stuttgart, 2nd ed., Band.2, p. 74 .

45. E. Zimmermann, I920: Aufsuchung von Mittelwerten für die Formen Ausgewachsener Meereswellen auf Grund alter und neuer Beobachtungen, Schiffbau, Band 2I, p. 633 .

46. A. Defant, I929: Dynamische Ozeanographie, Einführung in die Geophysik III, Berlin, p. 68.

47. H. Mohn, I883: The North Ocean, Its Depths, Temperature and Circulation; The Norwegian North Atlantic Expedition 1876-1878, Christiania, Vol. 2, Part 2, p. II7.

48. P. H. Gallé, IgIo: Zur Kenntnis der Meeresströmungen, Mededeelingen en Verhandelingen, Utrecht, No. 9. 